Dynamics and characteristics of black aurora as observed by high resolution ground-based imagers and radar

Jenny Archer

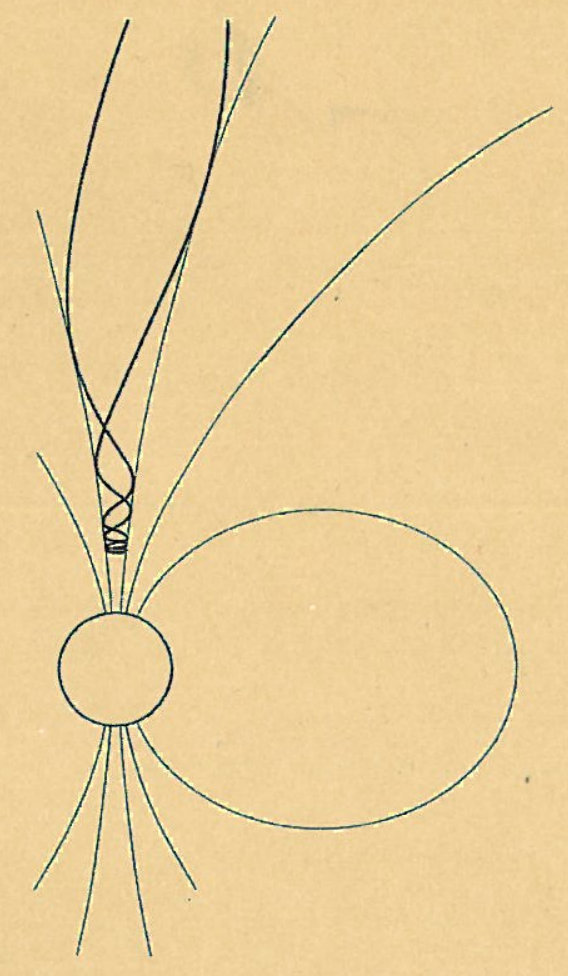

Master of Science Thesis

Stockholm, Sweden 2009 
KTH Electrical Engineering

\title{
Dynamics and characteristics of black aurora as observed by high resolution ground-based imagers and radar
}

\author{
JENNY ARCHER
}

Stockholm 2009

Space and Plasma Physics

School of Electrical Engineering

Royal Institute of Technology (KTH)

XR-EE-SPP 2009:002 



\begin{abstract}
High resolution data from the ground-based low light auroral imager ASK (Auroral Structure and Dynamics) are used, in combination with EISCAT radar data, to identify and investigate black aurora. Two sets of spectral data originating from emissions caused by high and low energy electrons make it possible to relate the characteristics of different black structures to the energy of the precipitation.

Black auroras are defined as disctrete structures, embedded in diffuse aurora, which exhibit a lack of luminosity. They often have the form of narrow east-west aligned arcs, some of which have curls or shear. Black patches, arc segments and rings, mainly eastward drifting, are observed. Black aurora is also observed together with pulsations. Several examples of these phenomena are analysed.

The ratio between the intensities of the low and high energy emissions measured by ASK is determined. The reductions of high energy particles versus low energy particles in regions of black aurora are investigated.
\end{abstract}


Acknowledgements I would particularly like to thank my supervisors Hanna Dahlgren and Nickolay Ivchenko for their guidance and support, and for always being encouraging and enthusiastic. Thanks to all the people at Space and Plasma Physics for providing such an inspirational, friendly and cheerful atmosphere. A big thank you to Björn Gustavsson and Dan Whiter for help with radar and programming issues. Last but not least, I would like to thank my family for their love and support. 


\section{Contents}

1 Introduction - the Aurora 1

1.1 Auroral Emissions Occur in the Ionosphere . . . . . . . . . . . . . . 1

1.2 Magnetospheric Origins of Precipitating Particles . . . . . . . . . . 1

1.3 The Auroral Ovals . . . . . . . . . . . . . . . . . . . . 3

1.4 Diffuse and Discrete Aurora . . . . . . . . . . . . . . . 3

2 Black Aurora $\quad 4$

2.1 Different Types of Black Aurora . . . . . . . . . . . . . . 5

2.1.1 Black patches, arc segments and rings ......... 5

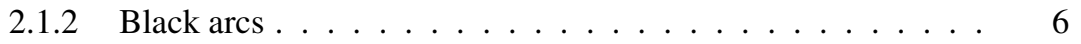

2.1.3 Black vortex streets, curls ............. 6

2.1.4 Pulsations in conjunction with black aurora . . . . . . . . 7

2.2 Theories of Black Aurora . . . . . . . . . . . . . . . . . 7

2.2.1 Coupled ionospheric-magnetospheric generation mechanism . 7

2.2.2 Magnetospheric generation mechanism . . . . . . . . . . 9

2.2.3 Recent findings . . . . . . . . . . . . . . 9

3 Instrumentation $\quad 12$

3.1 The ASK Instrument . . . . . . . . . . . . . . . . . . . . . . 12

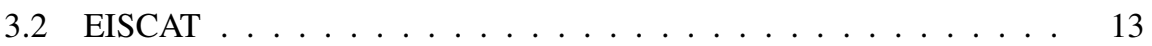

4 Observation 14

4.1 Day 1: 17 October $2006 \ldots \ldots \ldots$

4.2 Day 2: 22 October $2006 \ldots \ldots \ldots$. . . . . . . . . . . . . . . . . . . . . . . . . . 17

4.3 Day 3: 28 October $2006 \ldots$. . . . . . . . . . . . . . . . . . . . . . . . . . . . . . . . . . . .

4.4 Day 4: 31 October $2006 \ldots \ldots \ldots$

4.5 Day 5: 7 February $2007 \ldots \ldots . \ldots 24$

4.6 Day 6: 9 March $2007 \ldots \ldots 26$

5 Analysis 31

5.1 The Auroral Signal . . . . . . . . . . . . . . . . . 31

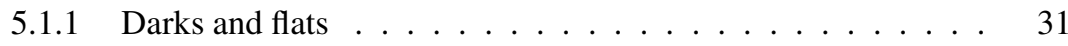

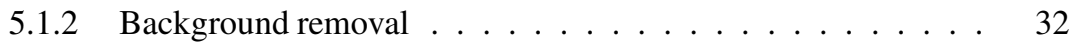

5.1 .3 Noise reduction . . . . . . . . . . . . . 33 
5.2 Determining the Absolute Intensity . . . . . . . . . . . . . . . . . . 34

5.3 The Intensity Ratio . . . . . . . . . . . . . . . . . . . . . . . 34

5.4 Ion chemistry model . . . . . . . . . . . . . . . . 36

5.5 Comparison of optical and radar data . . . . . . . . . . . . . 37

6 Results and Discussion $\quad 38$

6.1 Summary of the Observed Events . . . . . . . . . . . . . . 38

6.2 Absolute Intensity of Black Aurora . . . . . . . . . . . . . . . . . 40

6.2.1 Intensity measurements of pulsating events . . . . . . . . 41

6.3 Intensity Ratios . . . . . . . . . . . . . . . . . . . . . . 43

6.4 Estimated Energy of Precipitating Electrons . . . . . . . . . . . . . . 46

6.5 Comparison of Optical and Radar Data . . . . . . . . . . . . . . . 47

7 Conclusions 


\section{Chapter 1}

\section{Introduction - the Aurora}

The aurora borealis and aurora australis are spectacular natural phenomena which can be observed in the northern and southern winter skies. Many aspects of the aurora are not fully understood. This thesis will focus on black aurora, a form of auroral fine structure.

\subsection{Auroral Emissions Occur in the Ionosphere}

The ionosphere is the uppermost region of the earth's atmosphere. It is located at altitudes of approximately $50-1000 \mathrm{~km}$. The ionosphere consists predominantly of neutral particles, the most common are $\mathrm{N}_{2}, \mathrm{O}_{2}$ and $\mathrm{O}$. However, there is also a lowerdensity plasma component which is mainly generated by photo-ionization from solar radiation, but also by impact ionization caused by precipitating cosmic and magnetospheric particles. The ionosphere is made up of several layers, i.e. the $\mathrm{D}(<90 \mathrm{~km}), \mathrm{E}$ $(90-130 \mathrm{~km})$, and $\mathrm{F}(>130 \mathrm{~km})$ layers, which have distinctly different properties.

Auroras are produced when precipitating magnetospheric particles, mainly electrons, interact with and excite ionospheric atoms and molecules in the $\mathrm{E}$ and $\mathrm{F}$ layers. When the ionospheric particles de-excite light is emitted, and the optical phenomena of the aurora is created.

The auroral spectrum is made up of the atomic lines and molecular bands of the ionospheric particles. However, the energy of the precipitating particles, as well as the density of the ionospheric constituents at the interaction altitude, determine the type of auroral emissions that are produced at a particular time. This is because the densities of the various ionospheric constituents change with altitude and time of day, while highenergy precipitating particles can penetrate to lower altitudes than low-energy particles.

\subsection{Magnetospheric Origins of Precipitating Particles}

Figure 1.1 gives a two-dimensional view of the earth's magnetosphere. If it were not for the solar wind, the earth would have an almost perfect dipole magnetic field. The 


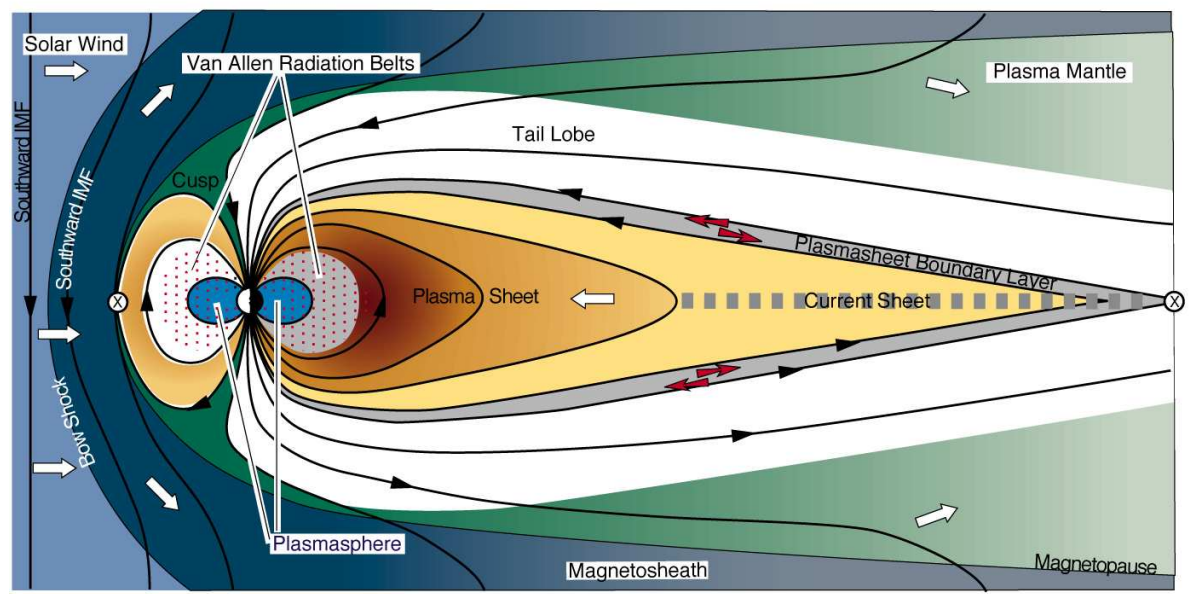

Figure 1.1: Two-dimensional view of the magnetosphere, from Weiss et al. (1992).

solar wind distorts the magnetosphere, compressing it on the dayside and causing it to extend to great distances on the nightside.

Several different plasma populations reside within the magnetophere. The particles which give rise to the nightside aurora are found in the (central) plasma sheet and the plasma sheet boundary layer. While the tail lobes have open field lines, both the central plasma sheet and the plasma sheet boundary layer are characterised by closed magnetic field lines.

The charged plasma particles are guided along these magnetic field lines, and are mirrored back and forth above the geomagnetic poles where the field lines converge. If the parallel velocity of a particle is large enough its pitch angle will be small, and its mirror point may be at such a low altitude that it is lost into the ionosphere where it can excite ionospheric particles.

Different acceleration processes play an important part in increasing the flux and energies of precipitating particles. Two examples are particle-wave interactions which cause particle acceleration, and negative potential structures with converging electric fields that accelerate electrons toward the earth.

These converging electric fields also give rise to shear flows within the plasma. The transversal electric field components point inwards, which means that there exists a discontinuous point or boundary where the direction of the field changes by $180^{\circ}$. A satellite crossing the converging field from north to south would first detect a southward transversal electric field, which abruptly changes to a northward electric field. The $\mathbf{E} \times \mathbf{B}$ plasma drift is therefore oppositely directed on either side of the discontinuity. In the case of a radially converging field, the discontinuity point produces a rotational flow, or vorticity. 


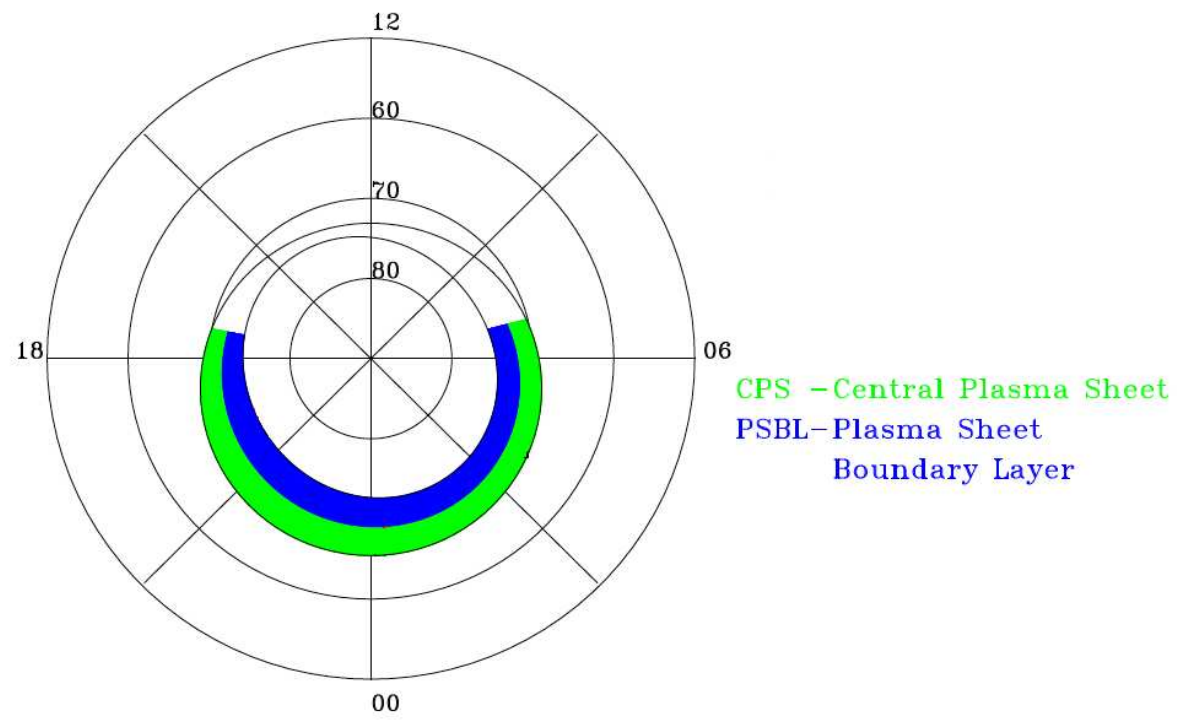

Figure 1.2: The auroral oval, from Marklund et al. (2004).

\subsection{The Auroral Ovals}

Electrons originating in the central plasma sheet and plasma sheet boundary layers precipitate into the nightside auroral oval region. Figure 1.2 shows the parts of the auroral oval onto which these electrons are mapped. The auroral ovals are positioned above the geomagnetic north and south poles. These are the regions in which the aurora occur. The auroral ovals can expand and contract, depending on the level of geomagnetic activity.

Figure 1.2 shows the location of the auroral oval centred roughly above the geomagnetic pole. The given coordinates are geomagnetic latitude and geomagnetic time. The auroral oval always has the same orientation with respect to the sun, while the earth rotates beneath it.

\subsection{Diffuse and Discrete Aurora}

Diffuse aurora exhibits uniform luminosity, and has no (observable) internal structure. It is produced by central plasma sheet particles which undergo pitch angle diffusion without being accelerated. Diffuse aurora is normally observed on the equatorward edge of the nightside auroral oval [Kivelson and Russell, 1995].

Discrete aurora is situated poleward of the diffuse aurora (before midnight), and often has the form of long east-west aligned luminosity bands. Parallel electric fields are believed to accelerate the electrons which cause these discrete auroral arcs. Examples of discrete, small-scale auroral phenomena are curls, rays, and multiple thin arcs. 


\section{Chapter 2}

\section{Black Aurora}

Black aurora was first described in the mid-1970s as "dark holes against a diffuse background" [Parsons and Thomas, 1973, Oguti, 1975]. Numerous observations of a variety of dark regions such as black arcs, vortex streets and patches were subsequently made, most commonly in the magnetic midnight sector. Many features of black aurora have their counterparts within the bright auroral phenomena; therefore, black aurora is often considered the 'negative' of normal aurora [Marklund et al., 1997, Trondsen and Cogger, 1997].

What constitutes black aurora? For example, is the dark region between two discrete bright arcs black aurora? No, not according to the following definition [Royrvik, 1976, Davis, 1978]:

Definition. Black auroras are well-defined, discrete structures exhibiting a lack of luminosity which are embedded in diffuse aurora. In the case of sheared black aurora, the surrounding aurora may be intermediate between diffuse and discrete.

Occasionally, it can be difficult to determine whether an event is made up of black structures upon a bright background or bright structures upon a dark background. The

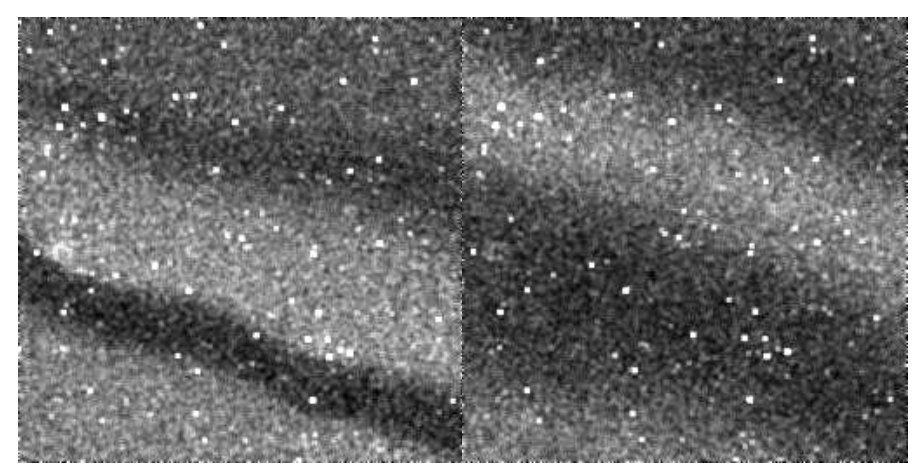

Figure 2.1: Evolution of a dark region, 23 s elapse between the first and second frames. 
frame to the left in Figure 2.1 shows a black arc which seems to have a small degree of shear. $23 \mathrm{~s}$ later, the same black arc has lost definition and widened to become merely a dark region adjacent to a bright arc. Does this event qualify as black aurora? This is almost impossible to determine given the narrow field of view of the auroral imager.

\subsection{Different Types of Black Aurora}

There appear to exist two distinct types of black aurora. The key difference between them is that the first type, comprising black patches, arc segments and rings, shows no sign of any underlying shear mechanism while the second type, comprising black vortex streets and black arcs with curls, exhibits clear evidence of shear.

Unsheared black arcs seem to represent an intermediate state between the two categories. They occasionally develop curls and may transform into full-blown black vortex streets. On the other hand, black patches and arc segments have been observed to metamorphose into black arcs.

Peticolas et al. (2002) summarized the scale sizes of black auroral structures as follows:

- Widths, as well as minor axes of black patches, range from $100 \mathrm{~m}$ to $5 \mathrm{~km}$.

- Major axes of black patches and rings range from 2 to $14 \mathrm{~km}$, while arc segments are generally 20 to $30 \mathrm{~km}$ long.

- The lengths of black arcs and vortex streets cannot be determined since they extend beyond the field of view of most auroral imagers, $>40 \mathrm{~km}$.

All types of black aurora are most frequently observed in the midnight sector. They ususally have an east-west alignment, and drift speeds near $1 \mathrm{~km} / \mathrm{s}$.

\subsubsection{Black patches, arc segments and rings}

Black patches are roughly elliptical regions of black aurora. Black rings have the same basic shape as black patches, but enclose an island of bright aurora. Black arc segments are long, narrow regions of black aurora. These objects display no vorticity; however, small ripples along their edges appear to be commonplace. They are most often observed embedded in the midnight sector diffuse aurora.

These three black auroral forms behave very similarly, and have been observed to evolve from one form into another. For example, black patches have been seen to evolve into black arc segments, and vice versa. They are usually observed in eastward drifting groups together with other black patches, rings and/or arc segments. Although rarely, these black structures have also been observed drifting westward [Kimball and Hallinan, 1998a, Blixt et al., 2005].

Kimball and Hallinan (1998a) determined the average drift speed of 24 patches to be approximately $1.5 \mathrm{~km} / \mathrm{s}$, assuming an altitude of $100 \mathrm{~km}$, while the average speed of 13 black rings was determined to be ca $1.3 \mathrm{~km} / \mathrm{s}$. Trondsen and Cogger (1997) determined the eastward drift speed of 31 patches and arc segments to be mainly concentrated in the range $0.6-1.5 \mathrm{~km} / \mathrm{s}$ at $105 \mathrm{~km}$ altitude. 
Black patches, arc segments and rings appear to be relatively stable. The geometry and drift speed of these structures can remain fairly constant for up to a minute. However, observations have also yielded examples of arc segments and patches which rapidly change shape and/or direction.

\subsubsection{Black arcs}

Black arcs are long, narrow bands of black aurora, usually extending in the east-west direction. Single or multiple, quasi-parallel black arcs are most frequently observed in the midnight sector diffuse aurora. It is not always possible to determine whether smooth black arcs have an eastward or westward drift, however, they often travel equatorward at a speed of a few hundred metres per second.

As in the case of black patches, arc segments and rings, small-scale ripples are frequently observed moving along the edges of black arcs. In addition, irregular and unstable black arcs which exhibit, for example, folds and kinks have also been observed.

There seems to be a connection between black arcs and black patches, arc segments and rings. Royrvik (1976) observed easward drifting black patches joining together to form east-west aligned black arcs. Other observations showed structures which were intermediate between a black arc and an eastward drifting string of black patches [Trondsen and Cogger, 1997].

Conversely, black arcs have been observed to develop curls (vortices) and also to transform into black vortex streets.

\subsubsection{Black vortex streets, curls}

Black vortex streets are defined as a number of curls or vortices spaced quasi-periodically along a black arc [Trondsen and Cogger, 1997]. Without exception, all black curls and vortices observed so far have a clockwise rotational sense when viewed anti-parallel to the magnetic field. This is opposite to the rotational sense of bright auroral curls which always exhibit counter-clockwise vorticity.

Black vortex streets and black arcs exhibiting curls usually extend in the east-west direction. They most frequently occur within midnight sector diffuse, or semi-diffuse, aurora. As described above, curls and vortex streets have been observed to develop on smooth black arcs.

Kimball and Hallinan (1998b) analysed 27 black vortex street events and determined the average wavelength of the black curls to be $2.3 \mathrm{~km}$. Curl systems were observed to drift either eastward or westward along the black arcs with an average speed of $0.9 \mathrm{~km} / \mathrm{s}$.

Black vortex streets seem to occur rarely when compared to the observed frequency of other black auroral structures. They also have much shorter lifetimes than e.g. black patches, often existing for only a few seconds or less. 


\subsubsection{Pulsations in conjunction with black aurora}

Black aurora and pulsating aurora are sometimes observed simultaneously. The only exception is sheared black aurora which has never been observed in conjunction with pulsations.

Kimball and Hallinan (1998a) described the following pattern of events as viewed on several occasions through an all-sky camera: Westward drifting pulsating aurora gradually moved equatorward. Immediately northward of the pulsating aurora was non-pulsating eastward drifting diffuse aurora which, upon nearing the magnetic zenith, was observed to contain eastward drifting black structures. Occasionally, the pulsations and the black structures overlapped. Sometimes, black vortex streets were seen to the north of the eastward drifting black structures; however, the pulsations never overlapped with these sheared black auroras.

Kimball and Hallinan (1998a) observed a phenomenon, termed veiling, during which the pulsations added more to the intensity of the black structures than to the intensity of the surrounding diffuse aurora. In the event of total veiling, the black structures attained the same intensity as the diffuse aurora and became invisible. They hypothesized that total veiling suggested an intensity limit, possibly associated with the strong pitch angle diffusion limit.

Another hypothesis is that of dual-layer pulsations [Trondsen and Cogger, 1997] which involves two overlapping layers of aurora. The background layer comprises diffuse aurora containing eastward drifting black structures. The foreground layer is made up of westward drifting pulsating aurora, possibly also containing black aurora. The two auroral layers may be generated by different precipitation mechanisms giving rise to two sets of particles with different energy distributions and emission altitudes.

\subsection{Theories of Black Aurora}

The underlying mechanisms which generate black aurora are not fully understood, although two major theories explaining the phenomenon exist.

\subsubsection{Coupled ionospheric-magnetospheric generation mechanism}

Marklund et al. (1994) reported the detection of extremely intense (1 V/m) and smallscale $(1 \mathrm{~km})$ diverging electric fields by the Freja satellite. They discovered that these electric field structures, associated with an excess of positive space charge, are frequent occurrences at altitudes between 800 and $1700 \mathrm{~km}$, i.e. below the auroral acceleration region.

In conjunction with the narrow, diverging electric fields, Marklund et al. (1994, 1997) detected dropouts of precipitating electrons, downward field-aligned currents of upward flowing thermal electrons, precipitating or transversely energised ions, and ionospheric density depletions. They proposed that the diverging electric field structures were an indication of the presence of black arcs and black vortex streets.

They found two categories of diverging electric fields; radially diverging fields with scale size and spacing consistent with that of black vortex streets, and north-south 


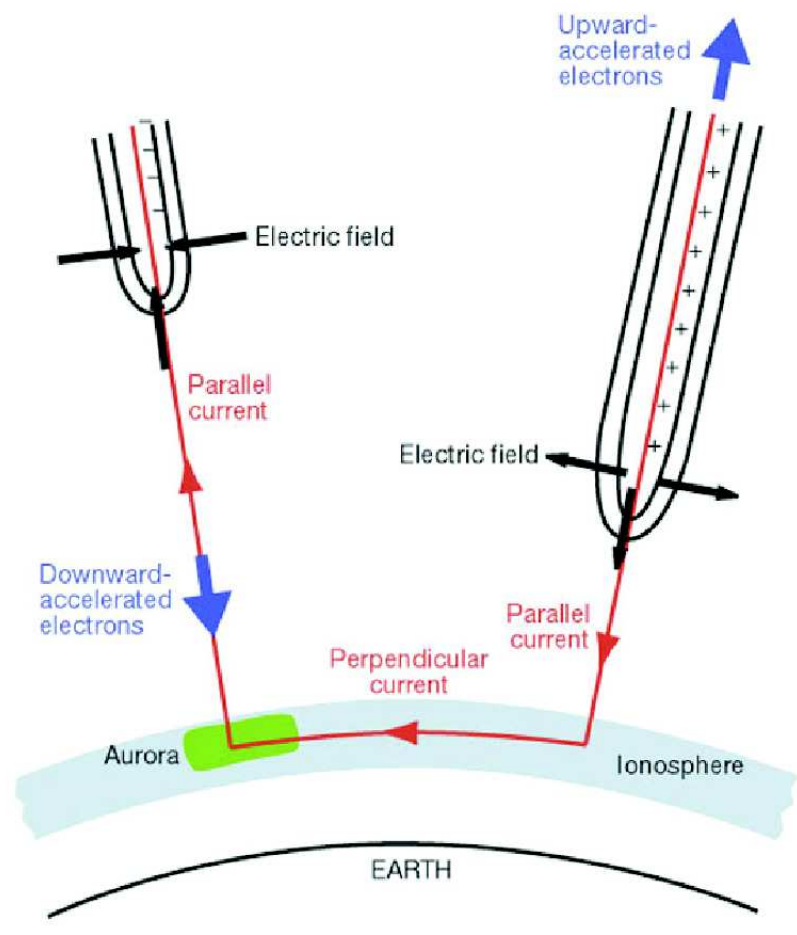

Figure 2.2: Schematic drawing of inter-connected positive and negative potential structures, from Marklund et al. (2001).

oriented diverging fields which were believed to be connected with black arcs.

Marklund et al. (1997) suggested that the diverging electric fields observed by Freja are associated with narrow (1-2 km), low-altitude, positive potential structures which are connected to the higher altitude, negative potential structures, known to accelerate precipitating auroral electrons, see Figure 2.2. The positive potential structures constitute return current regions, due to the downward field-aligned currents mentioned above.

Several theoretical models have been put forward to explain the creation of black vortices [Shukla et al., 1995, Johnson and Chang, 1995]. For example, Keskinen and Ganguli (1996) studied the non-linear evolution of the Kelvin-Helmholtz shear instability using parameters typical of black auroral curls. They found that, in the presence of a density gradient, the Kelvin-Helmholtz instability can give rise to structured vortices and electric fields, as well as large density depletions. Their simulation results were thus in good agreement with the experimental observations.

The current, generally accepted theory is that black vortex streets and curls are produced by the Kelvin-Helmholtz instability occurring in regions of positive space 
charge (diverging electric field) [Blixt et al., 2005]. This explains why the rotational sense of black curls is opposite to that of bright curls which are widely thought to be caused by the Kelvin-Helmholtz instability occurring in regions of negative space charge (converging electric field).

\subsubsection{Magnetospheric generation mechanism}

Peticolas et al. (2002) used aircraft-based optical observations, as well as plasma and field measurements originating from the FAST satellite, at ca $4000 \mathrm{~km}$ altitude, to study black aurora. They compared data from one night on which black aurora was observed with data from six nights when no black aurora was detected.

FAST did not cross the magnetic zenith simultaneously with the optical observations of black aurora; therefore, based on previous research, significant $(>50 \%)$, narrow decreases in the uniform, downward electron energy flux were interpreted to constitute satellite crossings over black aurora. FAST detected two such electron energy flux decreases.

Peticolas et al. (2002) compared the electron differential energy flux at pitch angles of $0^{\circ}\left(0 \pm 22^{\circ}\right)$ and $90^{\circ}\left(90 \pm 22^{\circ}\right)$. The two narrow regions assumed to be black aurora exhibited differential energy fluxes which were depleted in the downward loss cone $\left(0 \pm 22^{\circ}\right)$ at energies $>2 \mathrm{keV}$, creating a partial double loss cone distribution, see Figure 2.3. In the surrounding diffuse aurora, the electron differential energy fluxes were isotropic at all energies, creating a single loss cone distribution.

The differential energy fluxes of the diffuse and black aurora were identical in the mirroring electron population $\left(90 \pm 22^{\circ}\right)$, and differed in the precipitating electron population $\left(0 \pm 22^{\circ}\right)$ only at energies $>2 \mathrm{keV}$. Therefore, Peticolas et al. (2002) reasoned that electrons were not accelerated differently above regions of black aurora than above diffuse aurora. The mechanism which generates black aurora acts only on the precipitating fluxes at energies $>2 \mathrm{keV}$, causing a suppression of the strong pitch angle diffusion at high energies.

On the six nights without black aurora, the partial double loss cone distribution was found to be a common occurrence in the central plasma sheet region of the auroral oval. Conversely, the single loss cone distribution, which exhibits strong pitch angle diffusion at all energies, was dominant only on the night with black aurora. The temperature of the central plasma sheet electrons was significantly higher on the night with black aurora than on the nights without black aurora.

Signatures of the intense diverging electric fields, upward moving electrons, and downward currents described in the previous section were found on the equatorward boundary of the auroral oval on all seven nights, and on the poleward boundary on three nights. However, there was no evidence that these events were connected to black aurora.

\subsubsection{Recent findings}

Blixt and Kosch (2004) performed coordinated optical and incoherent scatter radar observations of two unsheared black arcs. They found that the ionospheric electron density did not decrease when the black arcs crossed the radar beam. A downward field- 

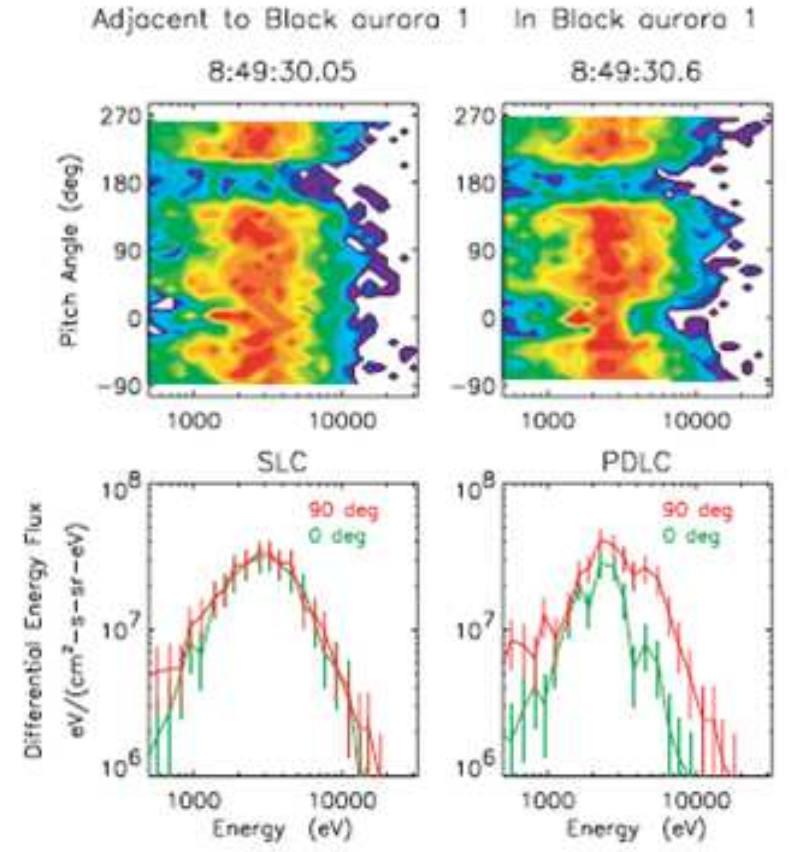

Figure 2.3: Electron differential energy fluxes for diffuse and black aurora, showing single loss cone (SLC) and partial double loss cone (PDLC), from Peticolas et al. (2002). 
aligned current would give rise to an ionospheric electron density depletion. Therefore, they concluded that the two unsheared black arcs were not associated with any significant downward field-aligned current.

Blixt et al. (2005) studied the drift speeds of black patches using EISCAT radar data and an auroral TV camera. Their goal was to determine whether the drift speeds of black structures depends on the energy of the precipitating electrons in the surrounding diffuse aurora.

Black aurora may be produced by a magnetospheric blocking mechanism which prevents high energy electrons from entering the loss cone, see Section 2.2.2. If this blocking mechanism gradient-B drifts with the source electron population in the magnetosphere, then the drift speed of black patches should increase with the energy of the precipitating electrons in the surrounding diffuse aurora, since the magnetospheric gradient-B curvature drift is energy dependent.

Blixt et al. (2005) discovered that the drift speeds of eight observed black patches were several $\mathrm{km} / \mathrm{s}$ faster than the ionospheric plasma flow, and that the drift velocity of the observed black patches increased with the energy of the precipitating electrons.

Gustavsson et al. (2008) performed coordinated incoherent scatter radar, bistatic white-light and monostatic spectral image observations of unsheared black aurora. Their results showed that black auroral structures are not completely devoid of emissions; the relative intensity reductions in the observed black structures were $25 \%$ at $4278 \AA$ and $18 \%$ at $8446 \AA$ compared to the surrounding diffuse aurora.

The EISCAT electron density measurements were used to model the optical emissions at $4278 \AA$ and $8446 \AA$. The model showed that the positive potential structure described in Section 2.2.1 would cause a larger relative reduction in $8446 \AA$ emissions than in $4278 \AA$ emissions. This was not in agreement with the observational measurements which showed a larger reduction in $4278 \AA$ emissions.

The magnetospheric blocking mechansim described in Section 2.2.2 provided a good fit to the observational data. Therefore, Gustavsson et al. (2008) concluded that unsheared black aurora is caused by magnetospheric regions of suppressed high-energy electron pitch angle scattering.

Simultaneous optical and electron observations of thirteen black auroral events by the Reimei satellite [Sakanoi et al., 2008] showed depletions in the high-energy ( $>2$ $7 \mathrm{keV}$ ) precipitating electron flux in regions of black aurora. The electron flux at lower energies was found to be almost identical for the black aurora and the surrounding diffuse aurora. This agrees with the results of Peticolas et al. (2002) described above. 


\section{Chapter 3}

\section{Instrumentation}

\subsection{The ASK Instrument}

The ASK (Auroral Structure and Kinetics) instrument comprises three narrow field low light imagers and two photometers; the cameras are Andor iXon DV887 backilluminated EMCCDs producing images with a resolution of $512 \times 512$ pixels.

The function of ASK is to record multi-spectral images of small-scale auroral phenomena using different narrow passband interference filters. ASK's high spatial and temporal resolution provide the means to study the dynamics and morphology of auroral fine structure, while imaging aurora through different filters makes it possible to relate auroral characteristics to the energy of the precipitation.

For the purpose of studying black aurora, images acquired with two different filters were analysed. The first filter has a central wavelength of $6730 \AA$ and a FWHM of $140 \AA$, and is used to detect $\mathrm{N}_{2}$ emissions originating from high-energy electron precipitation. The second filter has a central wavelength of $7774 \AA$ and a FWHM of $15 \AA$. It detects OI emissions which mostly originate from low-energy electron precipitation [Dahlgren, 2008]. The filter transmissions curves are shown in Figure 3.1.

Binning can be used to reduce the amount of data, therefore making it possible to
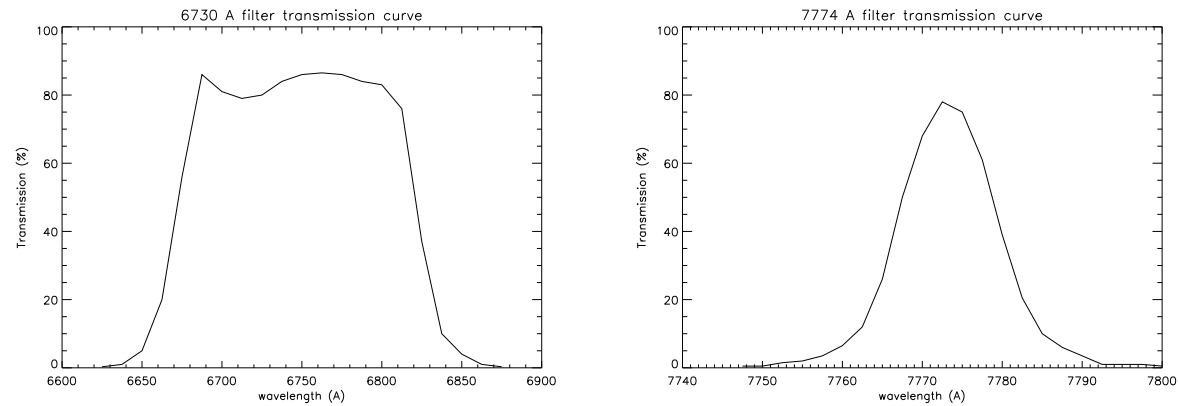

Figure 3.1: Filter transmission curves, $6730 \AA$ (left) and $7774 \AA$ (right). 
increase the number of frames per second. For the events described in this study, the frame rate is $10-32 \mathrm{~Hz}$, the image size is $256 \times 256$ pixels, and the field of view is $3.1^{\circ} \times 3.1^{\circ}$.

\subsection{EISCAT}

The European Incoherent Scatter radar, EISCAT, is stationed outside Troms $\varnothing$, Norway. EISCAT performs measurements of the ionospheric electron density, electron and ion temperatures, and the ion drift velocity. The radar beam has a full opening angle of about $0.6^{\circ}$, which corresponds to approximately $1 \mathrm{~km}$ at an altitude of $105 \mathrm{~km}$ [Blixt and Kosch, 2004].

EISCAT measurements of the ionospheric electron density were available for five events (two nights). The ionospheric electron density was compared to the optical data provided by ASK. EISCAT was pointing along the local magnetic field line during the studied events. 


\section{Chapter 4}

\section{Observation}

The data presented in this thesis were recorded during the observational campaign of 2006/2007 when the ASK instrument was stationed at the EISCAT radar site in Ramfjordmoen, Norway $\left(69.59^{\circ} \mathrm{N}, 19.23^{\circ} \mathrm{E}\right)$. The cameras pointed towards the magnetic zenith (azimuth $185.8^{\circ} \mathrm{E}$ of $\mathrm{N}$, elevation $76.9^{\circ}$ ). The field of view was $3.1^{\circ} \times 3.1^{\circ}$ which corresponds to approximately $5 \mathrm{~km} \times 5 \mathrm{~km}$ at an altitude of $100 \mathrm{~km}$. Two passband filters, $6730 \AA$ and $7774 \AA$, were fitted to ASK1 and ASK3 respectively to obtain simultaneous images of aurora in two different spectral bands; ASK1 was used to measure high-energy electron precipitation, and ASK3 low-energy electron precipitation.

The keogram catalogue at ask.esr.eiscat.no presents the ASK data in condensed form. Each 20-minute image sequence recorded by ASK is abridged to a keogram by placing the central column of pixels from each mean image (average image over 2 seconds) next to each other from left to right. Keograms from both the Troms $\emptyset$ and Svalbard campaigns were searched for potential black aurora candidates. The brightest images, acquired from ASK1, were then viewed to determine which of the potential events actually constituted black aurora. Sixteen events recorded during the abovementioned 2006/2007 campaign were finally selected for further analysis, see Table 4.1. No black aurora events were found in the Svalbard data.

ASK records aurora occurring in a tiny part of the sky in excellent spatial and temporal detail. According to the definition, shearless black aurora is found within regions of diffuse aurora. However, aurora which appears diffuse in the small field of view of the ASK camera may very well be part of a discrete structure when observed by an all-sky camera or satellite, and vice versa, the fine-structured, discrete aurora observed by ASK may be perceived as featureless and diffuse when observed in a whole sky context [Trondsen, 1998].

The intensity varied substantially from event to event. For example, the intensity of a black structure in Event 6E was approximately $900 \mathrm{R}$ in ASK1, i.e. $50 \%$ higher than the intensity of the surrounding bright aurora in Event $2 \mathrm{C}$ which was approximately $600 \mathrm{R}$ in ASK1. However, the appearance and behaviour of the dark structures in both events were similar, see Figure 4.1.

Taking these considerations into account, the criteria chosen for an event to qualify as black aurora were the following: 


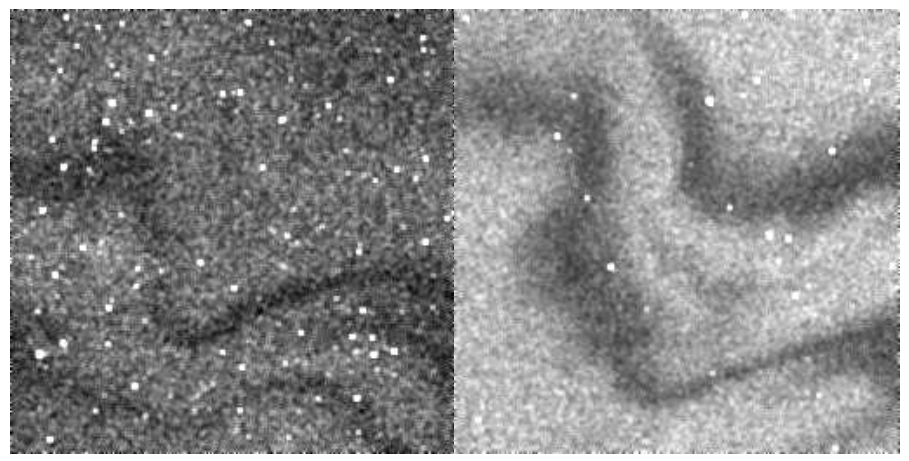

Figure 4.1: Frame from Event 2C (left) and Event 6E (right).

1. The surrounding aurora should appear diffuse, or intermediate between diffuse and discrete in the case of sheared events, in the ASK field of view.

2. If a dark stucture had the appearance and dynamics of black aurora as described in the literature [Trondsen and Cogger, 1997, Kimball and Hallinan, 1998a, 1998b] then it should be defined as such, regardless of its intensity.

The number of events discovered which fulfil these criteria is beyond the scope of this thesis. Sixteen representative and/or particularly interesting events were therefore chosen for analysis, see Table 4.1. Two of the selected events, 2A and 6A, did not fulfil the criteria for black aurora. They were included because they comprised discrete dark structures situated adjacent to bright aurora, and could be used for comparison with the proper black auroral events. Event 2D constitutes a limiting case for which it was difficult to determine whether the observed dark structure was a black arc embedded in diffuse aurora or the dark space between two bright arcs.

The average intensity in the whole field of view of each event is given in Rayleighs, Table 4.1. Common for all events is the similar morphology of the black aurora as seen by ASK1 and ASK3. However, the intensity is several times lower in ASK3, due to the ASK3 $7774 \AA$ filter being much narrower $(15 \AA)$ than the ASK1 $6730 \AA$ Ailter (140 ̊). The table also indicates whether EISCAT radar data were available for the selected events.

A brief description together with a sequence of images (recorded by ASK1) of each event follows. The time of the first frame is given as well as the time between frames, $\Delta$ t. All times are given in UT. Local magnetic midnight occurs at approximately 21:30 UT. Compass directions for all images in this chapter are shown in Figure 4.2.

\subsection{Day 1: 17 October 2006}

Diffuse aurora is visible in the ASK field of view from 22:15 to 23:30. Faint, dark eastward drifting patches and arc segments begin to appear at ca 22:35. After a few 


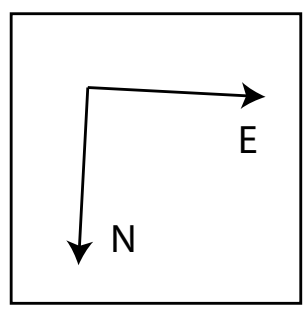

Figure 4.2: Compass directions.

\begin{tabular}{|l|l|l|r|r|l|}
\hline & & & \multicolumn{2}{|c|}{ Mean Intensity, R } & \\
Event & Date & Time, UT & ASK1 & ASK3 & Radar Data \\
\hline \hline & & & & & \\
1A & 20061017 & $22: 37: 17-22: 44: 08$ & 528 & 144 & yes \\
& & & & & \\
2A & 20061022 & $18: 39: 43-18: 39: 53$ & 1163 & 389 & yes \\
2B & & $20: 50: 22-20: 51: 47$ & 541 & 160 & yes \\
2C & & $20: 51: 57-20: 53: 07$ & 488 & 149 & yes \\
2D & & $20: 54: 35-20: 55: 52$ & 558 & 158 & yes \\
& & & & & \\
3A & 20061028 & $20: 24: 19-20: 25: 24$ & 2120 & 524 & no \\
3B & & $20: 31: 37-20: 31: 58$ & 1478 & 371 & no \\
& & & & & \\
4A & 20061031 & $21: 45: 52-21: 46: 16$ & 1361 & 325 & no \\
4B & & $21: 47: 36-21: 47: 40$ & 1071 & 273 & no \\
4C & & $21: 47: 44-21: 47: 58$ & 1130 & 292 & no \\
& & & & & \\
5A & 20070207 & $21: 10: 24-21: 10: 51$ & 1402 & 448 & no \\
& & & & & \\
6A & 20070309 & $22: 10: 28-22: 11: 42$ & 788 & 246 & no \\
6B & & $22: 42: 48-22: 43: 09$ & 603 & 177 & no \\
6C & & $22: 44: 00-22: 44: 30$ & 699 & 211 & no \\
6D & & $23: 05: 32-23: 06: 13$ & 1355 & 485 & no \\
6E & & $23: 07: 37-23: 08: 08$ & 1291 & 466 & no \\
& & & & & \\
\hline
\end{tabular}

Table 4.1: List of Events. 


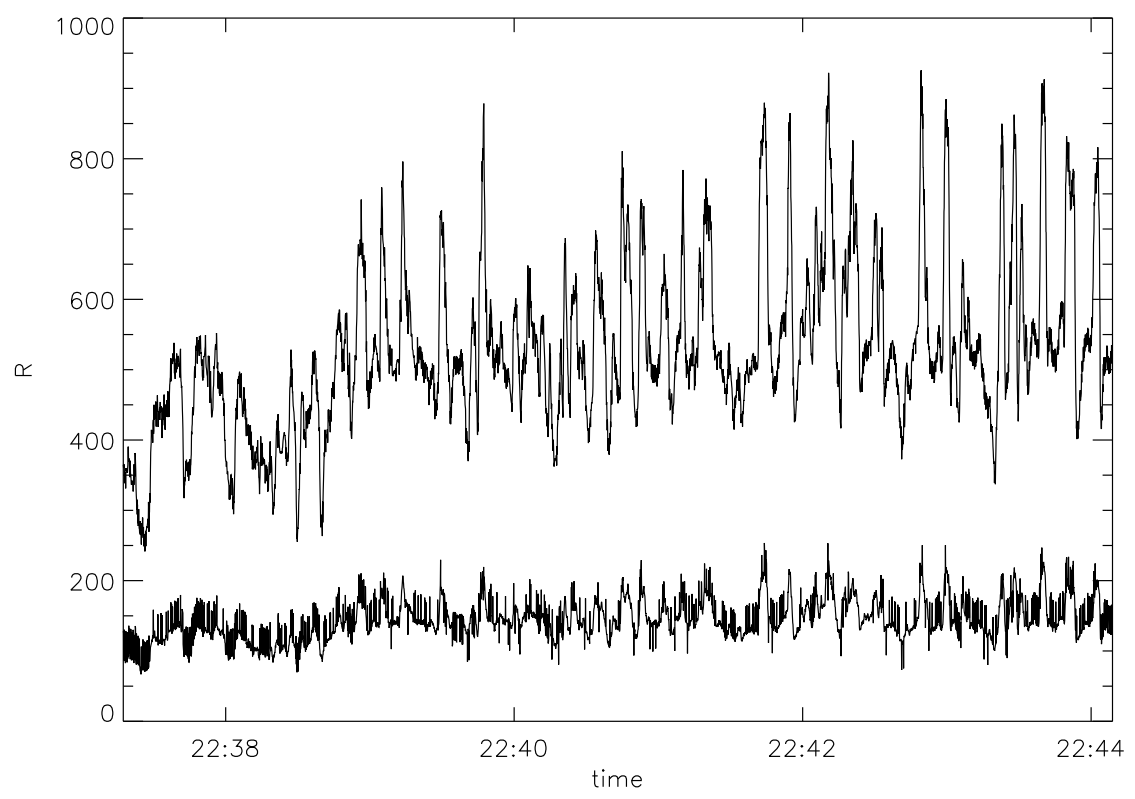

Figure 4.3: Event 1A. Mean intensity in ASK1, ASK3.

minutes, the dark structures become more pronounced.

Event 1A: 22:37 - 22:44. Diffuse aurora appears to be switched off and on, Figure 4.3. When in off-mode, a structured background of quasi-parallel, east-west aligned black arc segments and patches, embedded in a layer of diffuse aurora, is revealed, see Figure 4.4. The arc segments are drifting eastward at approximately $0.9 \mathrm{~km} / \mathrm{s}$. When in on-mode, the black structures are invisible. The pulsations have an approximate frequency of $0.1 \mathrm{~Hz}$.

After 22:44, the black structures grow fainter, less well-defined and finally disappear, although the pulsations continue for some time.

\subsection{Day 2: 22 October 2006}

Event 2A: 18:39. A north-south aligned dark region situated between two bright arcs drifts towards the west, see Figure 4.5. Ripples of approximate wavelength $1.2 \mathrm{~km}$ move southward along its western boundary and northward along its eastern boundary at $2.8 \mathrm{~km} / \mathrm{s}$. According to the definition, this event does not constitute black aurora since the surrounding bright aurora is dynamic and discrete.

Approximately 2 hours later, the dynamic aurora observed in connection with Event 


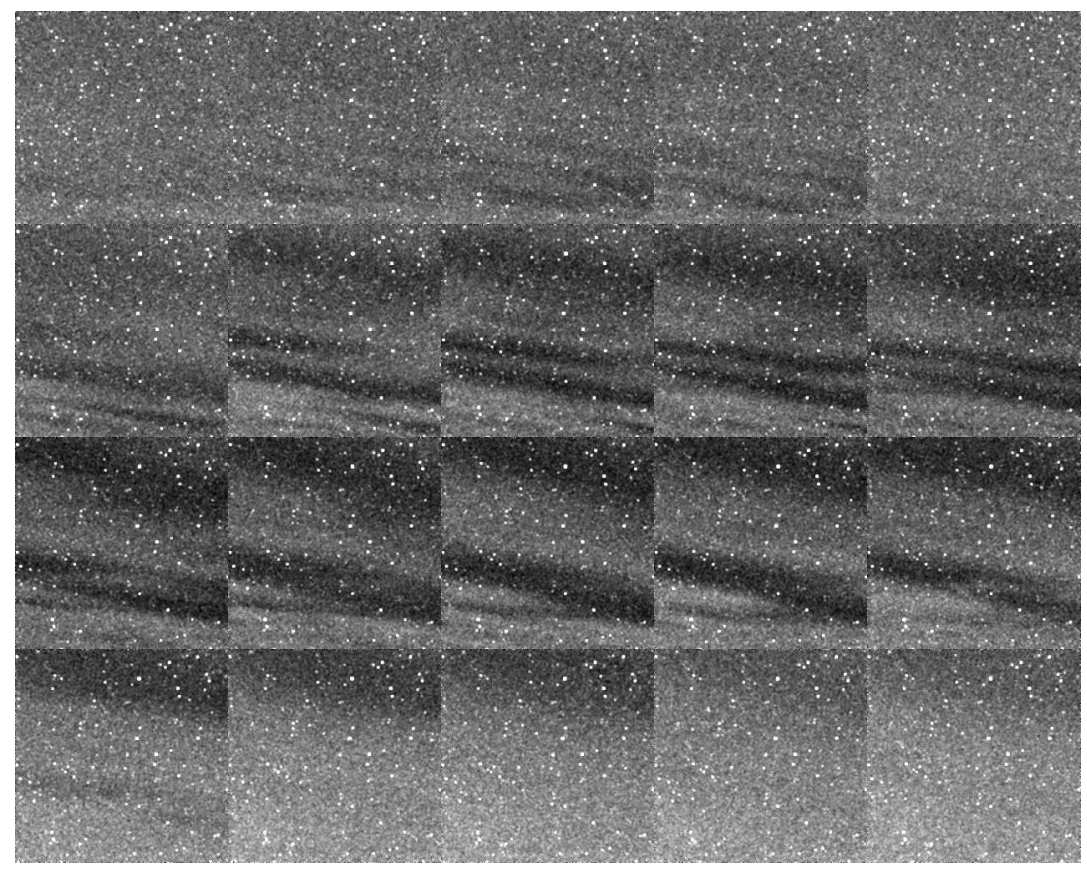

Figure 4.4: Event 1A. 22:37:19.900, $\Delta \mathrm{t}=0.600 \mathrm{~s}$.

$2 \mathrm{~A}$ has been replaced by diffuse aurora. Figure 4.6 is a keogram of Events $2 \mathrm{~B}$ and $2 \mathrm{C}$, in which multiple black arcs are seen to drift slowly equatorward.

Event 2B: 20:50 - 20:51. A dark region at the northernmost edge of the field of view transforms into an east-west aligned black arc, minimum width ca $200 \mathrm{~m}$, which drifts slowly southward across the field of view, see Figure 4.7. The intensity of the surrounding diffuse aurora is higher immediately to the south of the black arc. The black arc exhibits loose curls which seem to be moving toward the west at ca $0.8 \mathrm{~km} / \mathrm{s}$. The wavelength is approximately $2.5 \mathrm{~km}$. $45 \mathrm{~s}$ after first appearing, the arc merges with a second eastward moving quasi-parallel arc. The black arc becomes wider and less well-defined, and eventually fades into the surrounding diffuse aurora.

Event 2C: 20:51 - 20:53. Multiple quasi-parallel, east-west aligned wavy black arcs, some of which are no more than $200 \mathrm{~m}$ wide drift slowly southward, Figure 4.8. The waves are propagating along the arcs toward the west at approximately $0.6 \mathrm{~km} / \mathrm{s}$, and actually resemble waves travelling along a string.

Event 2D:20:54 - 20:55. A $400 \mathrm{~m}$ wide east-west aligned black arc, adjacent to a wider, homogeneous, bright arc to the south, drifts slowly southward across the field of view, see Figure 4.9. The intensity of the bright arc is higher than that of the diffuse aurora seen in Event 2C. At around 20:55:00, the black arc seems to exhibit a small 


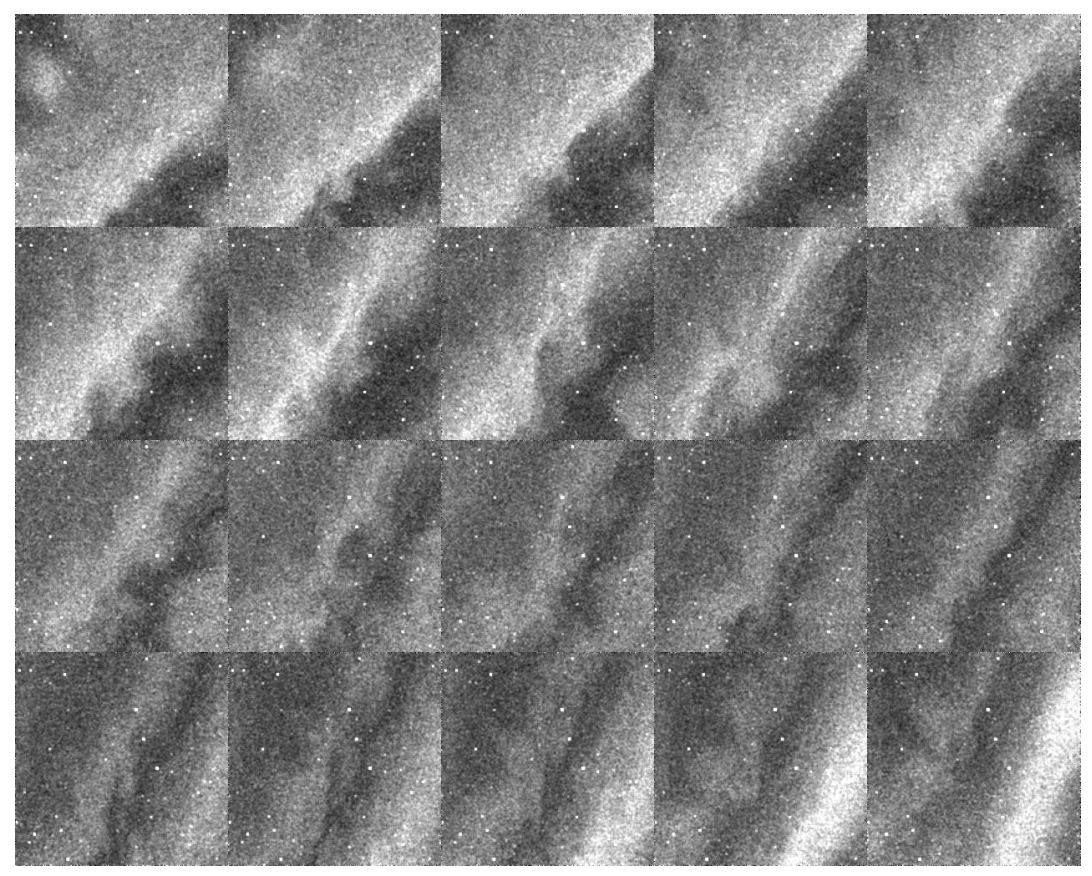

Figure 4.5: Event 2A. 18:39:46.094, $\Delta \mathrm{t}=0.312 \mathrm{~s}$.

degree of shear, before gradually growing wider and losing definition until it resembles a dark region situated between two bright arcs. This event constitutes a limiting case for which it is not possible to determine whether the dark region is a proper black arc or merely the dark space between two bright arcs.

After 22:00, the diffuse aurora is replaced by bright, dynamic aurora which is visible in the field of view for a few minutes, before giving way to pulsating aurora.

\subsection{Day 3: 28 October 2006}

Structured, dynamic as well as diffuse aurora is visible in the ASK field of view from ca 19:40 until 23:00 when it starts to become cloudy.

Event 3A: 20:24 - 20:25. A layer of diffuse aurora switches off several times to reveal a structured background of multiple northeast-southwest aligned black arc segments and patches embedded in diffuse aurora, see Figure 4.11. The aurora is pulsating with a frequency of $0.15 \mathrm{~Hz}$, Figure 4.10. The black structures are dynamic; each time they are revealed, their appearance is different. They seem to drift towards the east, although it is not possible to determine their speed since they are only visible for very short intervals. After approximately $40 \mathrm{~s}$, the diffuse aurora loses intensity and appears to flicker, and the visibility of the dark structures increases somewhat. 


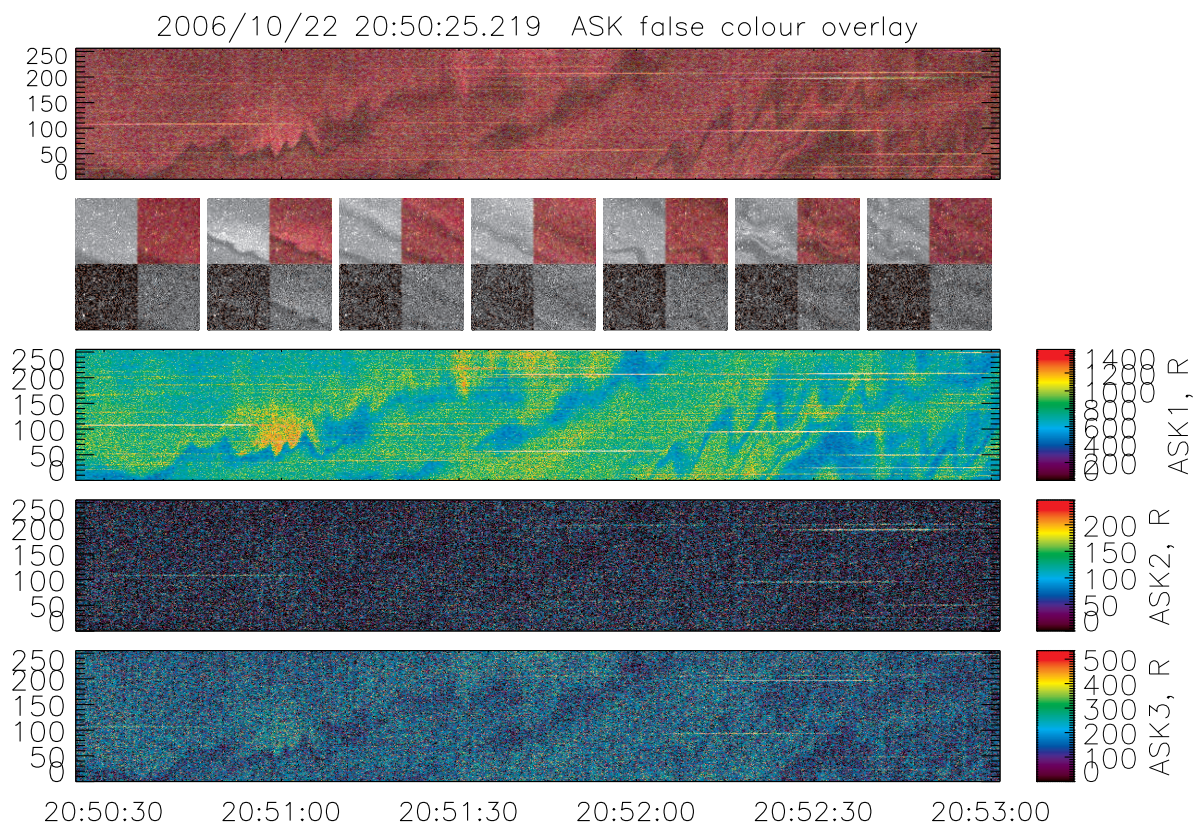

Figure 4.6: Keogram of Events 2B and 2C.

After a few minutes the flickering ceases, and dark patches and arc segments embedded in diffuse aurora drift across the field of view.

Event 3B: 20:31. A $500 \mathrm{~m}$ wide, east-west aligned, eastward drifting dark arc, or arc segment, enters the field of view from the west, while at the same time slowly moving equatorward, Figure 4.12 . The arc gradually broadens to a width of approximately $1.5 \mathrm{~km}$ and grows blacker, and a thin wisp of bright aurora situated within the black arc (a weasel) moves to the east with a velocity of $2 \mathrm{~km} / \mathrm{s}$. Shortly afterwards, at 20:31:58, the tail end of the black arc exits the ASK field of view. The black arc seems to be moving at the same speed as the weasel, which makes it roughly $35 \mathrm{~km}$ long.

\subsection{Day 4: 31 October 2006}

An auroral breakup occurs at around 21:35.

Event 4A: 21:45 - 21:46. Several quasi-parallel, east-west aligned black arc segments exhibiting curls move rapidly westward at $7-8.5 \mathrm{~km} / \mathrm{s}$, Figure 4.13 . One of the arc segments grows wider and evolves into an irregular black patch with fast-moving ripples along its boundaries. The black patch subsequently transforms into a northwest- 


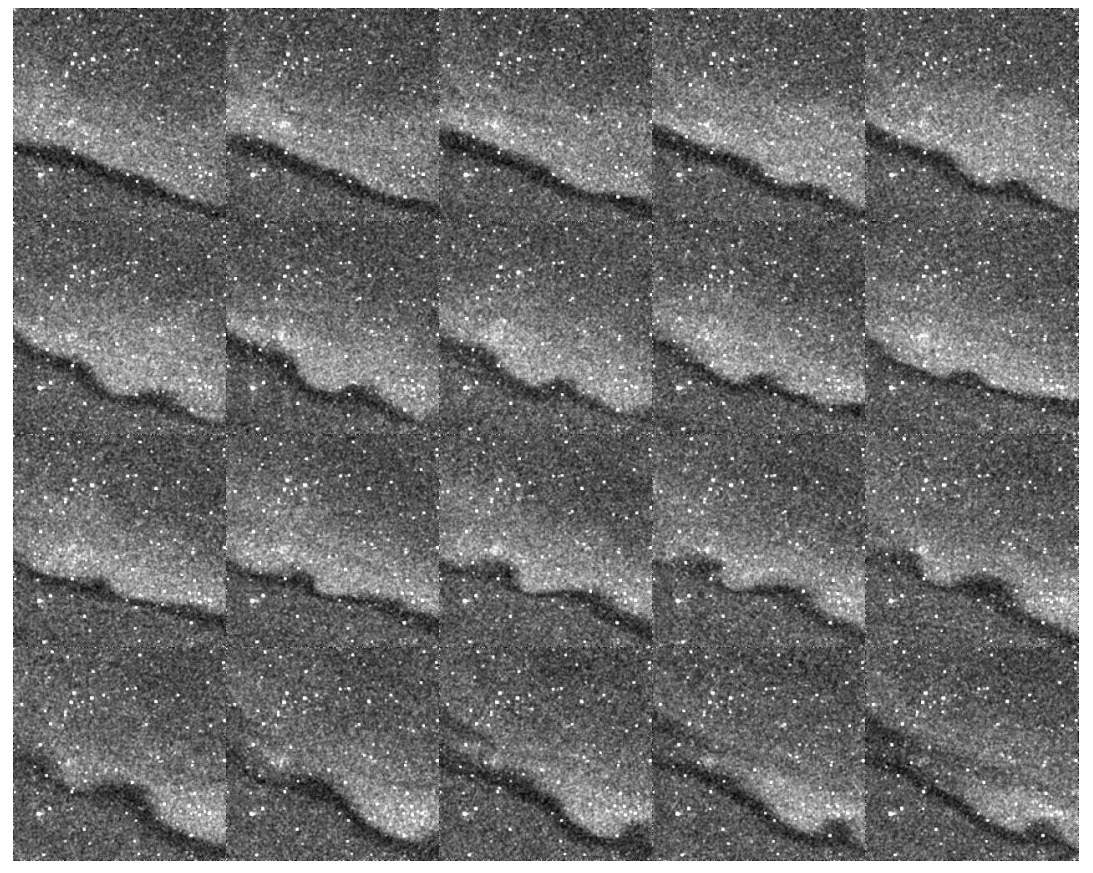

Figure 4.7: Event 2B. 20:50:56.781, $\Delta \mathrm{t}=0.469 \mathrm{~s}$.

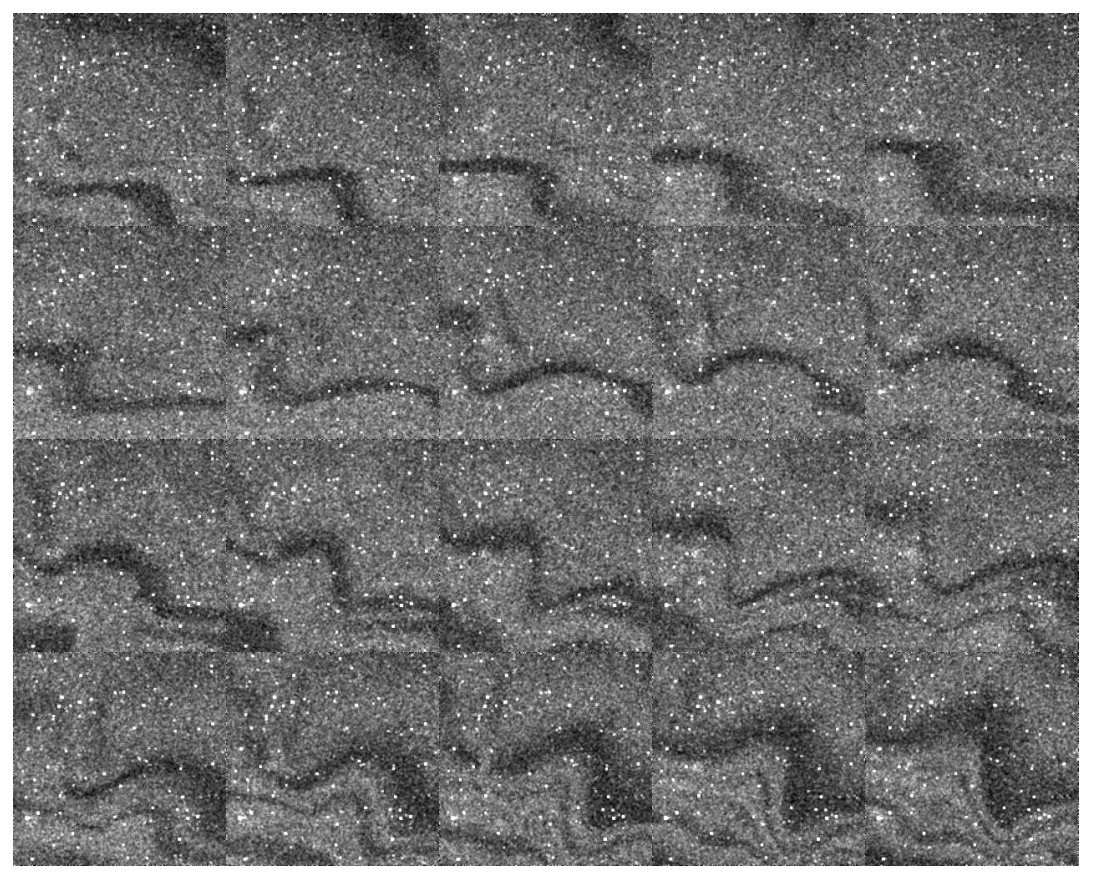

Figure 4.8: Event 2C. 20:52:05.219, $\Delta \mathrm{t}=0.906 \mathrm{~s}$. 


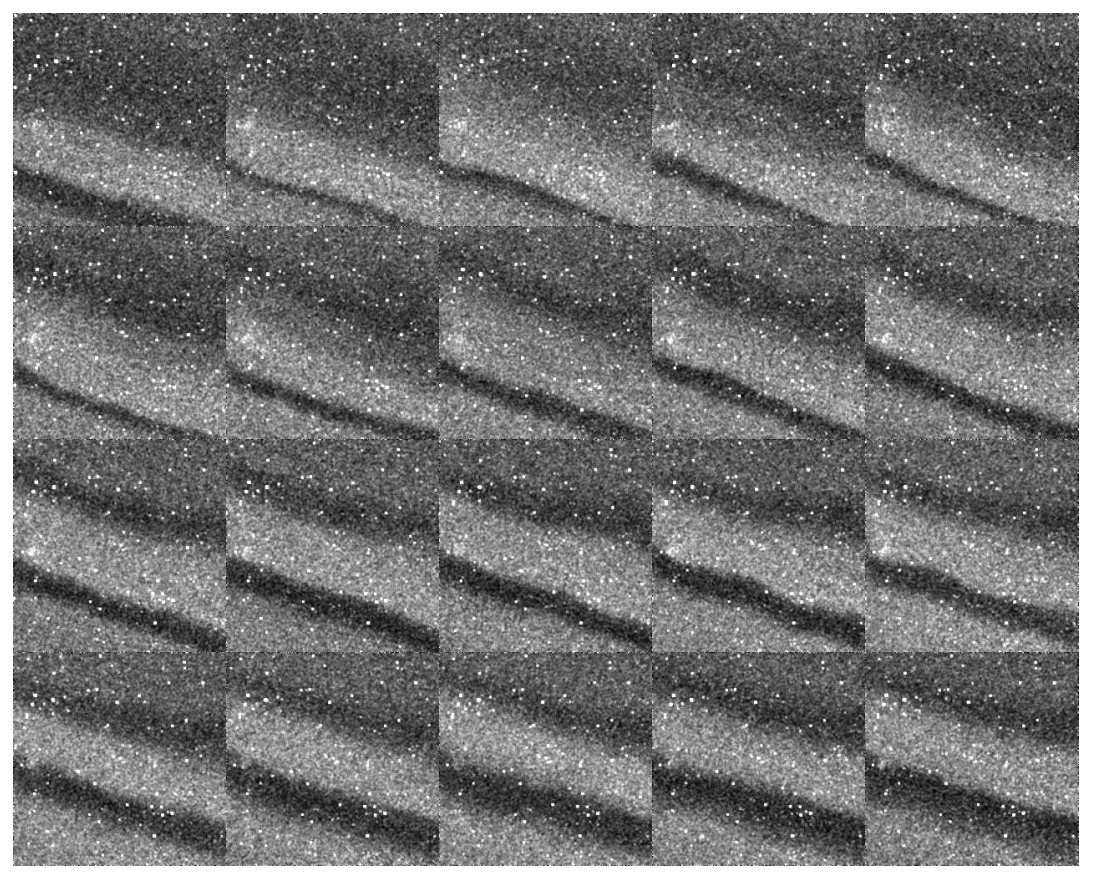

Figure 4.9: Event 2D. 20:54:46.469, $\Delta \mathrm{t}=1.25 \mathrm{~s}$.

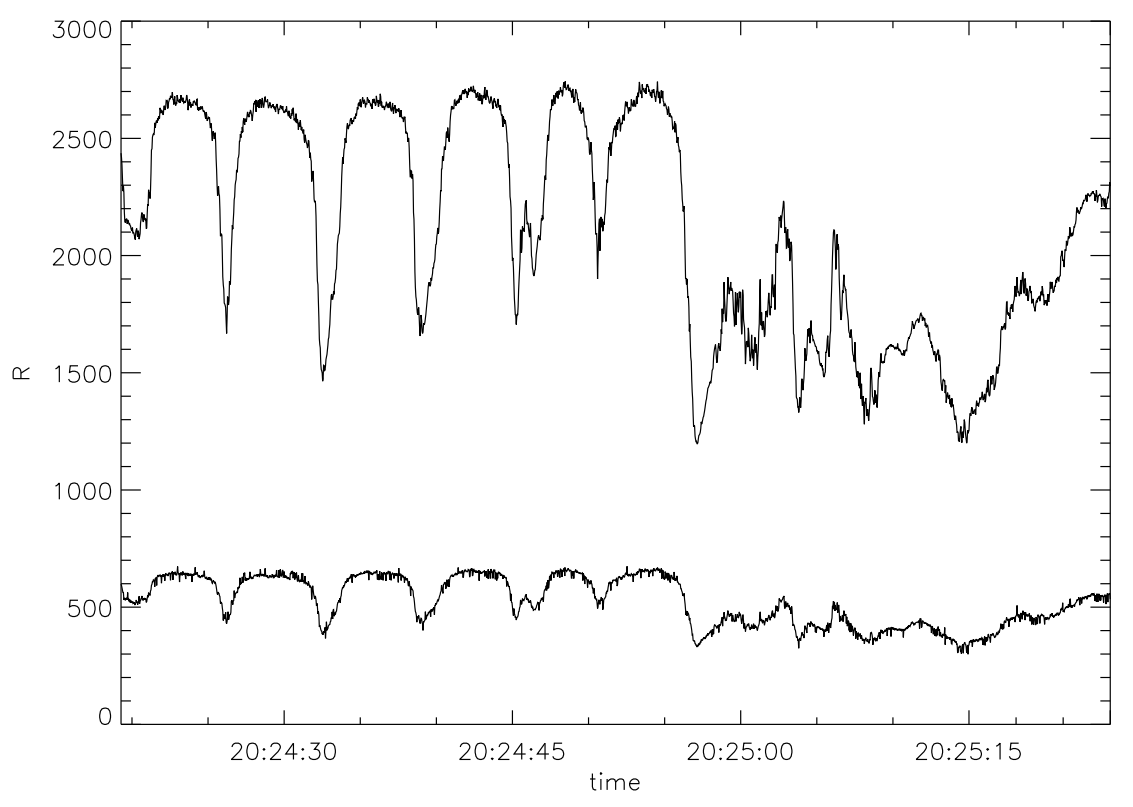

Figure 4.10: Event 3A. Mean intensity in ASK1, ASK3. 


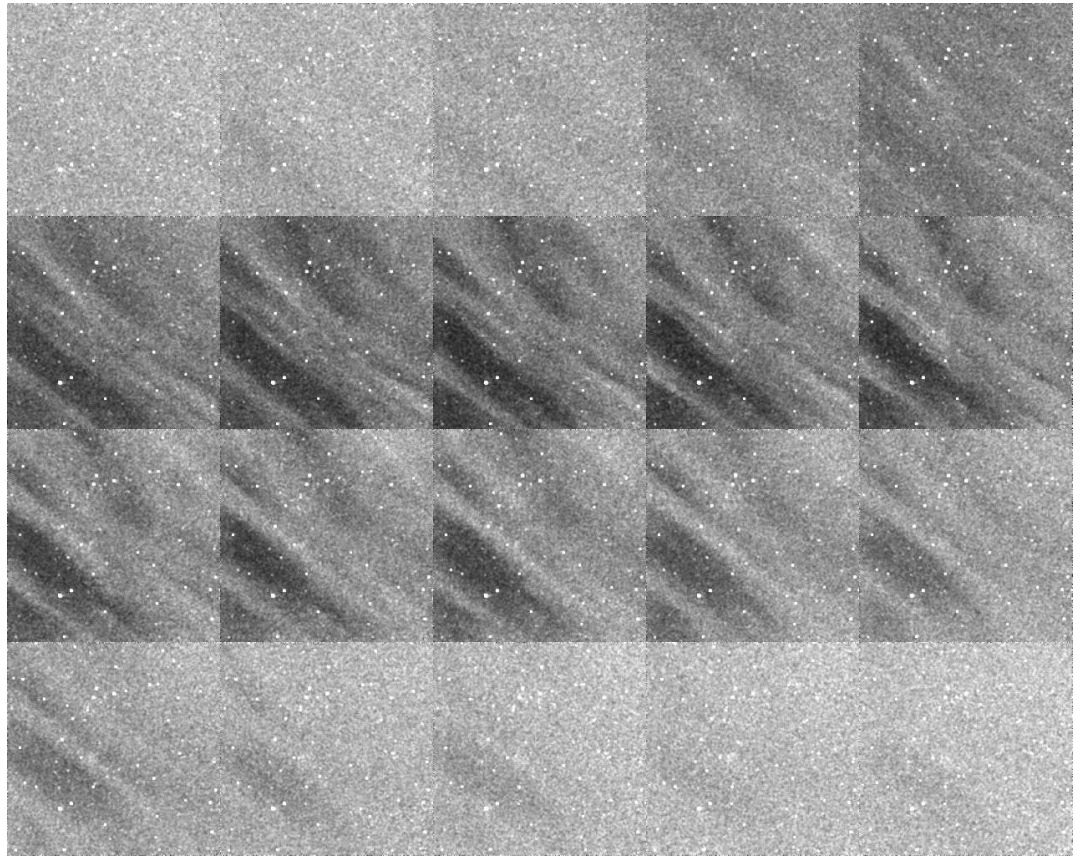

Figure 4.11: Event 3A. 20:24:31.781, $\Delta \mathrm{t}=0.125 \mathrm{~s}$.

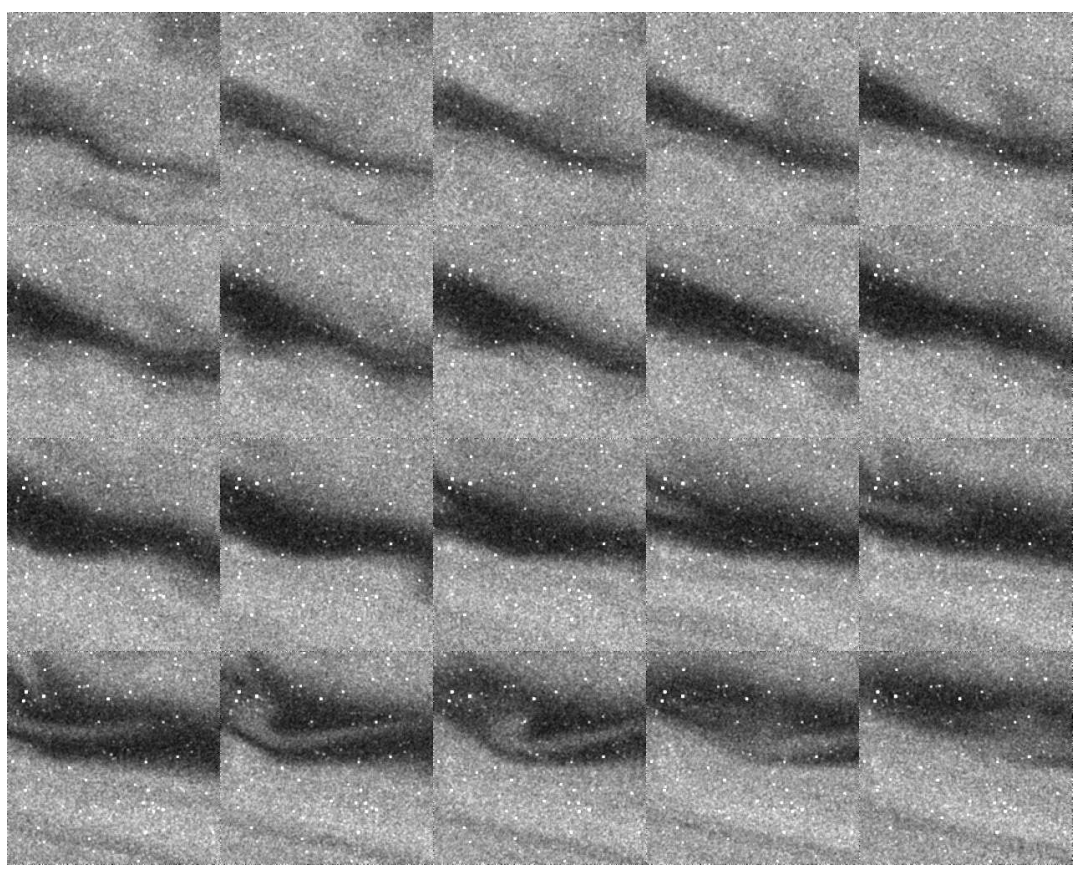

Figure 4.12: Event 3B. 20:31:44.594, $\Delta \mathrm{t}=0.469 \mathrm{~s}$. 


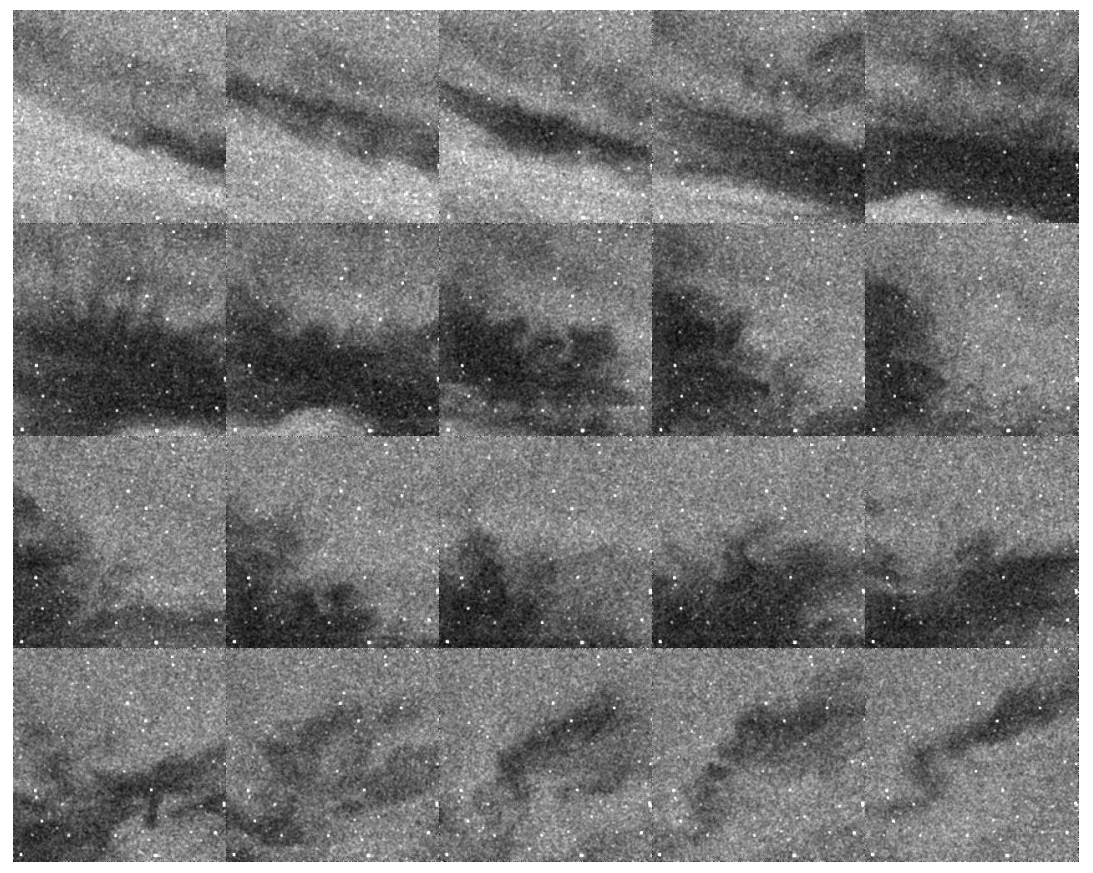

Figure 4.13: Event 4A. 21:45:57.938, $\Delta \mathrm{t}=0.781 \mathrm{~s}$.

southeast aligned arc segment which drifts towards the east. This arc segment exhibits a large degree of turbulence creating the impression of rising smoke.

Event $4 B: 21: 47$. A $1.25 \mathrm{~km} \times 5 \mathrm{~km}$ elliptical black patch drifts towards the west at approximately $2 \mathrm{~km} / \mathrm{s}$, Figure 4.14 . Its major axis is aligned roughly in the northwestsoutheast direction.

Event $4 C: 21: 47$. An irregular black arc segment enters the field of view from the north, rotating from an east-west to a north-south alignment before shifting to align with the east-west again, see Figure 4.15. The arc segment finally drifts off to the east at $1.4 \mathrm{~km} / \mathrm{s}$. There appears to be a degree of turbulence similar to that seen in Event $4 \mathrm{~A}$.

Diffuse, pulsating and black aurora, as well as some bright structured aurora, is visible in the ASK field of view until ca 00:30.

\subsection{Day 5: 7 February 2007}

A substorm begins at ca 19:00.

Event 5A: 21:10. An east-west aligned black vortex street surrounded by diffuse aurora moves towards the west, see Figure 4.16. The rotational direction of the vortices is 


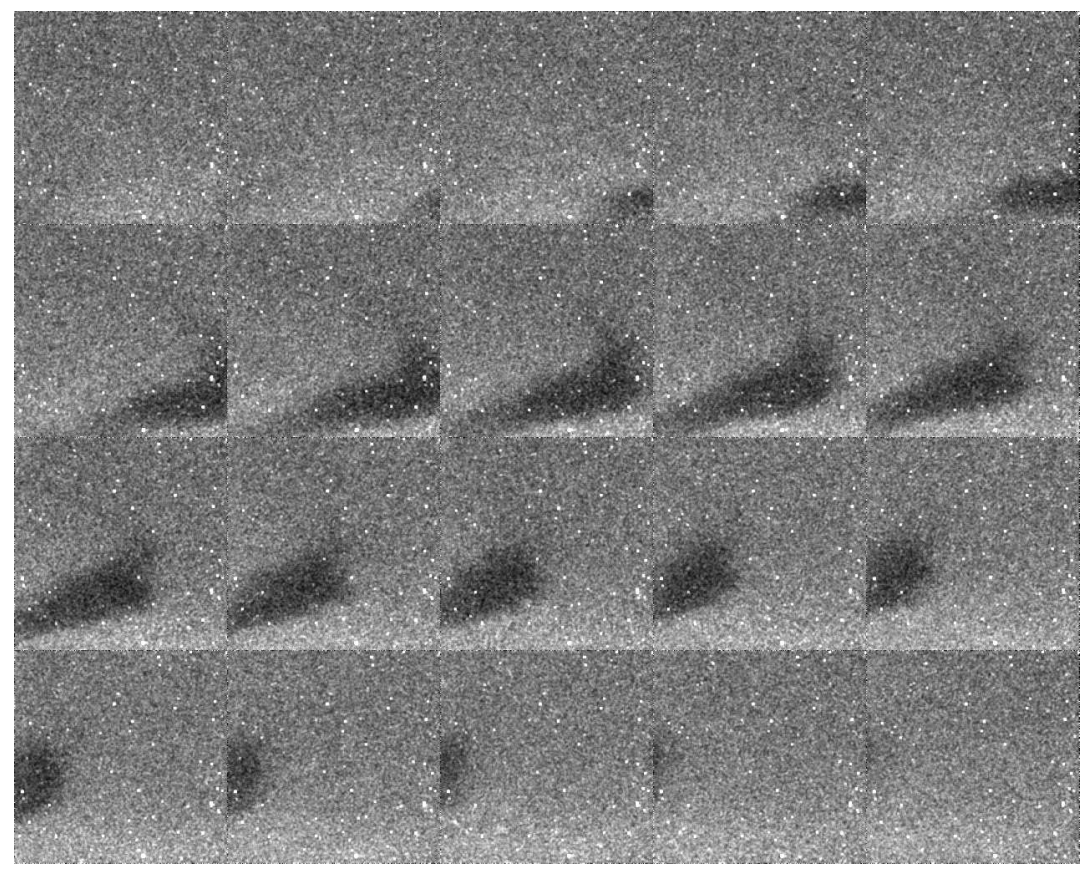

Figure 4.14: Event 4B. 21:47:36.406, $\Delta \mathrm{t}=0.188 \mathrm{~s}$.

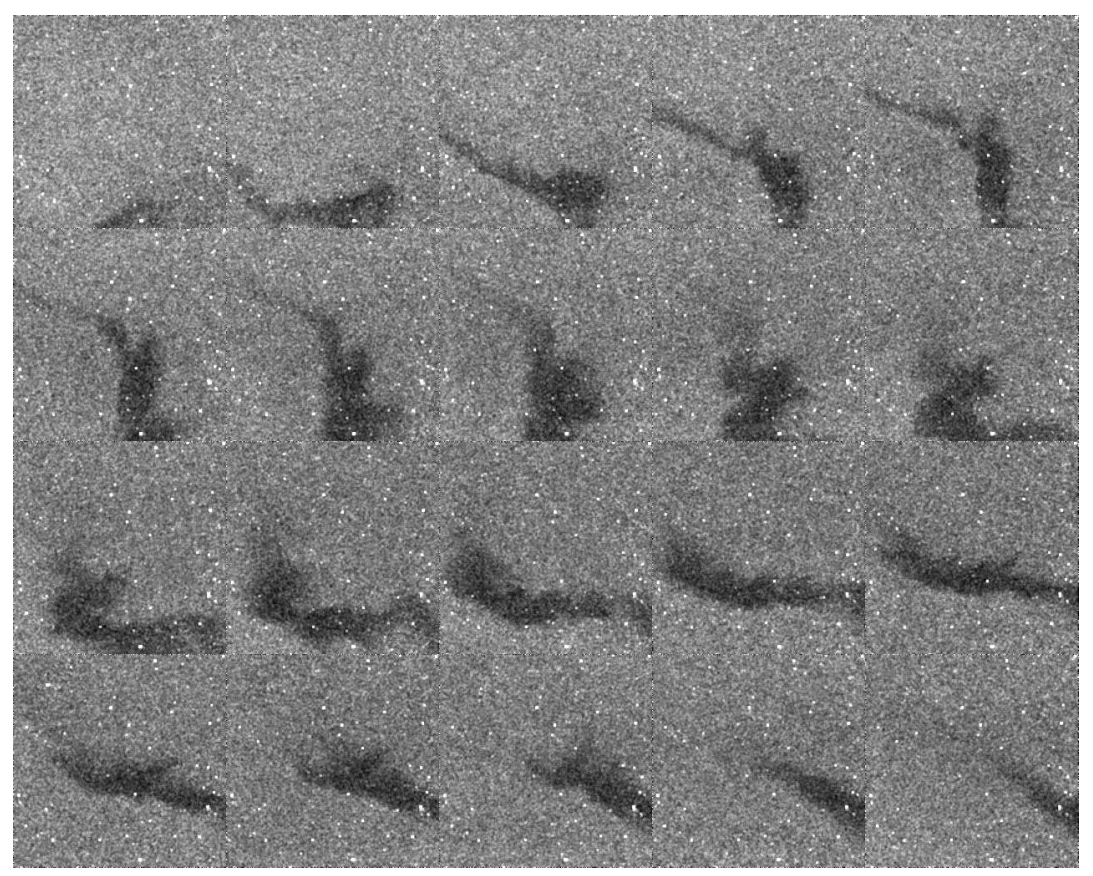

Figure 4.15: Event 4C. 21:47:44.813, $\Delta \mathrm{t}=0.625 \mathrm{~s}$. 


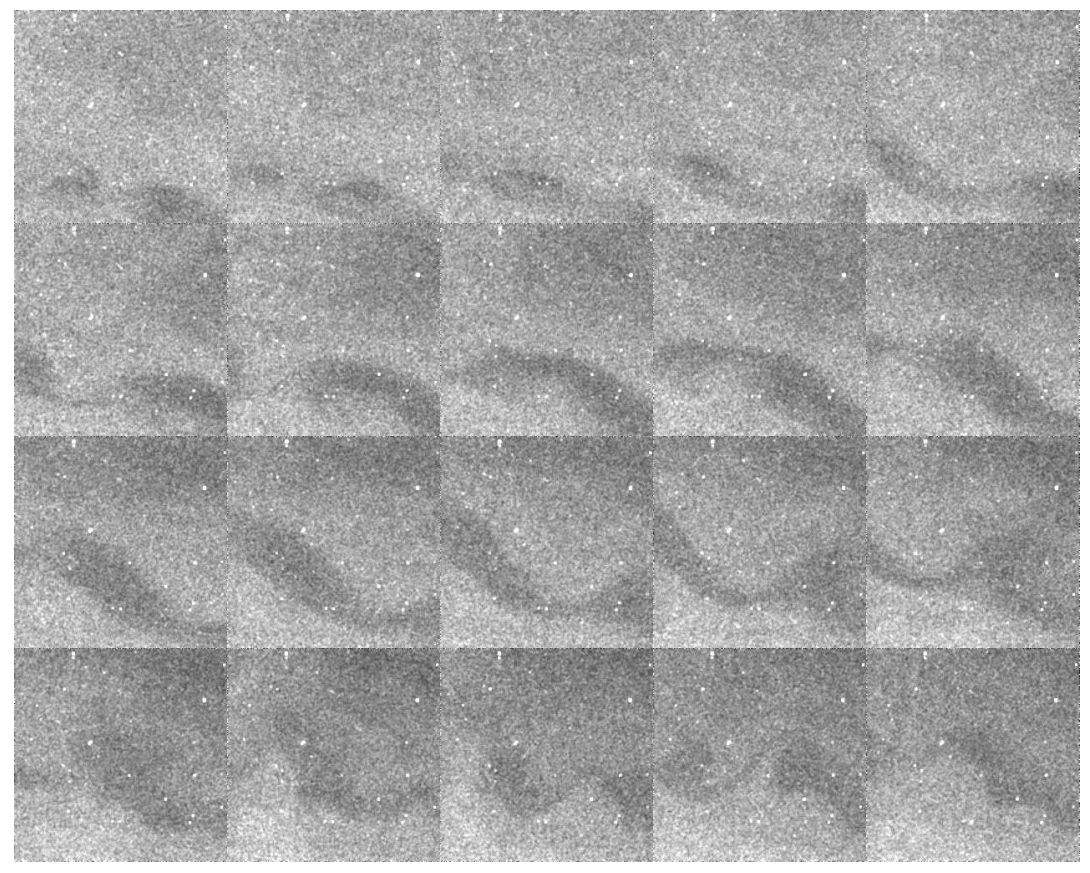

Figure 4.16: Event 5A. 21:10:26.450, $\Delta \mathrm{t}=0.500 \mathrm{~s}$.

clockwise (viewed anti-parallel to the magnetic field). The intensity of the black vortex street is approximately $10 \%$ lower than the surrounding diffuse aurora in ASK1 which means that the vortex street does not look very black; however, it is clearly a structured event which is darker than its surroundings.

A second substorm begins, with breakup occurring at 21:15. This is followed by pulsating aurora.

\subsection{Day 6: 9 March 2007}

Aurora is visible in the ASK field of view during most of the night. There is also a great deal of black aurora.

Event 6A: 22:10 - 22:11. This event does not fulfil the criteria for black aurora since multiple dynamic, discrete bright arcs are seen in the field of view, Figure 4.17. However, there are also several thin, black arc segments which separate the bright regions, some of which are no more than $200 \mathrm{~m}$ wide. These black arc segments display small ripples moving along both boundaries, in general in opposite directions.

Event 6B: 22:42 - 22:43. An east-west aligned, black arc segment exibiting small ripples splits into two, only to subsequently merge together again, see Figure 4.18 . The 
appearance is of a very elongated black ring, $40 \mathrm{~km} \times 1 \mathrm{~km}$ drifting eastwards at a velocity of $1.8 \mathrm{~km} / \mathrm{s}$. The contrast between the black ring and the surrounding diffuse aurora increases as the black ring passes across the field of view. The intensity of the front end of the ring is roughly $73 \%$ of the intensity of the surrounding diffuse aurora, while the intensity of the back end is only $47 \%$ of that of the diffuse aurora (as measured by ASK1).

Event 6C: 22:44. Three quasi-parallel, east-west aligned elongated black patches or arc segments move toward the east at approximately $2 \mathrm{~km} / \mathrm{s}$, Figure 4.19 . The middle arc segment is $24 \mathrm{~km}$ long and has a minimum width of ca $300 \mathrm{~m}$.

Event 6D: 23:05 - 23:06. A sudden surge of intensity in a region of the field of view is immediately followed by an intensity depletion in the same region. This occurs several times, although the region of interest changes. The aurora seems to be pulsating with a frequency of approximately $0.5 \mathrm{~Hz}$, Figure 4.20; however, the pulsations are localized to the dark regions which resemble elongated black patches or arc segments aligned roughly in the northwest-southeast direction, Figure 4.21.

Event 6E: 23:07 - 23:08. A northwest-southeast aligned black arc folds back on itself to create a 2 -shaped black structure with a very sharp bend, see Figure 4.22. The base of the 2 is ca $300 \mathrm{~m}$ wide. The 2 -shaped structure subsequently unbends transforming into a wavy black arc, and is joined by a second quasi-parallel wavy black arc situated to the southeast of the first. The two black arcs drift northward.

The aurora begins to pulsate at ca 23:30. This is followed by more black aurora after midnight, and also more pulsating aurora. 


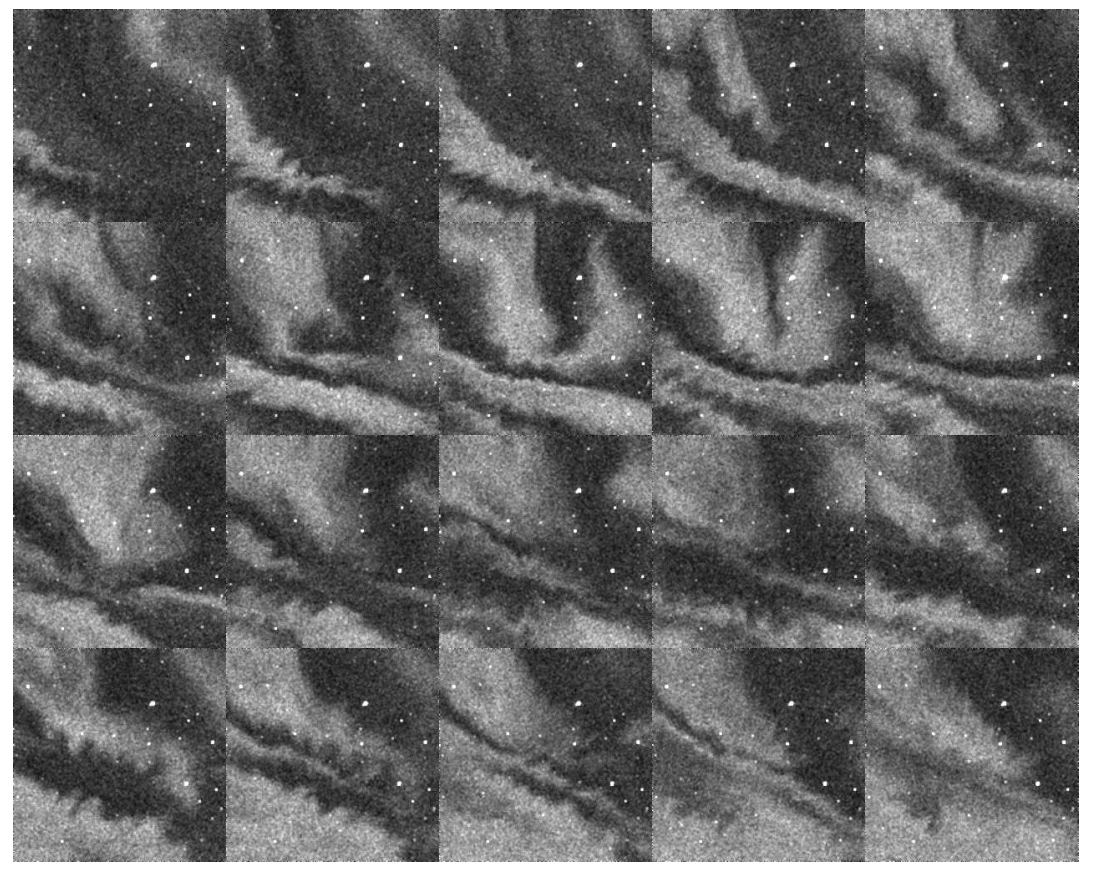

Figure 4.17: Event 6A. 22:10:40.950, $\Delta \mathrm{t}=0.750 \mathrm{~s}$.

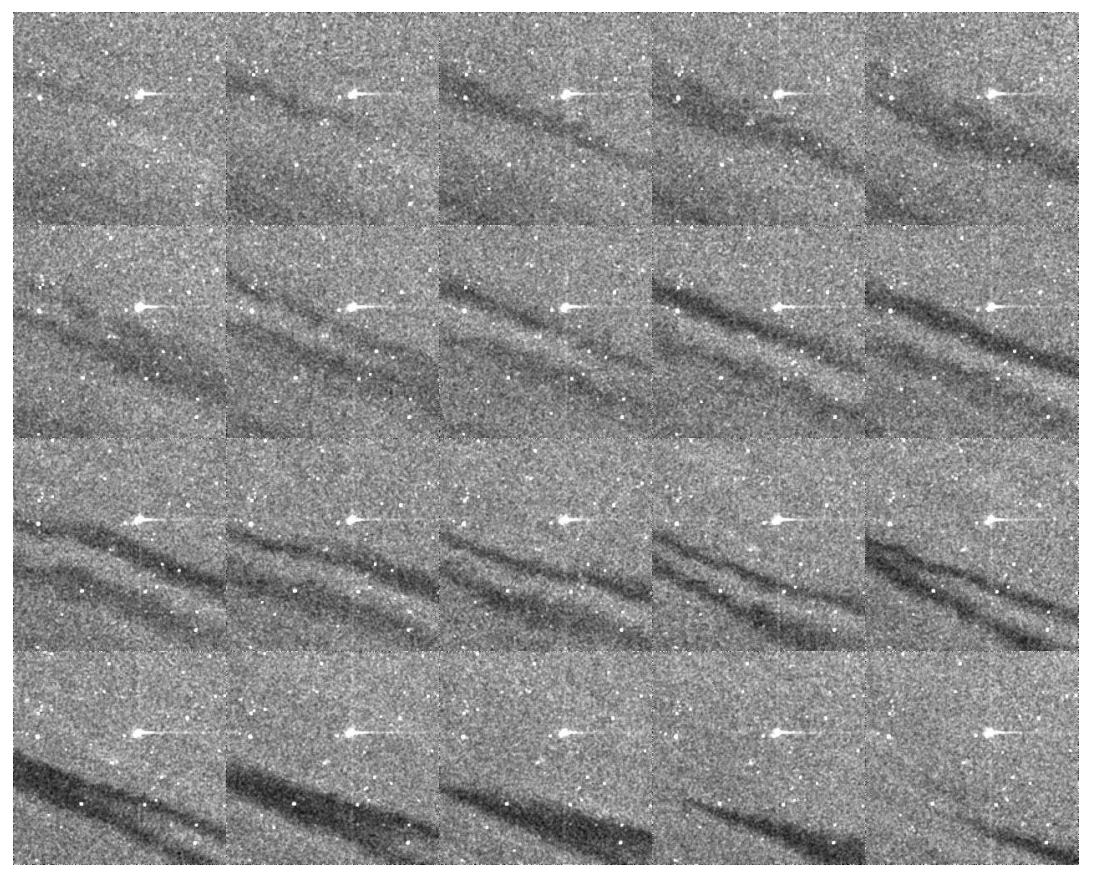

Figure 4.18: Event 6B. 22:42:48.950, $\Delta \mathrm{t}=1.00 \mathrm{~s}$. 


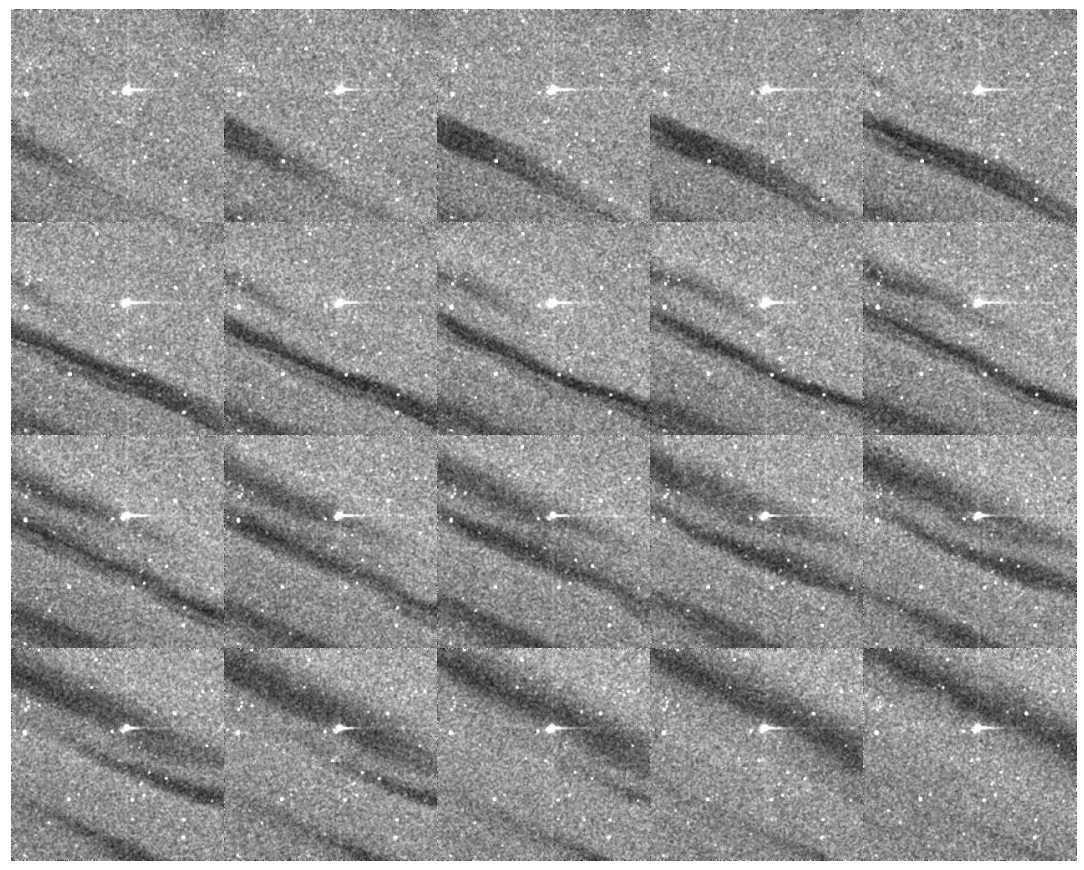

Figure 4.19: Event 6C. 22:44:01.450, $\Delta \mathrm{t}=0.600 \mathrm{~s}$.

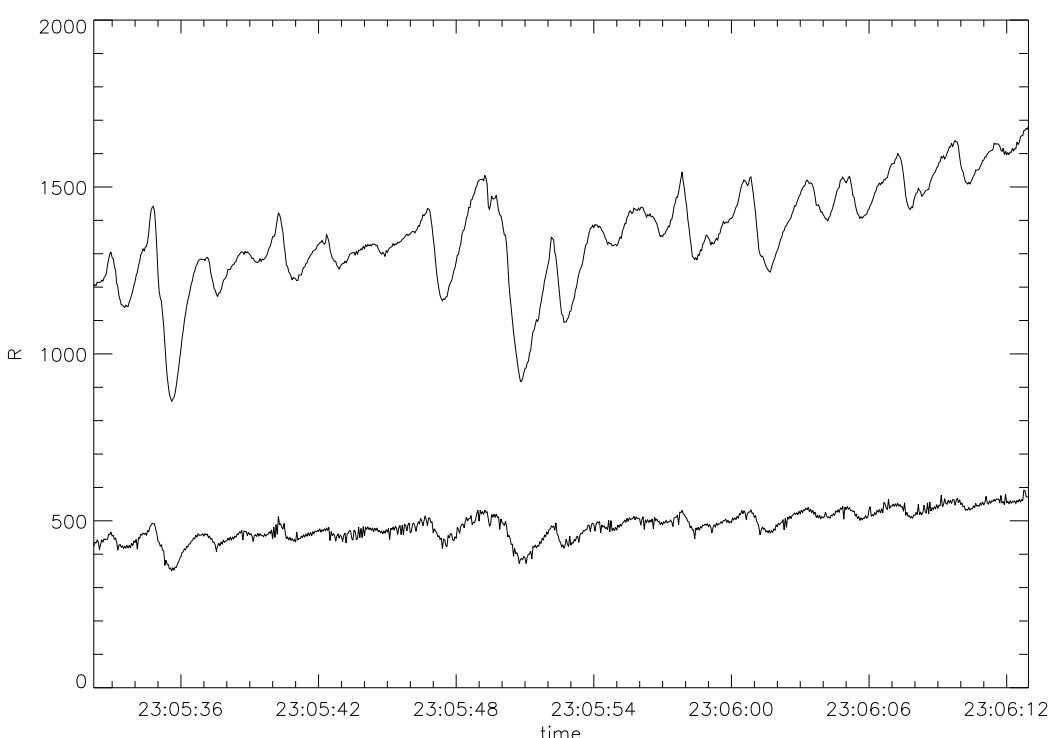

Figure 4.20: Event 6D. Mean intensity in ASK1, ASK3. 


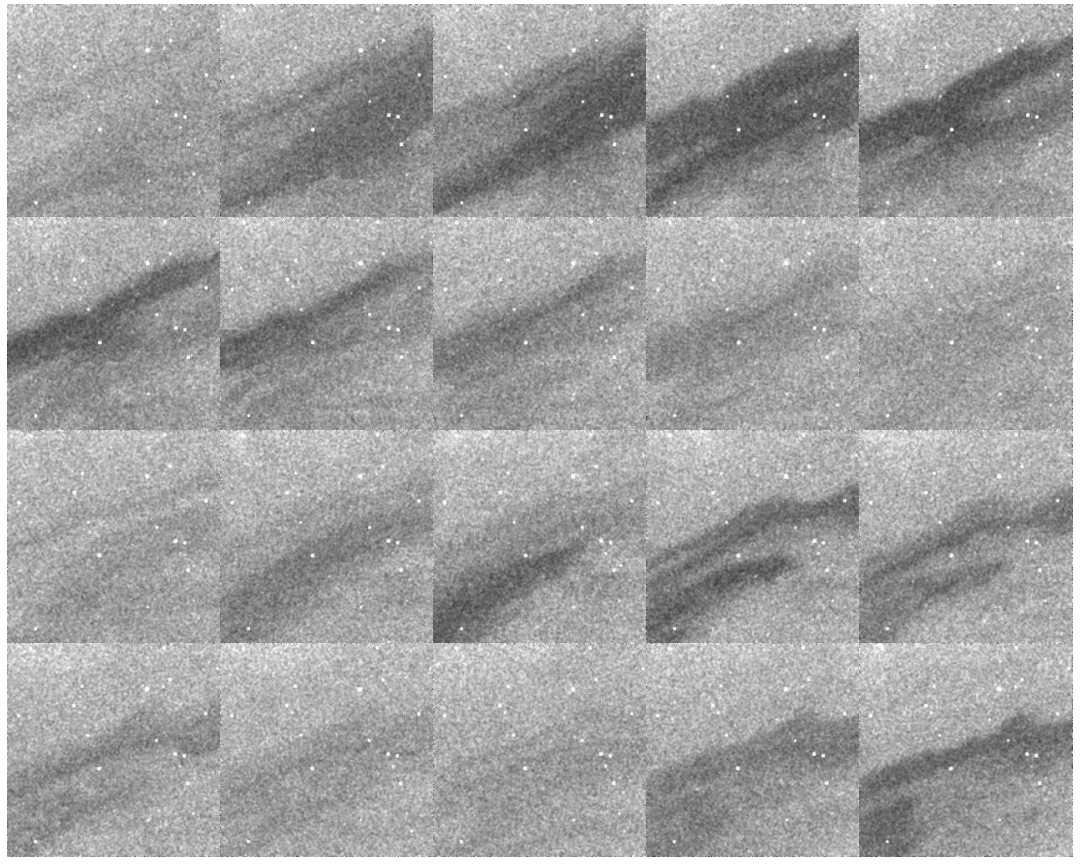

Figure 4.21: Event 6D. 23:06:00.950, $\Delta \mathrm{t}=0.250 \mathrm{~s}$.

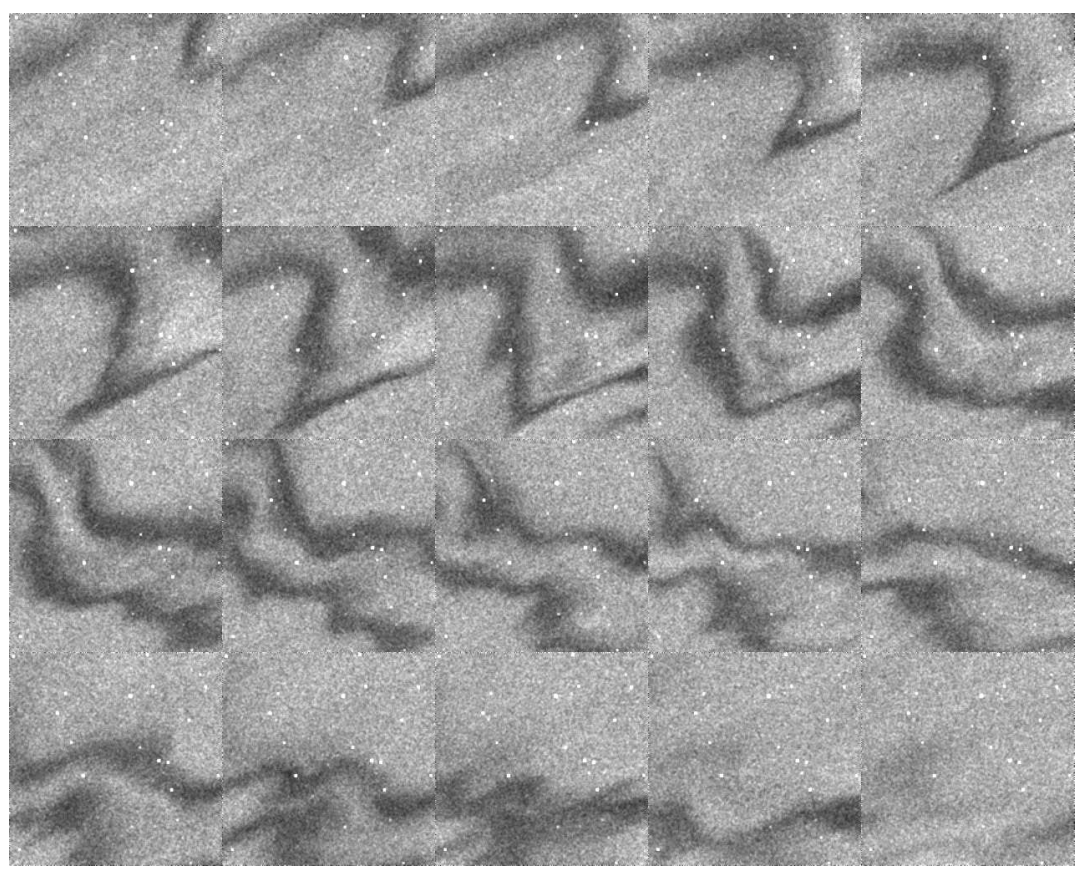

Figure 4.22: Event 6E. 23:07:43.700, $\Delta \mathrm{t}=1.25 \mathrm{~s}$. 


\section{Chapter 5}

\section{Analysis}

\subsection{The Auroral Signal}

One of the most important aims of this study was to ascertain whether emission intensity levels fall to zero in regions of black aurora. Therefore, an attempt was made to determine the absolute intensity of the various auroral events.

\subsubsection{Darks and flats}

ASK records images at the desired frame rate during 20-minute time intervals. Immediately preceding each 20 minute long image sequence, a short series of dark images is recorded with the shutter closed. These frames are averaged to create a dark field, dark, which is subtracted from the succeeding raw images. The images also need to be corrected with regard to variations in pixel sensitivity. Flat field images, flat, are created by averaging over a sequence of frames showing total cloud cover.

The measured signal, raw, is made up of signal counts, S, bias counts, B, and dark counts, D:

$$
r a w=S+B+D
$$

with

$$
\text { dark }=B+D
$$

and

$$
\text { flat }=S_{\text {flat }}+B+D
$$

The corrected signal, $\mathrm{S}$, is determined from the measured image, raw, as follows:

$$
S=\frac{r a w-d a r k}{\text { flat }- \text { dark }}
$$

$\mathrm{S}$ is expressed as the number of counts detected by each pixel $\times$ frame rate. To provide a quantitative measurement of the absolute intensity, $\mathrm{S}$ must be transformed 
into Rayleighs. The Rayleigh is a unit of surface brightness, defined as the brightness of the column-integrated emission rate of $10^{6}$ photons $/ \mathrm{cm}^{2} / \mathrm{s}$ :

$$
1 \text { Rayleigh }=\frac{10^{6}}{4 \pi} \text { photons } / \mathrm{cm}^{2} / \mathrm{s} / \mathrm{srad}
$$

The intensity calibration is accomplished by comparing the apparent brightness of star signals detected by ASK to the theoretical spectral flux of the stars recorded in e.g. the SAO star catalogue.

\subsubsection{Background removal}

After the raw ASK images have been dark field and flat field corrected, there still remains a background emission emanating from sources such as the moon and clouds. Another factor to be considered is the bias drift that arises when the temperature of the ASK imagers is non-constant. In this case, the number of bias counts, B, (see Eq. (5.2)) varies with time. When determining the dark field, dark, at a certain time the change in B must be taken into account:

$$
\text { dark }=B+\Delta B+D
$$

where $\mathrm{B}$ is the number of bias counts at the time that the dark images were recorded, and $\Delta \mathrm{B}$ is the change in the number of bias counts at the time that the image of interest was acquired.

Intensity plots over sequences of images lacking aurora showed that the bias drift was approximately linear for both ASK1 and ASK3. Therefore, it seemed reasonable to approximate the bias drift as a straight line. The dark field following an auroral event, dark2, was subtracted from the dark field preceding it, dark1:

$$
\Delta B \approx \frac{\text { frame number }}{\text { number of frames }} \times(\text { dark } 1-\text { dark } 2)
$$

The mean value, drift, of the $256 \times 256$ elements of $\Delta \mathrm{B}$ was calculated to be used in the determination of the absolute intensity.

The background intensity of each event was calculated from sequences of images without aurora. Two factors taken into consideration when choosing images for background determination were the following:

1. The moon should be in the same phase and in the same position during the background sequence as during the auroral event.

2. A background sequence should be found for which the bias drift was as close to linear as possible.

Once a suitable series of images had been found, the bias drift during the background sequence was calculated and added to each image in the sequence, and the mean intensity of each image was determined. Finally, the mean intensities of the background sequence were averaged to obtain a value for the background intensity in counts $\times$ frame rate/pixel. 

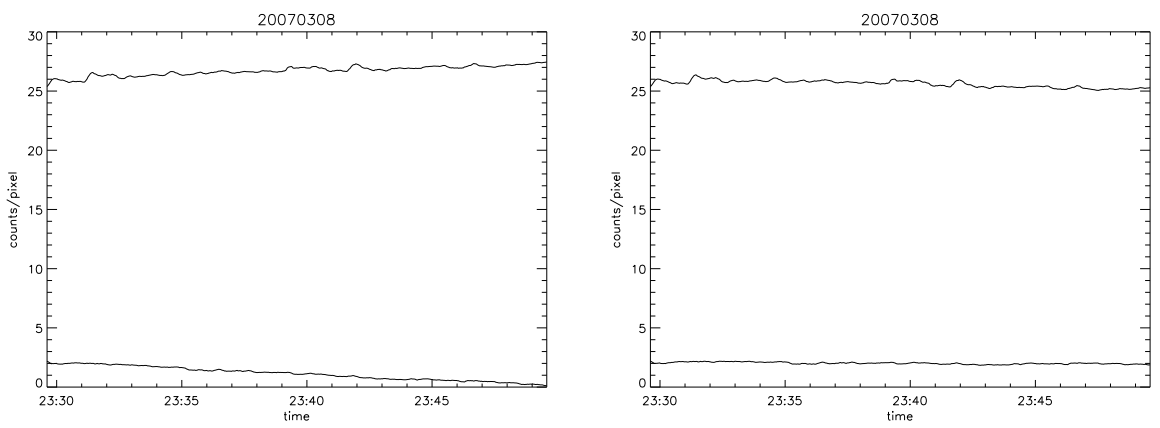

Figure 5.1: Background intensity before (left) and after (right) bias drift removal.

An example is given in Figure 5.1 which shows the background sequence chosen for Days 5 and 6. The mean intensity of each ASK1 and ASK3 image prior to bias drift removal is shown in the left plot. The bias drift is seen to be approximately linear for both cameras. Once the bias drift has been removed, right plot, the mean intensity for the whole sequence of frames can be determined. The background intensities for Days 5 and 6 were thus determined to be 25.6 counts $\times$ frame rate/pixel for ASK 1 and 2.0 counts $\times$ frame rate/pixel for ASK3.

The background value was subsequently subtracted from each image in the auroral event sequence, and the bias drift which occurred during the auroral sequence was added:

$$
\text { corrected image }=\text { image }- \text { background }+ \text { drift }
$$

The corrected image was then multiplied by a calibration factor to obtain the intensity of the event expressed in Rayleighs.

\subsubsection{Noise reduction}

The ASK images were impaired by two major types of noise. Stars generate very high signals, particularly in ASK1, which disturb the determination of the much lower auroral signal. General random noise was also a problem, especially in ASK3, since the recorded signal was often very low, typically only $20-25 \%$ of that recorded by ASK1.

Two different filters were used to reduce noise, a median filter and a Lee filter. Both filters are ready made functions found in the IDL library. Median filtering is effective in removing isolated high and low values such as star signals, while the Lee filter algorithm removes additive image noise.

The top two graphs in Figure 5.2 show the mean intensity in ASK1 (left) and ASK3 (right) of a vertical slice of width 10 pixels centred at xpixel=165 through image 9 , Event 3B (see Figure 4.12). The median filter has been used for the middle two graphs which show that signals from stars, as well as other isolated high and low values, have been reduced considerably. Finally, for the bottom two graphs, the Lee filter has been added to reduce random noise. 

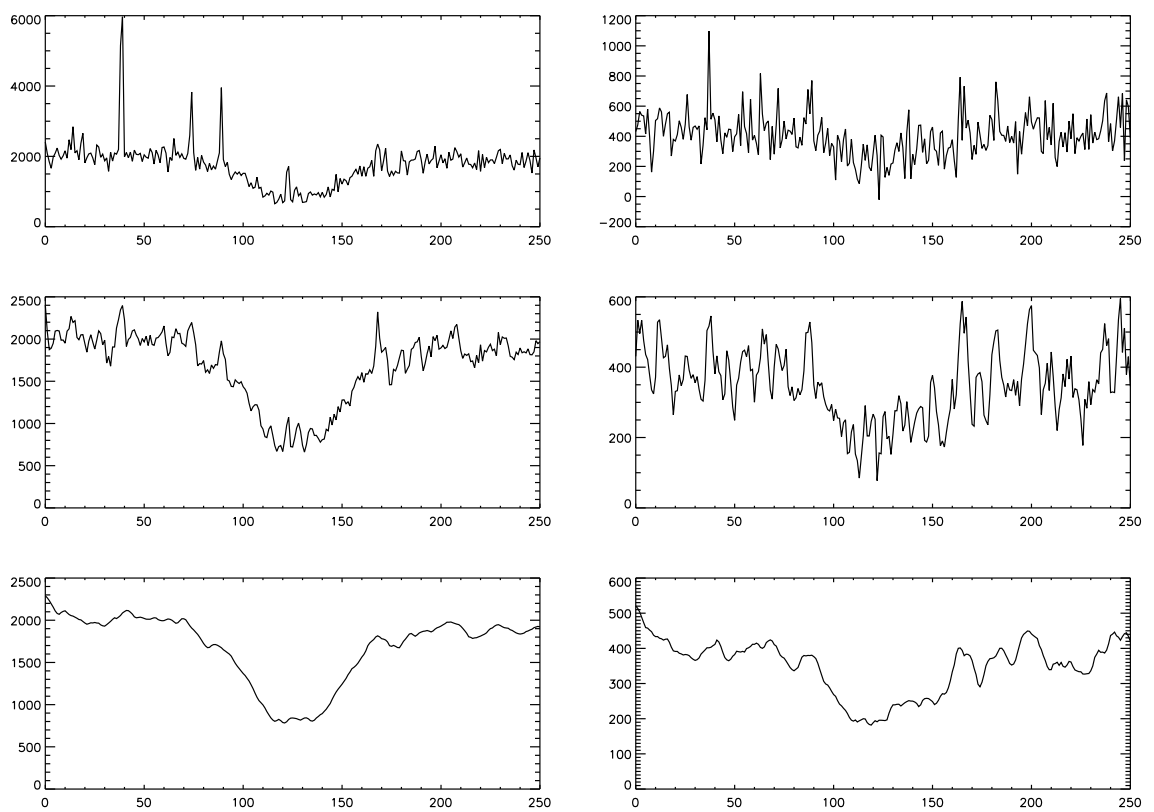

Figure 5.2: Intensity (in Rayleighs) of vertical slice at xpixel=165, Event 3B, image 9.

\subsection{Determining the Absolute Intensity}

Once background and noise have been deducted from the images, it is possible to estimate the absolute intensity of the black aurora in ASK1 and ASK3. This is a challenge since the black aurora is dynamic, changing in shape and intensity while simultaneously drifting across the field of view.

Figure 5.3 and Figure 5.4 show the mean intensity in ASK 1 and ASK 3 of a vertical slice of width 10 pixels centred at xpixel $=128$ for a series of images from Event $3 \mathrm{~B}$ (Figure 4.12). The plots give a cross sectional view of the black arc, which is seen to broaden, grow blacker and drift towards the south as time passes.

One method of determining a rough estimate of the intensity of a black auroral event is to define the black structure seen in each of a series of images as a region of interest. It is then possible to calculate the mean intensity within the black region for each frame to obtain a measurement of how much the intensity actually decreases relative to the surrounding diffuse aurora, see Table 6.2.

\subsection{The Intensity Ratio}

ASK1 detects $\mathrm{N}_{2}$ emissions originating from high-energy electron precipitation, ASK3 detects OI emissions which are mainly produced by low-energy electron precipitation. The intensity ratio between these low and high energy emissions, $\mathrm{OI} / \mathrm{N}_{2}$, can be used 

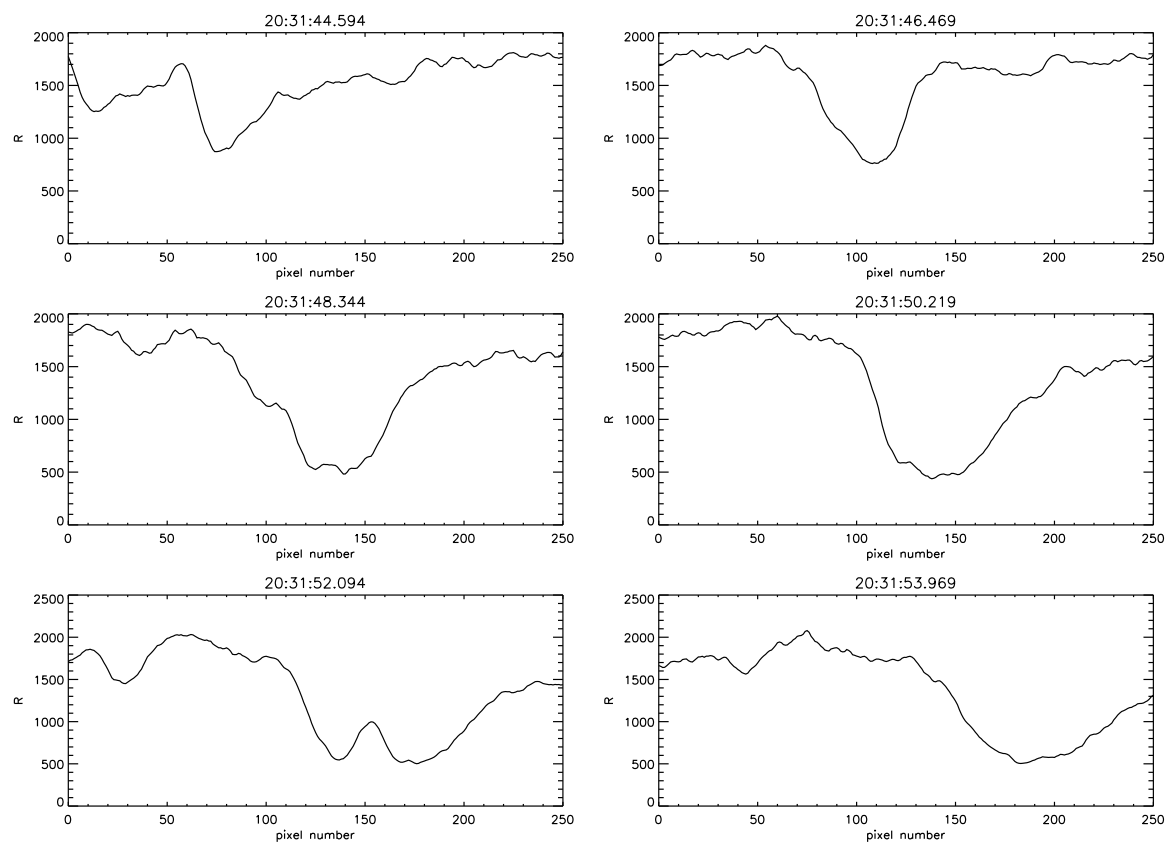

Figure 5.3: Intensity in ASK1 of vertical slice at xpixel=128, Event 3B.
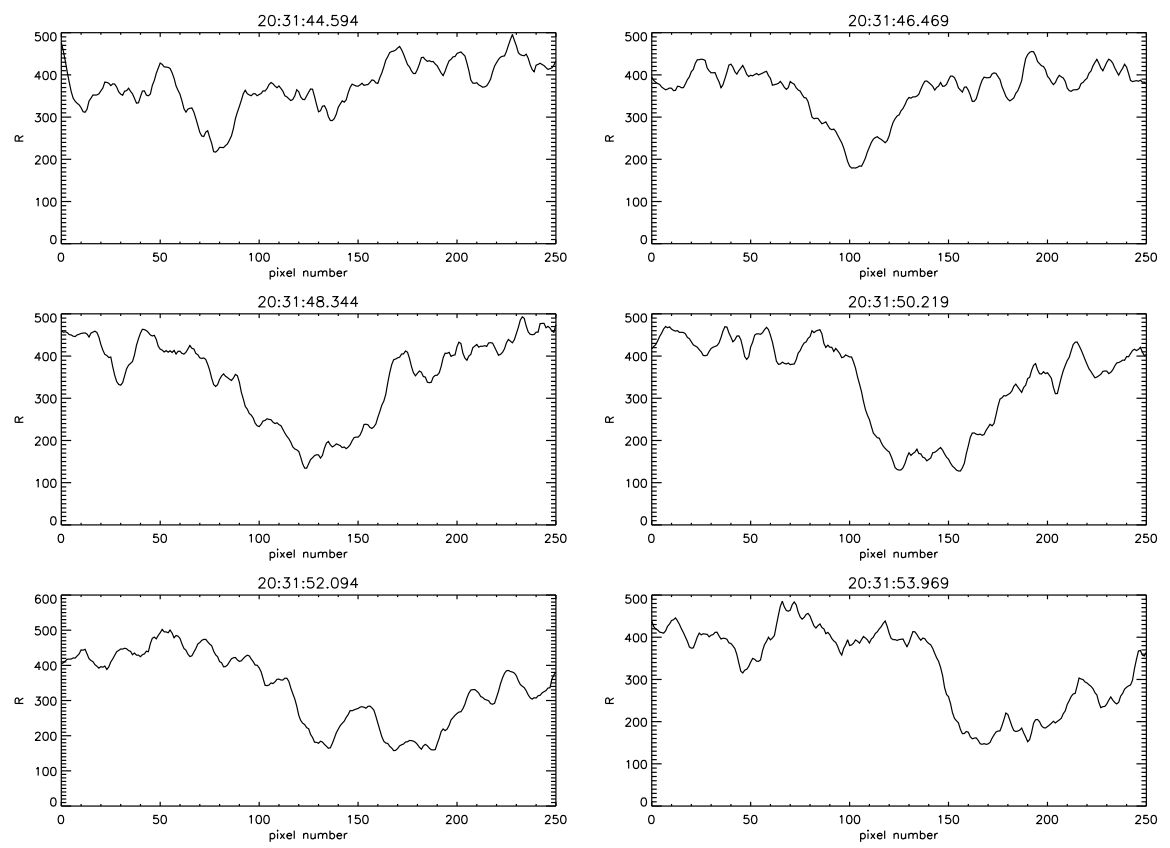

Figure 5.4: Intensity in ASK3 of vertical slice at xpixel=128, Event 3B. 

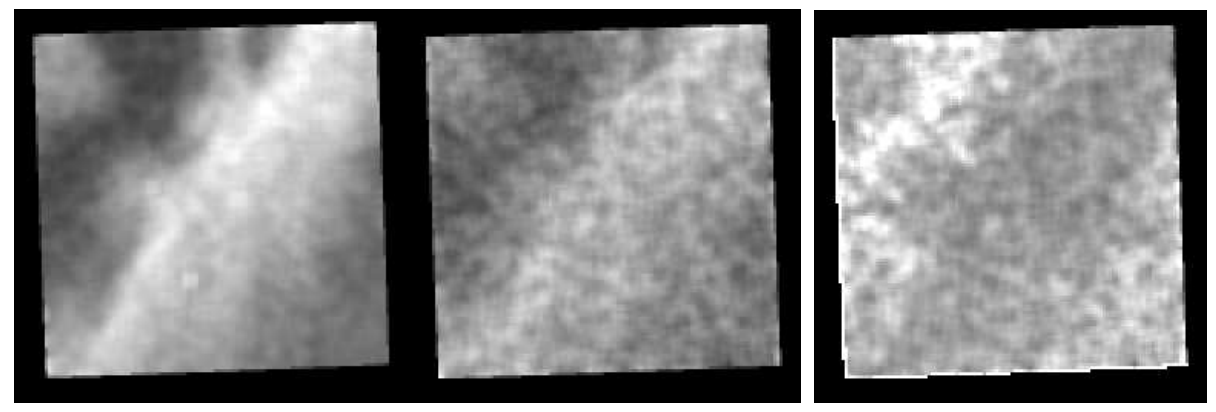

Figure 5.5: Event 2A. Simultaneous images ASK1, ASK3, ASK3/ASK1 intensity ratio.

to determine whether the relative decrease in flux of low-energy electrons differs from that of high-energy electrons within regions of black aurora.

An analysis of the intensity ratio is only applicable when the morphology of an auroral event is the same in ASK1 and ASK3. Identical morphology indicates that the altitude of both emissions is the same. This was found to be the case for all the studied events.

Figure 5.5 shows simultaneous images from ASK1 and ASK3 together with the ratio of the images ASK3/ASK1. The appearance of the aurora is the same through both filters, although clearer in ASK1 due to the much higher intensity, see Table 4.1.

The OI/ $\mathrm{N}_{2}$ emission intensity ratio is not uniform. The dark areas in the ASK1 and ASK3 images show up as white areas in the ratio plot. This means that the $\mathrm{N}_{2}$ emissions decrease more than the OI emissions in the dark regions. If, on the other hand, the ratio plot had been uniformly grey, then the dark regions would be due to an overall decrease in particle flux; the relative decrease in high and low energy electron precipitation would be equal, and the ratio would be the same for the whole field of view.

\subsection{Ion chemistry model}

The Southampton ion chemistry model [Lanchester et al., 2001] performed a numerical estimation of the energy of the precipitating electrons using the $\mathrm{OI} / \mathrm{N}_{2}$ ratios obtained from the optical data. Very briefly, this model encompasses all the major ionospheric interactions, e.g. reaction rates, recombination, etc. Various activity parameters are used as input to achieve the best possible representation of the ionosphere at a particular time. The energy distribution input can be modelled as a Gaussian (i.e. monoenergetic), Maxwellian, power-law, or as a combination of the three.

Only the simplest mono-energetic energy distribution was used as input to the model in this study. The OI/ $\mathrm{N}_{2}$ emission intensity ratios for the black structures, as well as for the surrounding diffuse aurora, were compared to the emission rates obtained from the model. In this way, a numerical value of the characteristic energies of the different auroral regions can be estimated. The goal was to determine whether the 
energy of the precipitating electrons differed within the black regions compared to the bright aurora.

\subsection{Comparison of optical and radar data}

EISCAT radar data were available for Day 1, 17 October 2006 and Day 2, 22 October 2006. The EISCAT ionospheric electron density measurements were compared to the in-beam emission intensities recorded by ASK, with the aim of determining whether the ionospheric electron density was depleted when black structures crossed the radar beam.

The time resolution of the EISCAT electron density data is $4 \mathrm{~s}$, which is much lower than that of the optical data. However, when the electron temperature is constant, i.e. $\mathrm{T}_{e}=\mathrm{T}_{i}=$ constant, the power profile (time resolution $=0.5 \mathrm{~s}$ ) can be used as a measure of the electron density. 


\section{Chapter 6}

\section{Results and Discussion}

\subsection{Summary of the Observed Events}

Sixteen events were selected for analysis from data recorded during the 2006/2007 observational campaign in Ramfjordmoen (Troms $\varnothing$ ), Norway (geomagnetic latitude, $69^{\circ} \mathrm{N}$ ). All events, with the exception of Event $2 \mathrm{~A}$, occurred during the time period lasting from ca $1 \mathrm{~h}$ before to $1.5 \mathrm{~h}$ after local magnetic midnight.

No black auroral events were found in the observational data from Svalbard (geomagnetic latitude, $78^{\circ} \mathrm{N}$ ). This is probably due to the high latitude, where dayside aurora and phenomena originating in the plasma sheet boundary layer prevale. Auroral phenomena occurring at Troms $\varnothing$ latitudes are more likely to originate in the central plasma sheet.

The sixteen events can be divided into three groups: I) unsheared black aurora, II) sheared black aurora, and III) dark regions adjacent to discrete bright aurora. The events in group III) do not qualify as black aurora, according to the definition. The events in group I) can be divided into two sub-groups: Ia) unsheared black aurora without pulsations, and $\mathrm{Ib}$ ) unsheared black aurora with pulsations.

It was impossible to determine whether the dark structure in Event 2D was a proper black arc or only the dark space between two bright arcs. It also seemed to briefly exhibit a small degree of shear which did not, however, develop into full-blown curls. Therefore, Event 2D constitutes a limiting case both with regard to shear structure and the definition of black aurora.

Table 6.1 summarizes scale sizes and other characteristics of the events, assuming an altitude of $100 \mathrm{~km}$. Widths marked with a star denote the widths of multiple structures, while unmarked widths are those of single structures. The widths of black structures often change with regard to time and space. Velocities marked with a star denote the speeds of curls, ripples and waves along black arcs. The unmarked velocities are the drift speeds of black structures.

Previously observed black structures have widths ranging between $100 \mathrm{~m}$ and $5 \mathrm{~km}$ [Peticolas et al., 2002]. The widths of the black structures observed in this study range from $100 \mathrm{~m}$ to $3 \mathrm{~km}$, thereby falling into the most fine-scaled category. The narrow 


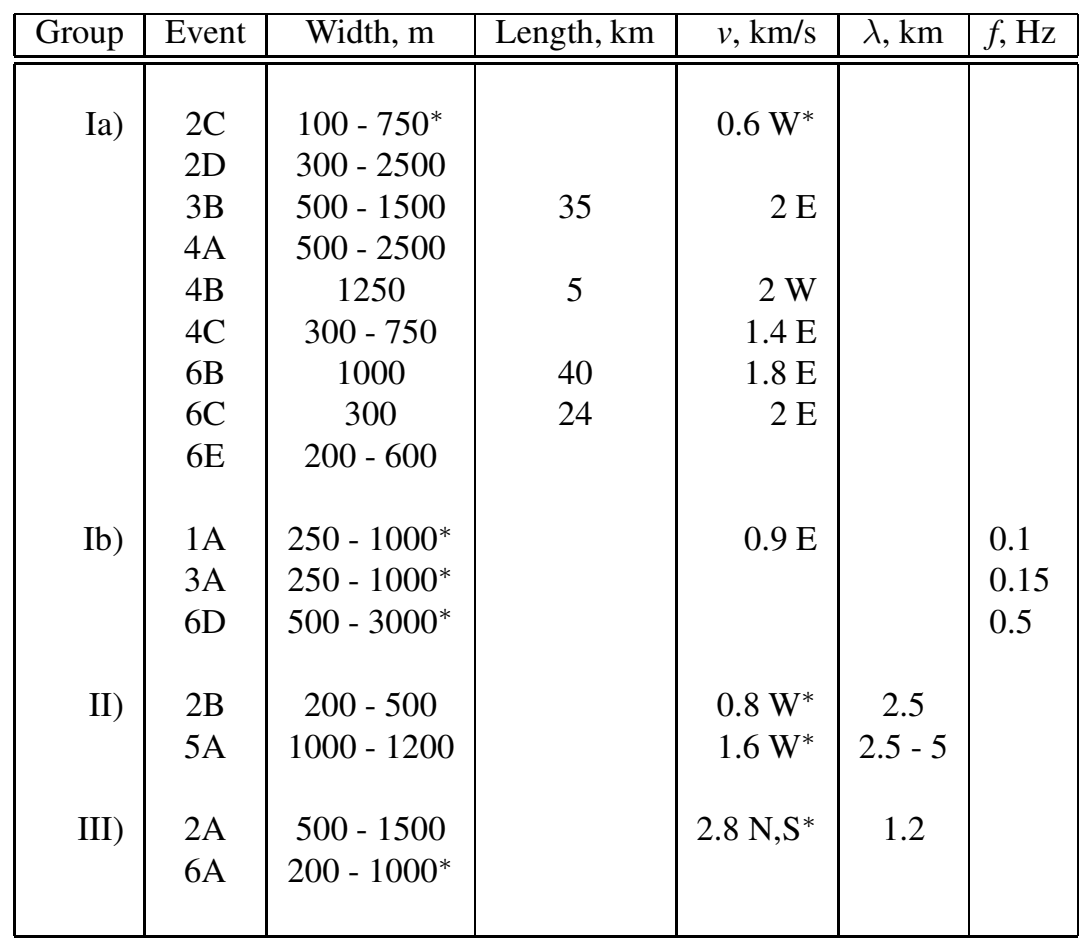

Table 6.1: Event characteristics. Ia) unsheared black aurora without pulsations, Ib) unsheared black aurora with pulsations, II) sheared black aurora, and III) dark regions adjacent to discrete bright aurora. 


\begin{tabular}{|l|c|c|c|c|c|}
\hline & & \multicolumn{2}{|c|}{ Mean Intensity, R } & \multicolumn{2}{c|}{ Relative Reduction, \% } \\
Event & Time, UT & ASK1 & ASK3 & ASK1 & ASK3 \\
\hline \hline & & & & & \\
2B & $20: 50: 58-20: 51: 04$ & 370 & 130 & 40 & 24 \\
2C & $20: 52: 08-20: 52: 14$ & 420 & 140 & 29 & 16 \\
2D & $20: 54: 44-20: 54: 50$ & 420 & 130 & 44 & 31 \\
& & & & & \\
3B & $20: 31: 46-20: 31: 53$ & 650 & 210 & 60 & 47 \\
& & & & & \\
4A & $21: 46: 09-21: 46: 15$ & 1000 & 260 & 30 & 20 \\
4B & $21: 47: 37-21: 47: 38$ & 740 & 230 & 30 & 9 \\
4C & $21: 47: 46-21: 47: 49$ & 790 & 230 & 34 & 24 \\
& & & & & \\
5A & $21: 10: 29-21: 10: 34$ & 1270 & 420 & 10 & 8 \\
& & & & & \\
6B & $22: 42: 59-22: 43: 04$ & 400 & 140 & 37 & 28 \\
6C & $22: 44: 16-22: 44: 21$ & 460 & 160 & 35 & 24 \\
6E & $23: 07: 47-23: 07: 52$ & 900 & 380 & 35 & 22 \\
& & & & & \\
\hline
\end{tabular}

Table 6.2: Relative reduction of intensity in regions of black aurora.

field of view of the ASK imager imposes an upward limitation on the scale sizes of the observed black aurora.

It was possible to measure the lengths/major axis of three black arcs or arc segments, and one black patch. Two of these structures are intermediate between black arc segments, 20 - $30 \mathrm{~km}$, and black arcs, $>40 \mathrm{~km}$. The structure observed in Event 6B constitutes quasi-parallel, double arc segments which join together at both ends, giving the appearance of a very long black ring.

The drift speeds of six black structures were measured. The average speed was determined to be $1.7 \mathrm{~km} / \mathrm{s}$. Five of the black structures have an eastward drift, which is in agreement with previous observations. However, the black patch observed in Event 4B drifts towards the west, which is atypical.

Curls and waves were observed to travel toward the west. Curl wavelengths correspond with previous measurements.

\subsection{Absolute Intensity of Black Aurora}

Table 6.2 shows the mean intensities of $\mathrm{N}_{2}$ (ASK1) and OI (ASK3) emissions within a black structure during a short time interval for the events in groups Ia) and II). The mean $\mathrm{N}_{2}$ and $\mathrm{OI}$ emission intensities were also determined for the surrounding diffuse aurora. The relative reduction indicates the decrease in intensity of the black structure relative to the diffuse aurora.

In general, it was only possible to determine the mean intensities within black re- 
gions during very short time periods, since the intensities of the black structures varied with time and space. The dark regions of the events in group III) were surrounded by dynamic, bright aurora; therefore, it was not possible to determine the relative reduction.

The results of the measurements show that emission intensity levels do not fall to zero within regions of black aurora. The absolute intensities of the black structures range between 370 and 1270 Rayleighs in ASK1, and 130 and 420 Rayleighs in ASK3. For most of the events, the $\mathrm{N}_{2}$ emission intensity is $30-44 \%$ lower in the black structures compared to the surrounding diffuse aurora. OI emissions are, in general, 16 - $31 \%$ lower within the black aurora than the diffuse aurora.

The mean relative reduction in the $\mathrm{N}_{2}$ emission intensity is determined to be $35 \%$, while the mean relative reduction in the OI emission intensity is $23 \%$. All of the measured events exhibit a greater decrease of $\mathrm{N}_{2}$ emissions than of $\mathrm{OI}$ emissions in regions of black aurora. This is in agreement with Gustavsson et al. (2008) who measured relative intensity reductions of $25 \%$ at $4278 \AA$ (high-energy electron precipitation), and $18 \%$ at $8446 \AA$ (low-energy electron precipitation).

The relative decrease in OI emission intensity within the westward drifting black patch observed in Event 4B is very small compared to Events 4A and 4C. These three events are very close in time; Event $4 \mathrm{~A}$ occurs approximately 1 minute before, and Event 4C 10 seconds after Event 4B. The reason for the small relative decrease seems to be that the OI emission intensity decreases within the diffuse aurora during Event 4B, but does not decrease to a corresponding degree within the black patch. Therefore, the relative reduction in OI emissions within the black patch is only $9 \%$.

\subsubsection{Intensity measurements of pulsating events}

Figure 6.1 shows the mean intensity in ASK 1 and ASK3 of a vertical slice of width 10 pixels centred at xpixel $=50$ for two frames from Event $3 \mathrm{~A}$. The first frame is recorded during a pulsation maximum, and is plotted in red. The second frame is recorded $2.6 \mathrm{~s}$ later during the pulsation minimum seen in Figure 4.11, and is plotted in black. The drift speed of the black structures was undetectable at this time.

This is a clear case of total veiling, as previously described by Kimball and Hallinan (1998a). The pulsations add more to the intensity of the black structures than to the intensity of the surrounding diffuse aurora. At the pulsation maximum, the intensity level is almost identical across the whole field of view.

Figure 6.2 shows the same pulsation minimum in black, as well as slices from three subsequent frames recorded $0.5 \mathrm{~s}$ (blue), $1.0 \mathrm{~s}$ (green) and $1.5 \mathrm{~s}$ (red) later. At first, the intensity increase is primarily additive (blue line); however, when the pulsation approaches its maximum (green and red lines), the intensity levels within the black structures 'catch up' to the level of the diffuse aurora.

Figure 6.3 shows the mean intensity of a vertical slice (black line) through the middle of frame 4, Figure 4.21. The pulsation maximum (red line) occurs $0.9 \mathrm{~s}$ prior to the pulsation minimum. This event, Event $6 \mathrm{D}$, differs from Event $3 \mathrm{~A}$ in that the pulsations seem only to occur within the region of the black structures. While the pulsations observed during Event $3 \mathrm{~A}$ cover the whole frame of view, the pulsations 

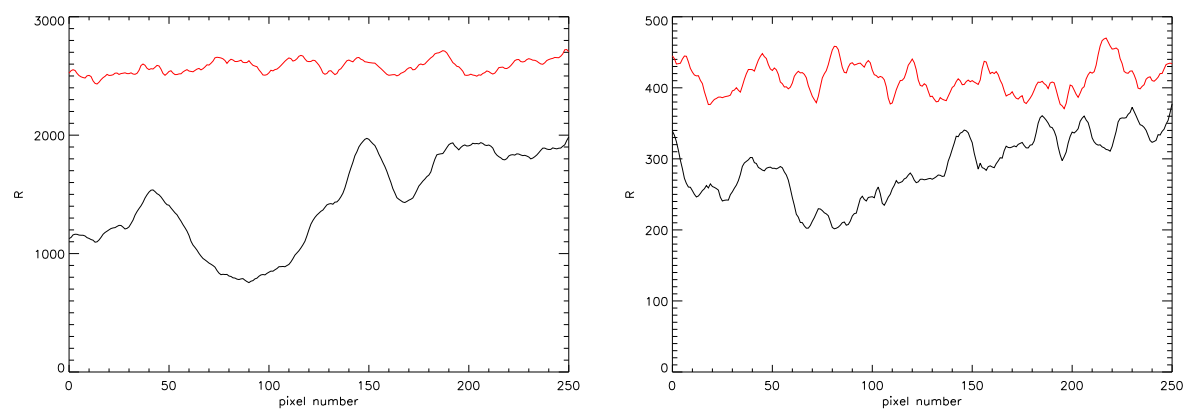

Figure 6.1: Event 3A. Intensity in ASK1 (left) and ASK3 (right) of vertical slice at xpixel=50. Red pulsation maximum at 20:24:30.219, black pulsation minimum at 20:24:32.844.

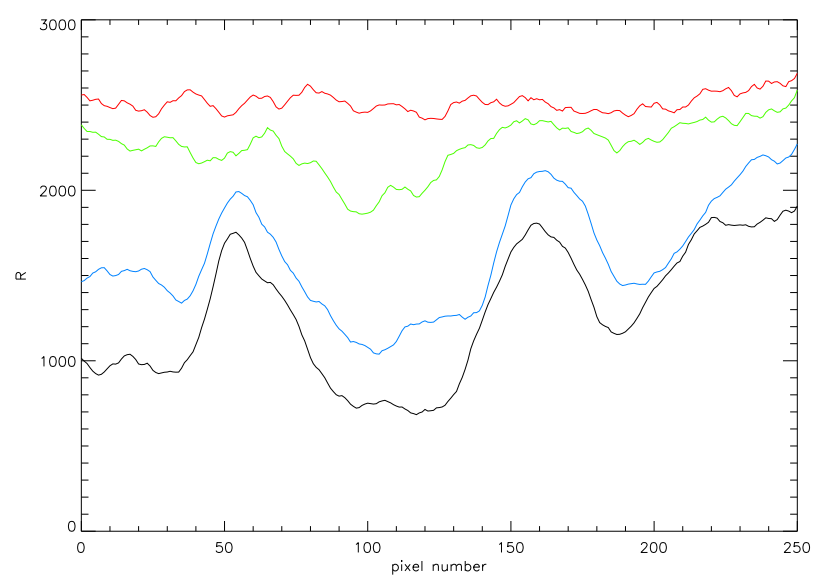

Figure 6.2: Event 3A. Intensity in ASK1 of vertical slice at xpixel=30. Black pulsation minimum at 20:24:32.844, red pulsation maximum at 20:24:34.344. 

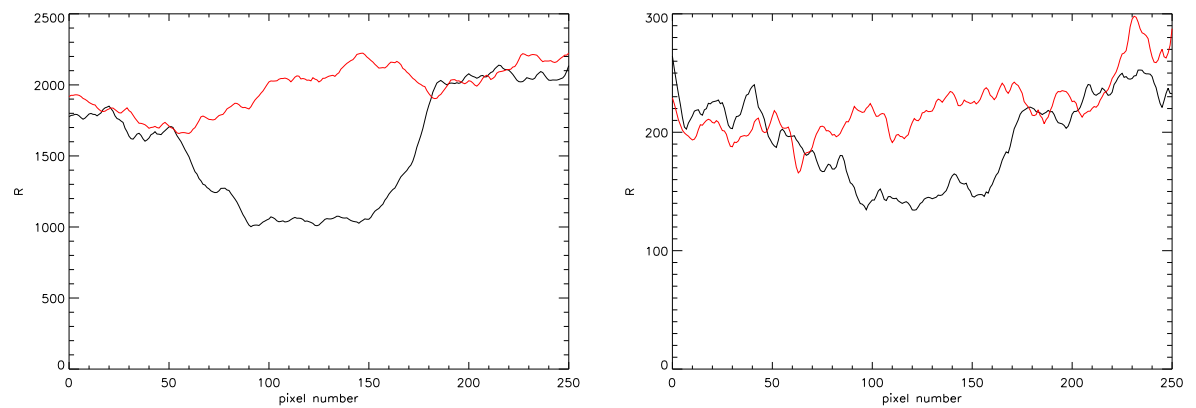

Figure 6.3: Event 6D. Intensity in ASK1 (left) and ASK3 (right) of vertical slice at xpixel=128. Red pulsation maximum at 23:06:00.800, black pulsation minimum at 23:06:01.700.

observed in Event 6D are localized to the black structures, although the black regions change location and shape between pulsations.

Another interesting observation is that the pulsation itself seems to constitute a sudden intensity burst or pulse, immediately followed by a sudden intensity depletion with the morphology of a typical black structure. The intensity of the bursts appears to be slightly higher than the intensity of the surrounding diffuse aurora.

These features can be seen quite clearly in the keogram, Figure 6.4. The pulsations are localized to the black structures. The intensity is higher immediately to the left of several of the dark regions observed in the keogram compared to the diffuse aurora.

\subsection{Intensity Ratios}

Figures 6.5, 6.6, 6.7 and 6.8 show simultaneous images from ASK1 and ASK3 together with the ratio of the images ASK3/ASK1. The black structures seen in the ASK1 and ASK3 images show up as white areas in the ratio plot. This means that the $\mathrm{OI} / \mathrm{N}_{2}$ ratio is larger within the dark regions than within the diffuse aurora, i.e. the $\mathrm{N}_{2}$ emissions decrease more than the OI emissions in the black regions.

This was found to be the case for all sixteen events, with the exception of Event 5A. As can be seen in Table 6.2, the relative reduction in intensity within the black vortex street is only $10 \%$ and $8 \%$ respectively, in the two measured emissions. Therefore, the difference in relative intensity reduction is not large enough to be significant for Event $5 \mathrm{~A}$.

The appearance of the OI/ $\mathrm{N}_{2}$ ratio images for the events in group III), which do not qualify as black aurora according to the definition, was analogous to the proper black auroral events; see Figures 5.5 and 6.9. The $\mathrm{N}_{2}$ emissions decrease more than the OI emissions in the dark regions situated adjacent to bright, discrete aurora that were observed during these two events.

Quantitative estimates of $\mathrm{OI} / \mathrm{N}_{2}$ emission intensity ratios are given in Table 6.3 for black structures and for the surrounding diffuse aurora (same time intervals as Ta- 


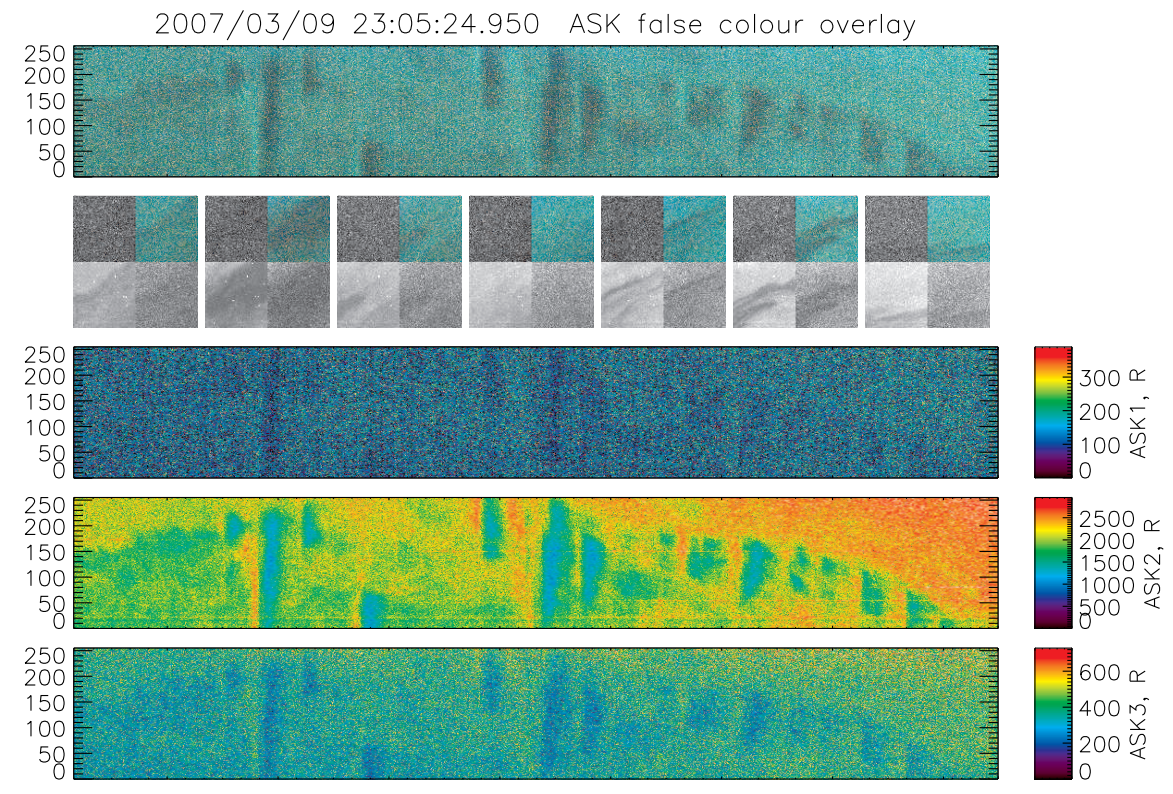

23:05:30 23:05:36 23:05:42 23:05:48 23:05:54 23:06:00 23:06:06 23:06:12

Figure 6.4: Keogram of Event 6D. Note that the $6730 \AA$ filter was fitted to the ASK2 camera during this event, although it is referred to as ASK1 throughout the text.
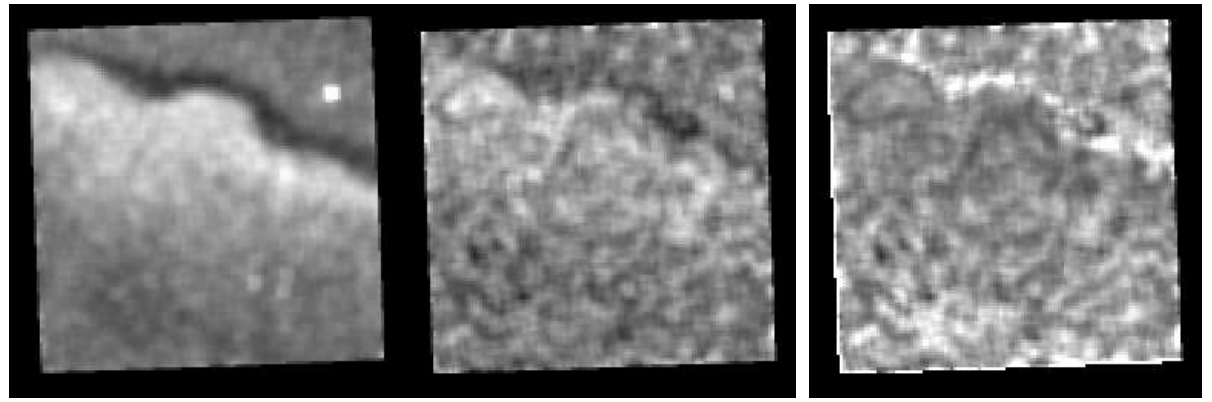

Figure 6.5: Event 2B. Simultaneous images ASK1, ASK3, ASK3/ASK1 intensity ratio. 


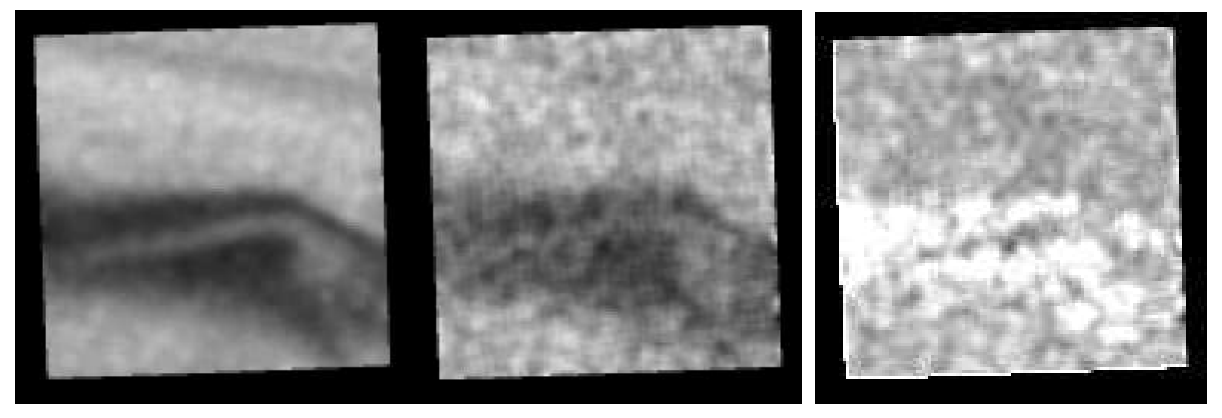

Figure 6.6: Event 3B. Simultaneous images ASK1, ASK3, ASK3/ASK1 intensity ratio.
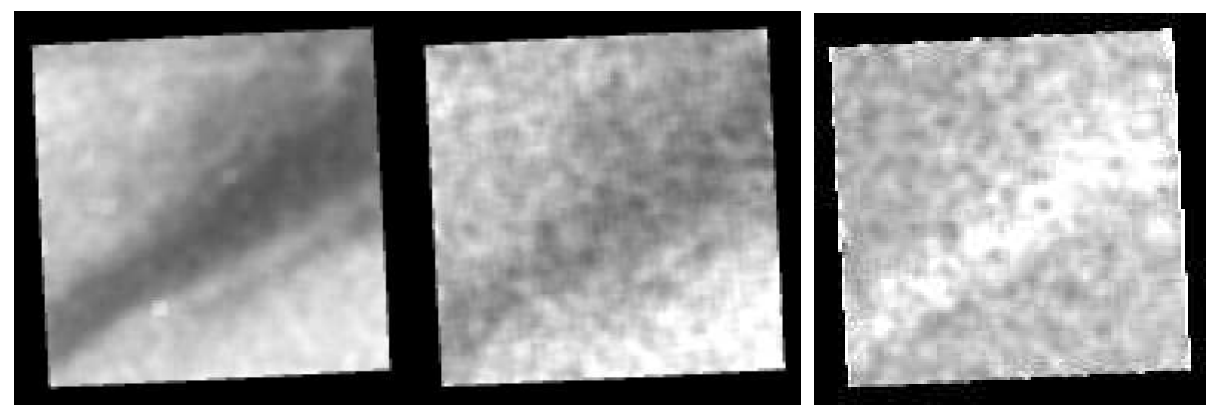

Figure 6.7: Event 6D. Simultaneous images ASK1, ASK3, ASK3/ASK1 intensity ratio.
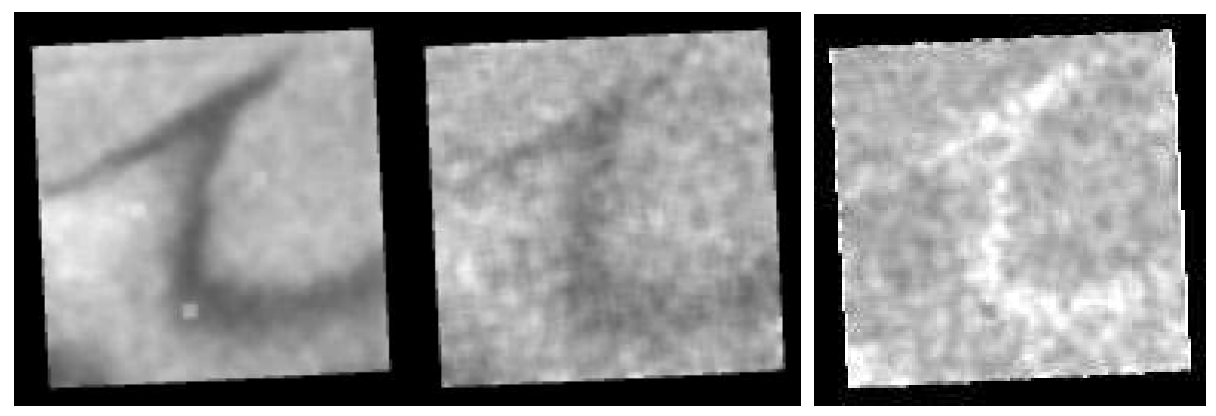

Figure 6.8: Event 6E. Simultaneous images ASK1, ASK3, ASK3/ASK1 intensity ratio. 

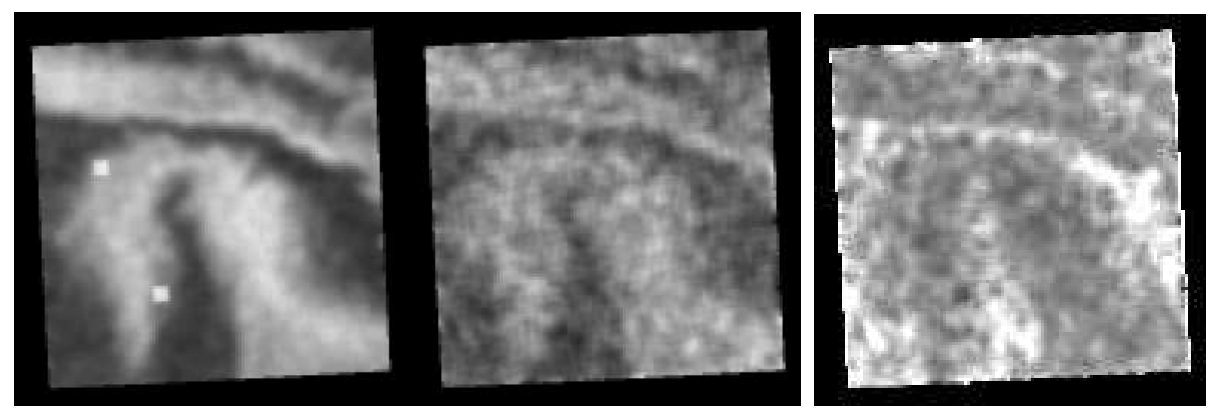

Figure 6.9: Event 6A. Simultaneous images ASK1, ASK3, ASK3/ASK1 intensity ratio.

ble 6.2). The OI/ $\mathrm{N}_{2}$ ratio is higher within the black aurora than the diffuse aurora for all the analysed events. The $\mathrm{OI} / \mathrm{N}_{2}$ ratio is $0.26-0.42$ for the black aurora, and 0.23 - 0.35 for the diffuse aurora. The average $\mathrm{OI} / \mathrm{N}_{2}$ ratio is determined to be 0.33 for the black structures, and 0.28 for the diffuse aurora.

Since $\mathrm{N}_{2}$ emissions are produced by high-energy electron precipitation and OI emissions are mainly caused by low-energy emissions, a greater depletion of $\mathrm{N}_{2}$ emissions than of OI emissions within dark structures indicates that high-energy electron precipitation decreases more than low-energy electron precipitation within these regions. This is consistent with a magnetospheric blocking mechanism which prevents high-energy electrons from entering the loss cone above regions of black aurora, in accordance with Peticolas et al. (2002).

\subsection{Estimated Energy of Precipitating Electrons}

The Southampton ion chemistry model performed numerical calculations of the energy of the precipitating electrons using the given $\mathrm{OI} / \mathrm{N}_{2}$ ratios, Table 6.3. Although only the simplest mono-energetic energy distribution was used, it was possible to determine that the energy of the precipitating energies is lower within the black structures than within the surrounding diffuse aurora for all the analysed events, which corroborates the results of the previous sections.

There appears to be no correlation between the drift speeds of the observed black structures and the energy of the precipitating electrons within the diffuse aurora. The drift speeds of the five black patches and arc segments included in the energy analysis range from $1.4-2 \mathrm{~km} / \mathrm{s}$. The three black structures with drift speeds of $2 \mathrm{~km} / \mathrm{s}$ are surrounded by diffuse aurora in which the precipitating electron energies are $8.3 \mathrm{keV}$, $3.9 \mathrm{keV}$ and $3.6 \mathrm{keV}$, respectively. The arc segment with a speed of $1.4 \mathrm{~km} / \mathrm{s}$ is surrounded by $7.9 \mathrm{keV}$ precipitating electrons.

These results are not in agreement with Blixt et al. (2005) who observed that the drift speeds of eight black patches increased with increasing energy of the precipitating electrons in the surrounding diffuse aurora. 


\begin{tabular}{|l|c|c|c|c|}
\hline & \multicolumn{2}{|c|}{ Black Aurora } & \multicolumn{2}{c|}{ Diffuse Aurora } \\
Event & OI/ $\mathrm{N}_{2}$ ratio & Energy, $\mathrm{keV}$ & $\mathrm{OI}_{2}$ ratio & Energy, keV \\
\hline \hline & & & & \\
2B & 0.35 & 4.1 & 0.28 & 6.3 \\
2C & 0.34 & 4.3 & 0.29 & 5.8 \\
2D & 0.31 & 4.9 & 0.26 & 7.2 \\
& & & & \\
3B & 0.33 & 3.1 & 0.25 & 3.6 \\
& & & & \\
4A & 0.26 & 7.3 & 0.23 & 9.3 \\
4B & 0.32 & 5.1 & 0.24 & 8.3 \\
4C & 0.29 & 6.0 & 0.25 & 7.9 \\
& & & & \\
5A & 0.33 & 4.0 & 0.32 & 4.2 \\
& & & & \\
6B & 0.34 & 3.1 & 0.30 & 4.0 \\
6C & 0.36 & 2.9 & 0.31 & 3.9 \\
6E & 0.42 & 2.1 & 0.35 & 3.0 \\
& & & & \\
\hline
\end{tabular}

Table 6.3: Estimated energy of precipitating electrons within black aurora and diffuse aurora.

\subsection{Comparison of Optical and Radar Data}

Figure 6.10 depicts EISCAT electron density measurements during a 20-minute interval which includes Event $1 \mathrm{~A}$ at 22:37 - 22:44. The optical observations show that the aurora is pulsating with a frequency of $0.1 \mathrm{~Hz}$, see Figure 4.3. Black arc segments and patches are visible during the pulsatation minimums for approximately $5 \mathrm{~s}$ at a time before becoming obscured by the increasing intensity of the next pulsation.

No evidence of the pulsations or the black structures is visible in the electron density image. This may be due to the low time resolution ( $4 \mathrm{~s}$ ) of the radar data, as well as the relatively large diameter of the radar beam compared to the width of the black structures. However, some slower pulsations with a period of about 1 minute seem to occur simultaneously with the faster pulsations; this feature can also be seen in Figure 4.3, and is clearest at around 22:38.

The auroral emissions originate in the $\mathrm{E}$ layer which is indicative of high-energy electron precipitation. There is no evidence of dual layers of aurora with separate emission altitudes and energy distributions, as suggested by Trondsen and Cogger (1997), unless the top layer is at a higher altitude than $200 \mathrm{~km}$. This is unlikely, since the morphology of the aurora is the same in ASK1 and ASK3.

The diameter of the radar beam is approximately $1 \mathrm{~km}$ at $105 \mathrm{~km}$ altitude. This means that when the narrow black arc observed in Event 2B crosses the radar beam, it does not fill the whole radar beam circle. Therefore no decrease in intensity is observed until 20:51:00 - 20:51:15, when the black arc widens and almost completely fills the 


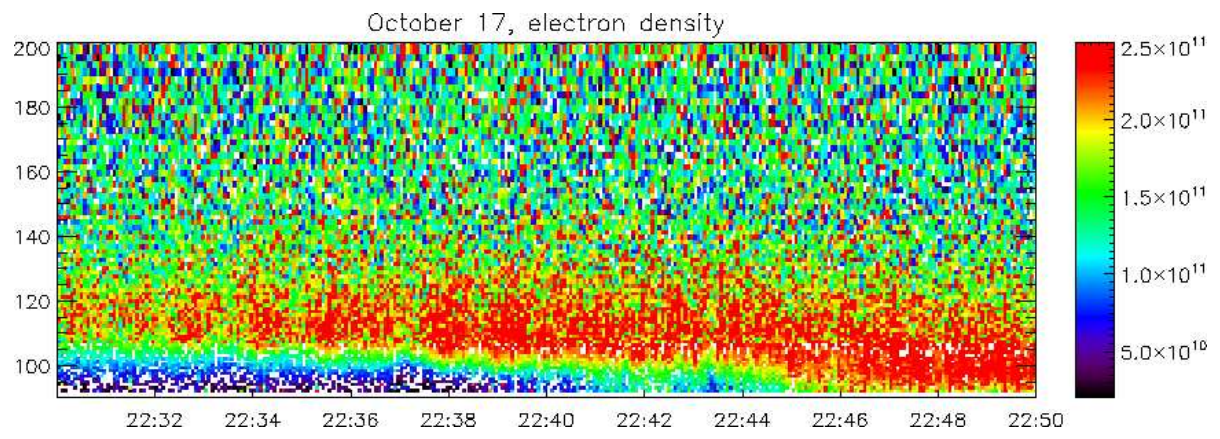

Figure 6.10: Event 1A. Radar image of electron density. Altitude, km (left), particle density, $\mathrm{m}^{-3}$ (right).

radar beam circle for ca $15 \mathrm{~s}$, see Figure 6.11, top plot. It is possible to discern a slight electron density depletion, see Figure 6.11, middle and bottom plots.

Similarly, during Event 2C, the only large intensity decrease occurs from 20:52:31 - 20:52:36, when a black structure briefly fills the radar beam circle for $5 \mathrm{~s}$. This event exhibits an observable electron density depletion. The black arc observed in Event 2D is wide enough to fill the radar beam circle for approximately $35 \mathrm{~s}$, from 20:55:05 20:55:40. The in-beam intensity decreases simultaneously with the electron density.

The broad intensity peak which occurs immmediately prior to the intensity depletion of Event 2D is due to the bright arc situated equatorward of the black arc seen in Figure 4.9. The electron density image shows that the southward edge of this bright arc, which is quite diffuse, is produced by lower energy precipitation followed by highenergy precipitation, while the sharp northward boundary appears to be caused by electrons of intermediate energy. 

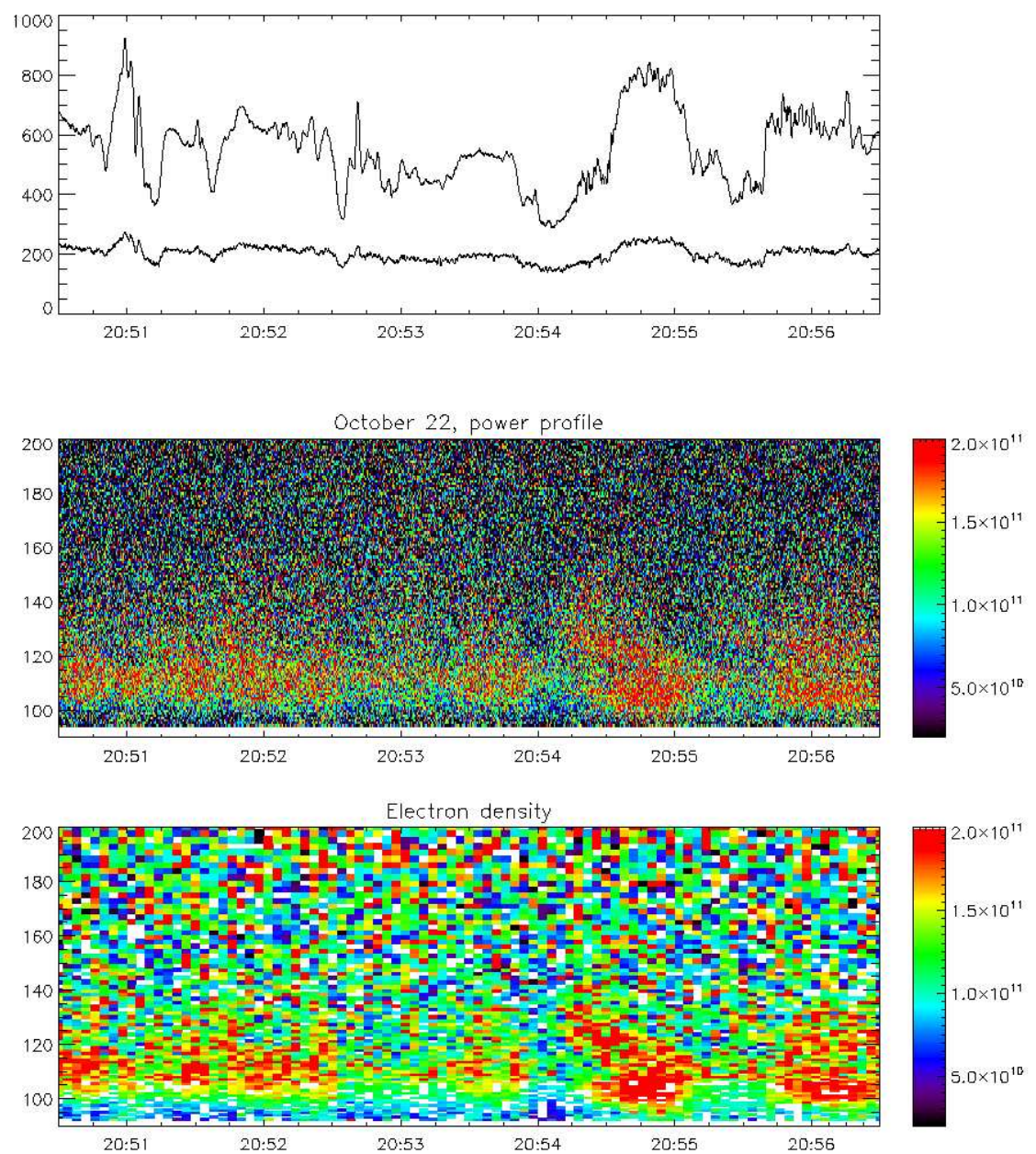

Figure 6.11: Events 2B, 2C, 2D. From top: mean in-beam intensity, Rayleighs, in ASK1, ASK3; radar images of power profile and electron density, with altitude, km (left), particle density, $\mathrm{m}^{-3}$ (right). 


\section{Chapter 7}

\section{Conclusions}

Sixteen black auroral events detected by the ASK instrument have been analysed. The black structures were found predominantly in the midnight sector diffuse aurora on the equatorward edge of the auroral oval at Troms $\emptyset$, Norway. No black aurora was found in the Svalbard data.

The black structures are not devoid of optical emissions. The average relative intensity reductions within eleven dark regions compared to the surrounding diffuse aurora are $35 \%$ for $\mathrm{N}_{2}$ emissions and $23 \%$ for OI emissions. Two regions, shown in Events $2 \mathrm{~A}$ and $6 \mathrm{~A}$, which constitute the dark space adjacent to discrete bright arcs are included for comparison with the proper black auroral events. The results are the same: $\mathrm{N}_{2}$ emissions decrease more than OI emissions in the dark regions. The only exception is the black vortex street seen in Event 5A. Although the relative decrease in $\mathrm{N}_{2}$ emission intensity is greater than the relative decrease in OI emission intensity, $10 \%$ and $8 \%$ respectively, the difference is too small to be significant.

Therefore, the $\mathrm{N}_{2}$ emissions caused by high-energy electron precipitation decrease significantly more than the OI emissions produced mainly by low-energy electron precipitation within fifteen of the observed dark structures. This result is confirmed by the electron energy estimations performed using the Southampton ion chemistry model; the energies of the precipitating electrons are lower within black aurora than within diffuse aurora for the eleven studied events.

The results are consistent with a magnetospheric blocking mechanism which prevents high-energy electrons from entering the loss cone above regions of black aurora, thereby giving rise to a partial double loss cone distribution. The case of total veiling, Event $3 \mathrm{~A}$, also supports this theory; the black structures attain the same intensity as the diffuse aurora during the pulsation maximum, which may be connected to the strong pitch angle diffusion limit.

Peticolas et al. (2002) observed that the partial double loss cone distribution was dominant in the central plasma sheet region of the auroral oval (Troms $\varnothing$ latitudes) on the six nights without black aurora. This may give an indication as to why the dark spaces between discrete bright aurora observed in Events 2A and 6A also exhibit significantly larger depletions in high-energy than low-energy electron precipitation. The limiting case observed in Event 2D shows that there is no clear-cut way of differentiat- 
ing which structures actually constitute proper black aurora.

The EISCAT electron density data for Events $2 \mathrm{~B}, 2 \mathrm{C}$ and 2D show a significant electron density depletion occurring simultaneously with the in-beam intensity decrease of Event 2D. Events 2B and 2C also exhibit possible electron density depletions, however, these results are not as clear. This could be due to the lower time resolution of the radar data compared to the optical data.

Future work The ASK data contain many more black auroral events than the sixteen that were analysed in this study. It would be interesting (and time consuming) to perform a thorough search of the ASK data from Troms $\varnothing$ and Svalbard, catalogue the events, and perform statistical analyses.

The comparison of a large number of events constituting dark regions adjacent to discrete, bright aurora with proper black auroral events might provide further information about the composition and origin of black aurora. 


\section{Bibliography}

[Blixt and Kosch, 2004] Blixt, E. M., and Kosch, M. J., Coordinated optical and EISCAT observations of black aurora, Geophys. Res. Lett., 31, L06813, doi:10.1029/2003GL019244, 2004.

[Blixt et al., 2005] Blixt, E. M., Kosch, M. J., and Semeter, J., Relative drift between black aurora and the ionospheric plasma, Ann. Geophys., 23, 1611-1621, 2005.

[Dahlgren, 2008] Dahlgren, Hanna, Fine-scale morphology and spectral characteristics of active aurora, Licentiate Thesis in Physical Electrotechnology, Space and Plasma Physics, School of Electrical Engineering, KTH, Stockholm, Sweden, 2008.

[Dahlgren et al., 2008] Dahlgren, H., Ivchenko, N., Sullivan, J., Lanchester, B. S., Marklund, G., and Whiter, D., Morphology and dynamics of aurora at fine scale: first results from the ASK instrument, Ann. Geophys., 26, 1041-1048, 2008.

[Davis, 1978] Davis, T. N., Observed microstructure of auroral forms, J. Geomagn. Geoelectr., 30, 371-380, 1978.

[Gustavsson et al., 2008] Gustavsson, B., Kosch, M. J., Senior, A., Kavanagh, A. J., Brändström, B. U. E., and Blixt, E. M., Combined EISCAT radar and optical multispectral and tomographic observations of black aurora, J. Geophys. Res., 113, A06308, doi:10.1029/2007JA012999, 2008.

[Johnson and Chang, 1995] Johnson, Jay R., and Chang, Tom, Nonlinear vortex structures with diverging electric fields and their relation to the black aurora, Geophys. Res. Lett., 22(12), 1481-1484, 1995.

[Keskinen and Ganguli, 1996] Keskinen, M. J., and Ganguli, G., Simulation of dynamics and structure in the black aurora, J. Geophys. Res., 101, A12, 26995-26999, December 1, 1996.

[Kimball and Hallinan, 1998a] Kimball, J., and Hallinan, T. J., Observations of black auroral patches and of their relationship to other types of aurora, J. Geophys. Res., 103, A7, 14671-14682, July 1, 1998.

[Kimball and Hallinan, 1998b] Kimball, J., and Hallinan, T. J., A morphological study of black vortex streets, J. Geophys. Res., 103, A7, 14683-14695, July 1, 1998. 
[Kivelson and Russell, 1995] Kivelson, M. G., and Russell, C. T., Introduction to Space Physics. Introduction to Space Physics, Edited by Margaret G. Kivelson and Christopher T. Russell, pp. 568. ISBN 0521451043. Cambridge, UK: Cambridge University Press, April 1995.

[Lanchester et al., 2001] Lanchester, B. S., Rees, M. H., Lummerzheim, D., Otto, A., Sedgemore-Schulthess, K. J. F., Zhu, H., and McCrea, I. W., Ohmic heating as evidence for strong field-aligned currents in filamentary aurora, J. Geophys. Res., 106, A2, 1785-1794, February 1, 2001.

[Marklund et al., 1994] Marklund, Göran, Blomberg, Lars, Fälthammar, Carl-Gunne, and Lindqvist, Per-Arne, On intense diverging electric fields associated with black aurora, Geophys. Res. Lett., 21(17), 1859-1862, 1994.

[Marklund et al., 1997] Marklund, Göran, Karlsson, Tomas, and Clemmons, James, On low-altitude particle acceleration and intense electric fields and their relationship to black aurora, J. Geophys. Res., 102, A8,17509-17522, August 1, 1997.

[Marklund et al., 2001] Marklund, G. T., Ivchenko, I., Karlsson, T., Fazakerley, A., Dunlop, M., Lindqvist, P.-A., Buchert, S., Owen, C., Taylor, M., Vaivalds, A., Carter, P., André, M., and Balogh, A., Temporal evolution of the electric field accelerating electrons away from the auroral ionosphere, Nature, 414, 724-727, 2001.

[Marklund et al., 2004] Marklund, G. T., Karlsson, T., Figueiredo, S., Johansson, T., Lindqvist, P.-A., André, M., Buchert, S., Kistler, L. M., and Fazakerley, A., Characteristics of quasi-static potential structures observed in the auroral return current region by Cluster, Nonlinear Processes in Geophysics (2004) 11: 709-720, 2004.

[Oguti, 1975] Oguti, T., Metamorphoses of aurora, Mem. Natl. Inst. Polar Res. Spec. Issue Jpn., no. 12, 101pp., 1975.

[Parsons and Thomas, 1973] Parsons, N. R., and Thomas, I. L., Spatially forbidden regions in the aurora, Can. J. Phys., 51, 1377-1378, 1973.

[Peticolas et al., 2002] Peticolas, L. M., Hallinan, T. J., Stenbaek-Nielsen, H. C., Bonnell, J. W., and Carlson, C. W., A study of black aurora from aircraft-based optical observations and plasma measurements on FAST, J. Geophys. Res., 107, A8, 1217, 10.1029/2001JA900157, 2002.

[Royrvik, 1976] Royrvik, O., Pulsating aurora: Local and global morphology, Ph.D. thesis, Univ. of Alaska, Fairbanks, 1976.

[Sakanoi et al., 2008] Sakanoi, T., Obuchi, Y., Okano, S., Yamazaki, A., Asamura, K., Kasaba, Y., Hirahara, M., Ebihara, Y., and Seki, K., Fine-scale dynamics of black aurora obtained from simultaneous image-particle observation data by the Reimei satellite, 37th COSPAR Scientific Assembly, Held 13-20 July 2008, in Montreal, Canada, p. 2710, 2008. 
[Shukla et al., 1995] Shukla, P. K., Birk, G. T., and Bingham, R., Vortex streets driven by sheared flow and applications to black aurora, Geophys. Res. Lett., 22(6), 671$674,1995$.

[Trondsen and Cogger, 1997] Trondsen, T. S., and Cogger, L. L., High-resolution television observations of black aurora, J. Geophys. Res., 102, A1, 363-378, January 1, 1997.

[Trondsen, 1998] Trondsen, Trond S., High Spatial and Temporal Resolution Auroral Imaging, A dissertation for the degree of Doctor Scientiarum, Department of Physics, Faculty of Science, University of Troms $\varnothing$, Norway, 1998.

[Weiss et al., 1992] Weiss, L. A., Reiff, P. H., Hilmer, R. V., Winningham, J. D., and $\mathrm{Lu}, \mathrm{G}$., Mapping the Auroral Oval into the Magnetotail Using Dynamics Explorer Plasma Data, J. Geomag. Geoelectr., 44, 1121-1144, 1992. 


\section{IDL Routines}

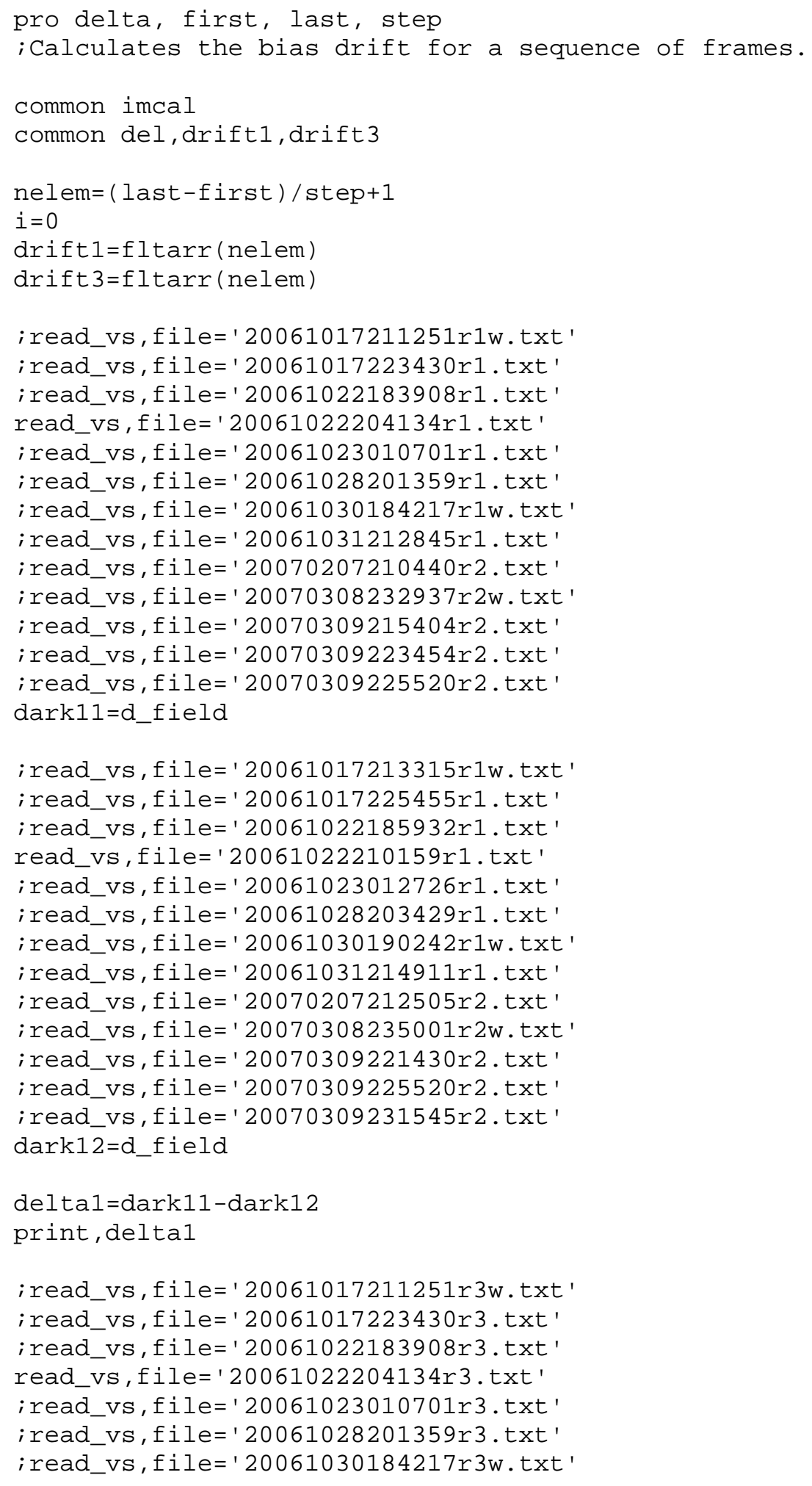




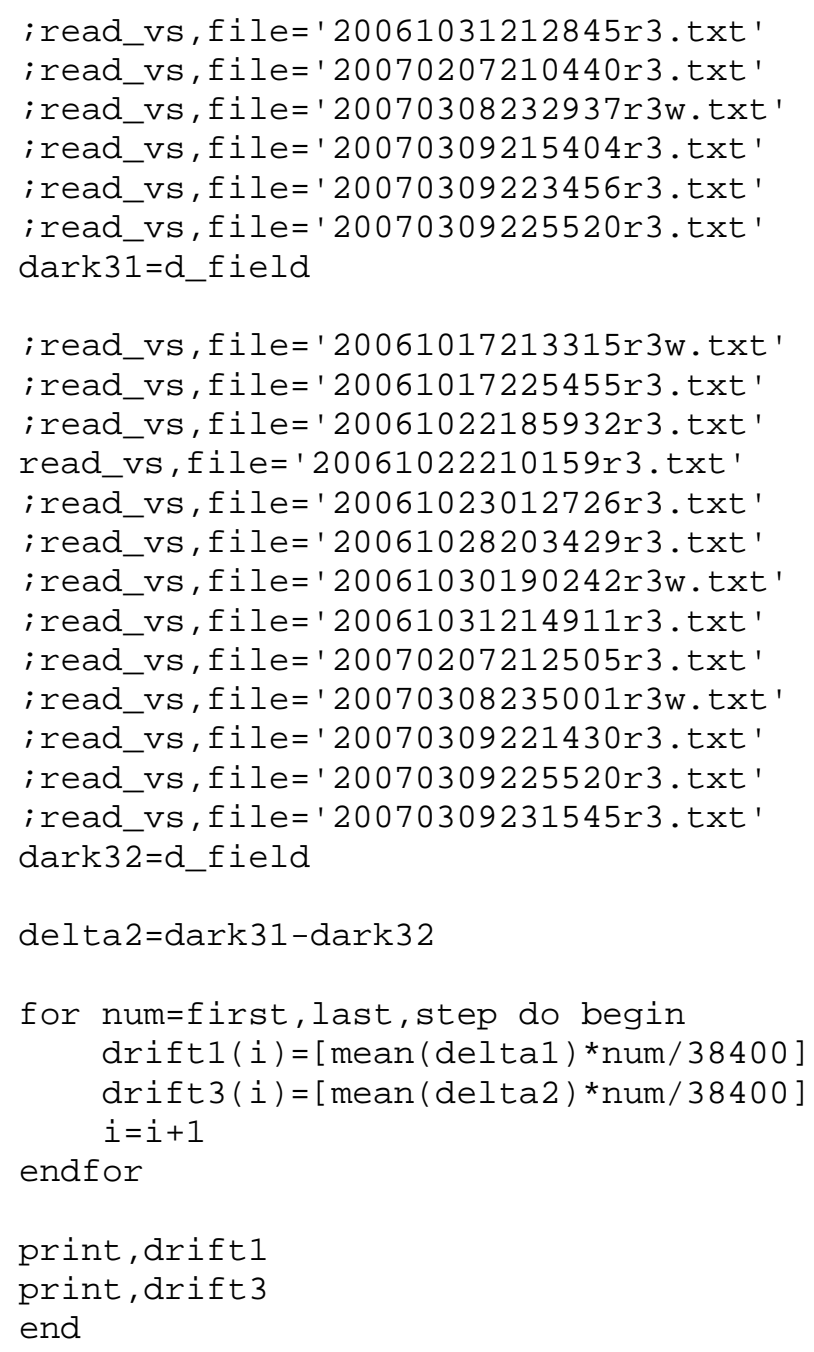


pro align_pic

; Rotates and aligns images so that north is pointing upwards.

common share, iout 1, jout1, iout 2, jout 2, iout3, jout3

; read_vs,file=['20061017223430r1.txt ' , '20061017223430r2.txt ' , '20061017223430r3.t $\left.x t^{\prime}\right]$

; read_vs,file $=[$ '20061022183908r1.txt ' , '20061022183908r2.txt ' , '20061022183908r3.t $\left.x t^{\prime}\right]$

; read_vs, file $=[$ ' 20061022204134r1.txt ' , '20061022204134r2.txt ' , '20061022204134r3.t $\left.x \mathrm{t}^{\prime}\right]$

;read_vs,file $=[$ '20061028201359r1.txt ' , '20061028201359r2.txt ' , '20061028201359r3.t $\left.x t^{\prime}\right]$

read_vs,file $=[$ '20061031212845r1.txt ', '20061031212845r2.txt ', '20061031212845r3.tx t' ]

; read_vs, file $=[$ '20070207210440r1.txt ' , '20070207210440r2.txt ' , '20070207210440r3.t $\left.x t^{\prime}\right]$

; read_vs,file $=[$ ' 20070309215404r1.txt ' , '20070309215404r2.txt ' , '20070309215404r3.t $\left.x t^{\prime}\right]$

; read_vs,file $=[$ '20070309223456r1.txt ' , '20070309223454r2.txt ' , '20070309223456r3.t $\left.x t^{\prime}\right]$

; read_vs, file=['20070309225520r1.txt ' , '20070309225520r2.txt ' , '20070309225520r3.t $\left.x t^{\prime}\right]$

v_select, $\odot$

get_cnv, cnv, scale

remap, fltarr $(256,256), 0.0, \odot . \odot, \odot .031,128 * 2,128 * 2$, iout1, jout1, cnv, /tromso

$v \_$select, 1

get_cnv, cnv, scale

remap, fltarr $(256,256), 0.0,0.0,0.031,128 * 2,128 * 2$, iout2, jout2, cnv, /tromso

v_select, 2

get_cnv, cnv, scale

remap, fltarr $(256,256), \odot . \odot, \odot . \odot, 0.031,128 * 2,128 * 2$, iout3, jout3, cnv, /tromso 
pro int_time, first, last, step, width, xvalue, yvalue, background, name=name ; Calculates the mean intensity over time within a square region of ; width 'width' centred at xvalue, yvalue

common vs

common del

nelem $=($ last - first $) /$ step +1

$i=0$

int1=fltarr (nelem)

int3=fltarr (nelem)

for num=first, last, step do begin

for $j=0,2$ do begin

$v \_$select, $j$, /quiet

read_v, num, data;, /raw

if $j$ eq $\odot$ then begin

data $=$ data - background $(\Theta)+\operatorname{drift} 1$ (i)

cnvfactor $=0.52$

endif

if $j$ eq 1 then begin

; data $=$ data - background (1)+drift1(i)

endif cnvfactor $=0.4$

if $j$ eq 2 then begin

data $=$ data - background (2) + drift3 $(i)$

cnvfactor $=0.90$

endif

; cnvfactor $=1.2$

; if $\operatorname{dimx}(v s e l)$ eq 512 then data=bin(data, $[2,2])$

calfac $=$ cnvfactor $/ v$ res (vsel)

data $=$ data*calfac

; data $=$ data* 4

square $=$ data $(x v a l u e-$ width $/ 2: x$ value - width $/ 2+$ width -1 , yvalue - width $/ 2:$ yvalue -

width/2+width-1)

square=median ( square, 3 )

aver=mean (square)

if $j$ eq $\odot$ then int $1(i)=$ aver

if $j$ eq 1 then int $1(i)=$ aver

if $j$ eq 2 then int3(i)=aver

endfor

$i=i+1$

endfor

; int1=leefilt (int 1,3 )

; int $3=$ leefilt (int 3,3$)$

print, int 1

print, int3

set_plot, 'ps' 


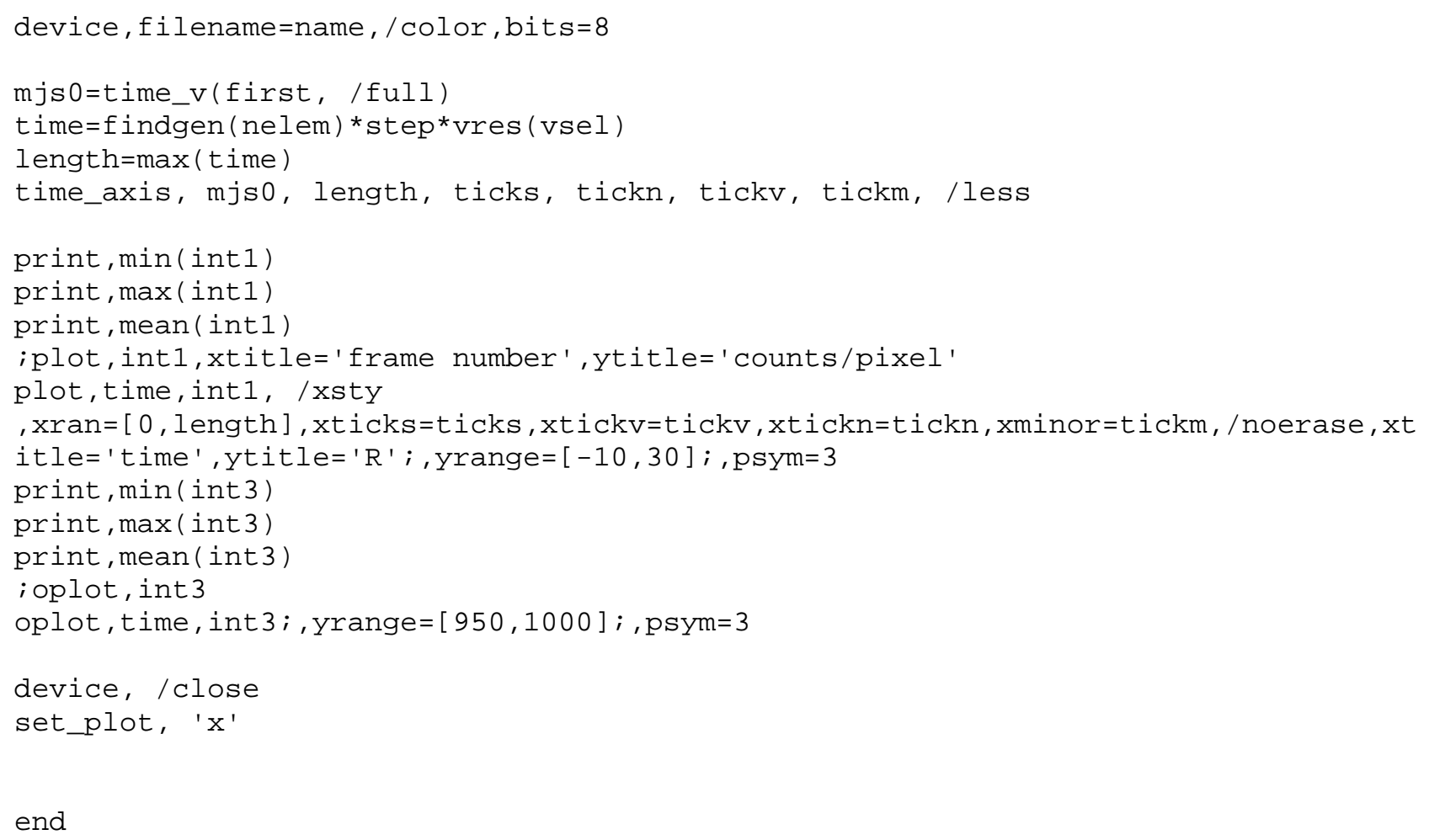

end 
pro int_radar, first, last, step, background, name=name ; Calculates the mean in-beam intensity over time

common vs

common del

nelem $=($ last - first $) /$ step +1

$i=0$

int1=fltarr (nelem)

int3=fltarr(nelem)

$\mathrm{nx}=\operatorname{dimx}(\mathrm{vsel})$

ny=dimy (vsel)

get_cnv, cnv, scale

$\mathrm{r}=0.4 / \mathrm{scale}$

rad_az $=185.02 *$ !dtor

rad_el $=77.5^{*}$ ! dtor

conv_xy_ae, x,y,rad_az,rad_el,cnv, /back

roundmask, nx, ny, $x, y, r$, mask, dummy

for num=first, last, step do begin

for $j=0,2$ do begin

v_select, j, /quiet

read_v, num, data

if $j$ eq $\odot$ then begin

data $=$ data - background $(0)+\operatorname{drift} 1$ (i)

endif

cnvfactor $=0.52$

if $j$ eq 2 then begin

data=data-background(2) +drift3(i)

endif

cnvfactor $=\odot .90$

calfac $=$ cnvfactor $/$ vres (vsel)

data $=$ data ${ }^{*}$ calfac

area $=$ data $($ mask $)$

circav=mean (data (mask))

if $j$ eq $\odot$ then int1(i)=circav

if $j$ eq 2 then int3(i)=circav

endfor

$i=i+1$

endfor

int1=leefilt (int1, 3)

int $3=$ leefilt $($ int 3,3$)$

print, int 1

print, int3

set_plot, 'ps'

device, filename=name, /color , bits $=8$

mjs $\theta=$ time_v(first, /full) 


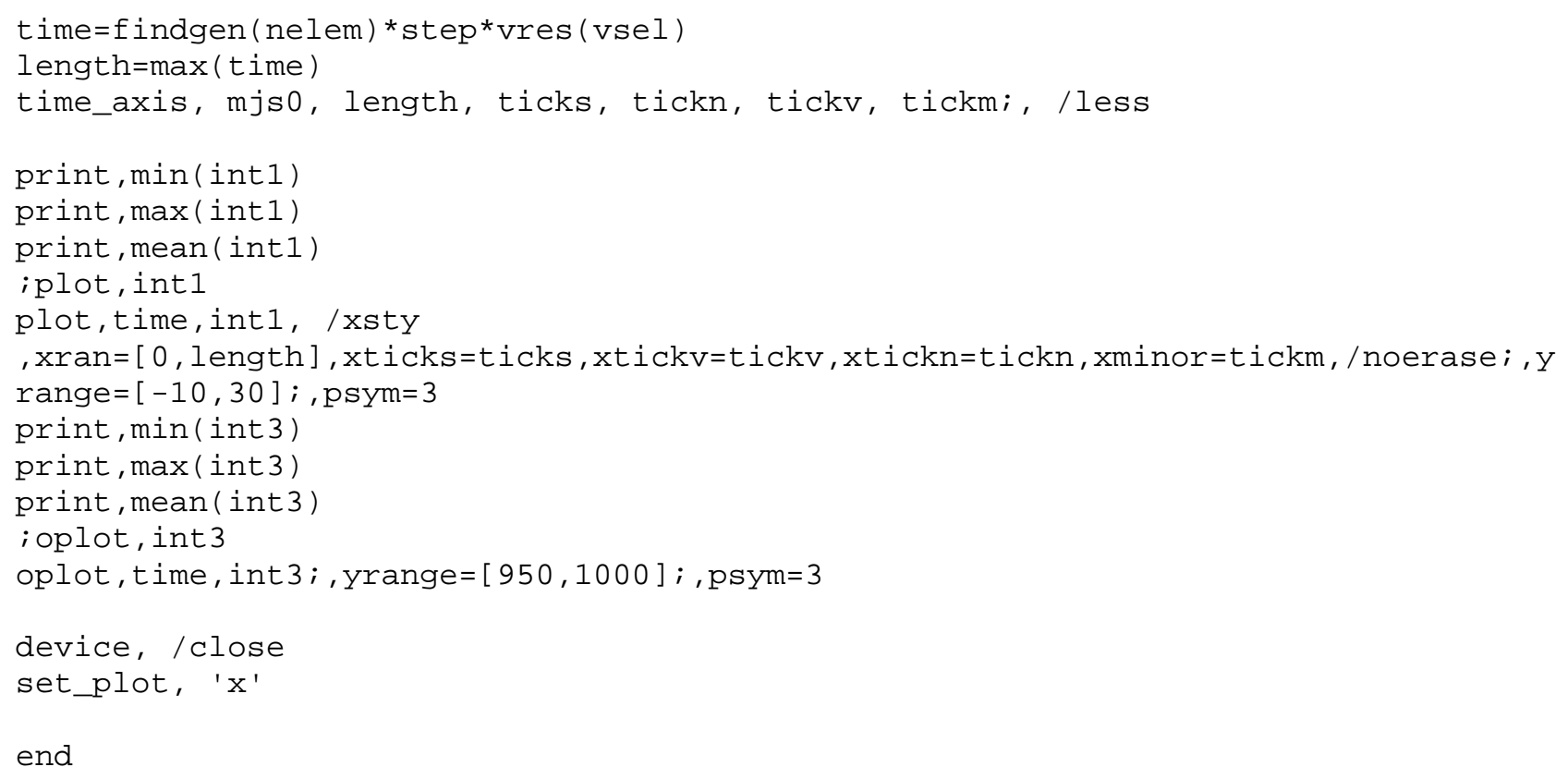


pro mean_int, first, last, step, background, psfile=psfile ; Calculates the intensity within regions of interest (dark, ; diffuse) for ASK1 and ASK3 for a sequence of frames

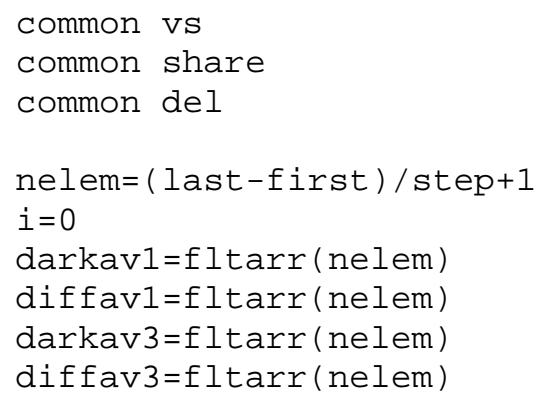

for num=first, last, step do begin

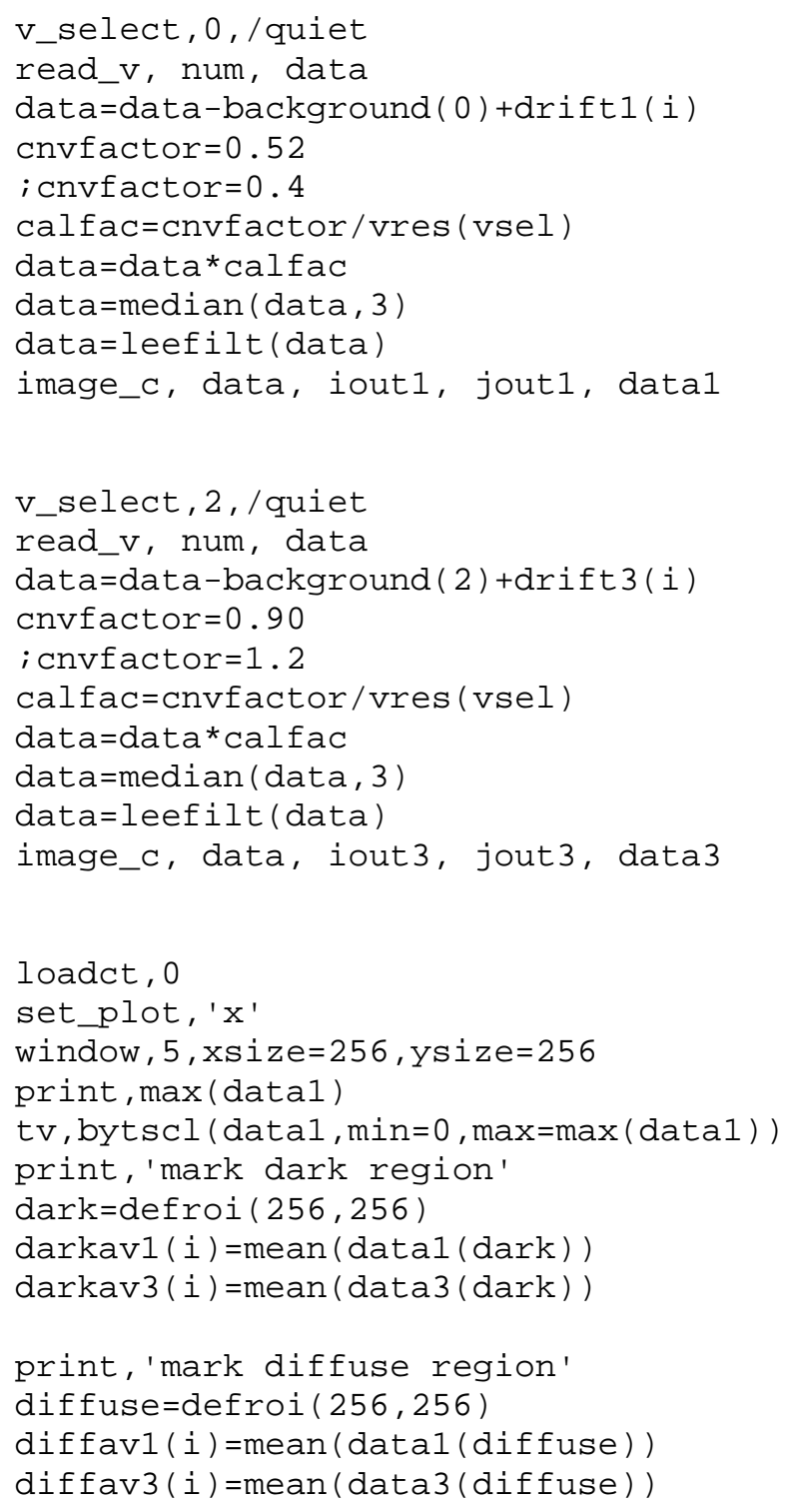




$$
i=i+1
$$

endfor

print, darkav1 print, diffav1 print, darkav3 print, diffav3

set_plot, 'ps'

device, filename=psfile, /color, bits=8

mjs $\theta=$ time_v(first, /full)

time $=$ findgen $($ nelem $){ }^{*}$ step $^{*}$ vres (vsel)

length $=\max (\mathrm{time})$

time_axis, mjs@, length, ticks, tickn, tickv, tickm, /less

print, $\min ($ darkav1)

print, $\max$ (darkav1)

print, mean (darkav1)

print, min(darkav3)

print, $\max ($ darkav3)

print, mean (darkav3)

plot, time, diffav1, /xsty

, $x r a n=[\Theta$, length] $, x t i c k s=t i c k s, x t i c k v=t i c k v, x t i c k n=t i c k n, x m i n o r=t i c k m, /$ noerase $;, y$ range $=[-10,30] ;$, psym $=3$

print, min(diffav1)

print, $\max$ (diffav1)

print, mean (diffav1)

print, $\min ($ diffav3)

print, $\max$ (diffav3)

print, mean (diffav3)

oplot, time, darkav1; , yrange $=[950,1000] ;$, psym $=3$

device, /close

set_plot, ' $x$ '

end 


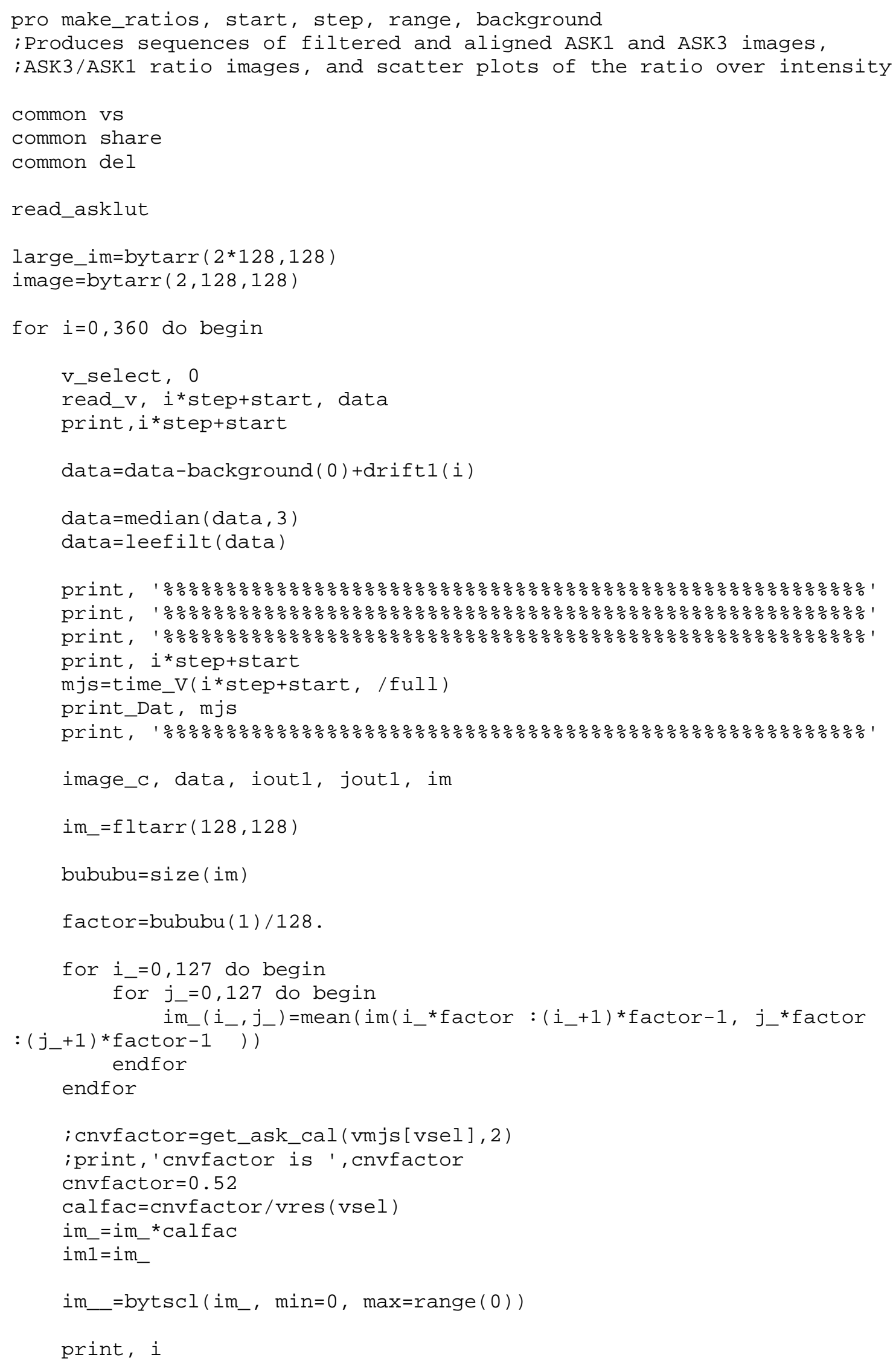




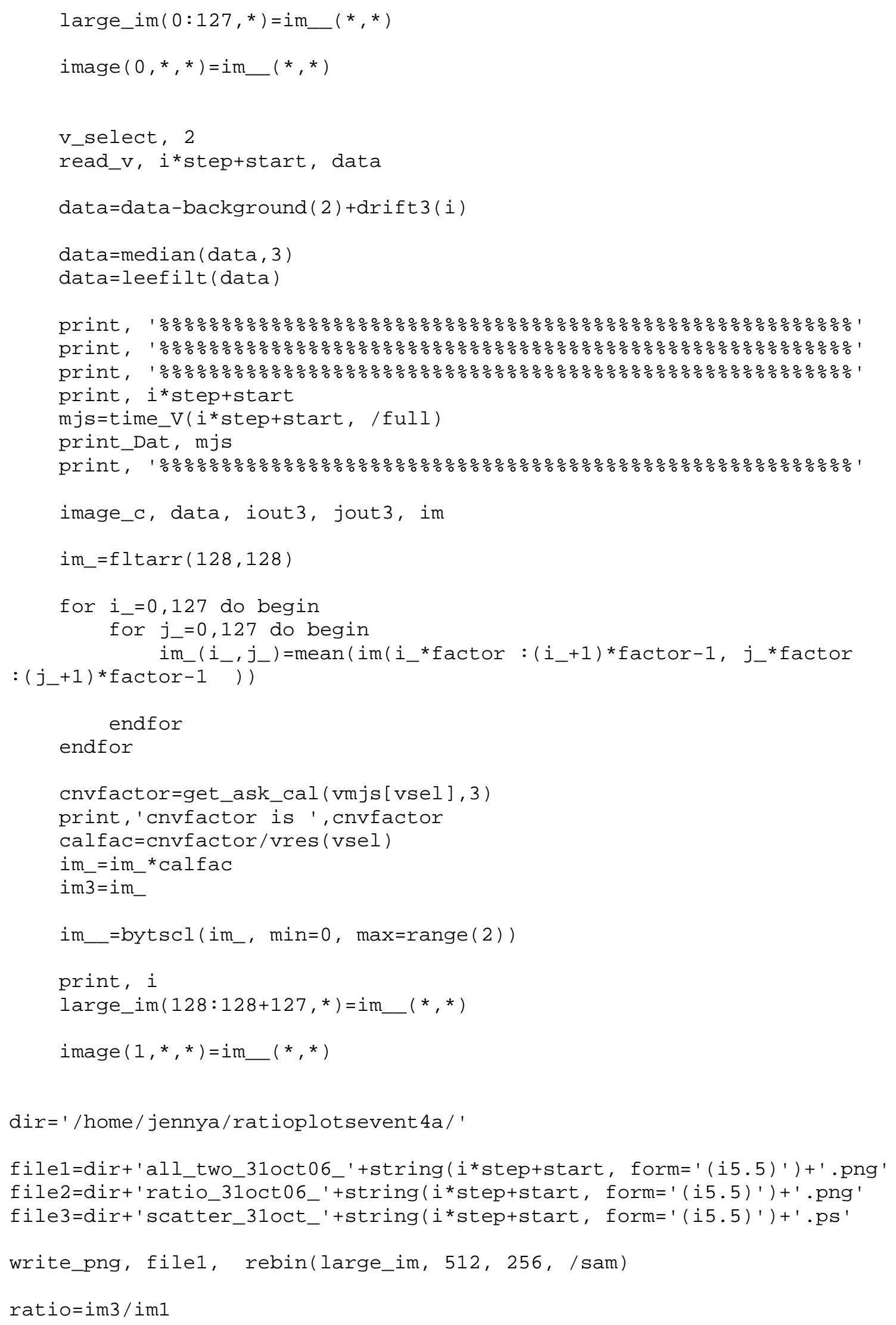


loadct, 39

set_plot, 'ps'

device, file=file3

plot, im1, ratio, psym $=3$, $x$ range $=[0,3000], \operatorname{yran}=[0.0,0.8]$, /ysty

; oplot, ratio(diffuse), color $=240$

device, /close

set_plot, ' $x$ '

; Iratio=rebin(ratio, 256, 256, /sam)

write_png, file2, bytscl(ratio, $\min =0, \max =0.36$ )

endfor

stop

end 
XR-EE-SPP 2009:002

www.kth.se 